\title{
Associated Legendre Functions and Spherical Harmonics of Fractional Degree and Order
}

\author{
Robert S. Maier ${ }^{1}$
}

Received: 25 February 2017 / Accepted: 30 August 2017 / Published online: 13 November 2017

(C) The Author(s) 2017. This article is an open access publication

\begin{abstract}
Trigonometric formulas are derived for certain families of associated Legendre functions of fractional degree and order, for use in approximation theory. These functions are algebraic, and when viewed as Gauss hypergeometric functions, belong to types classified by Schwarz, with dihedral, tetrahedral, or octahedral monodromy. The dihedral Legendre functions are expressed in terms of Jacobi polynomials. For the last two monodromy types, an underlying 'octahedral' polynomial, indexed by the degree and order and having a nonclassical kind of orthogonality, is identified, and recurrences for it are worked out. It is a (generalized) Heun polynomial, not a hypergeometric one. For each of these families of algebraic associated Legendre functions, a representation of the rank- 2 Lie algebra $\mathfrak{s o}(5, \mathbb{C})$ is generated by the ladder operators that shift the degree and order of the corresponding solid harmonics. All such representations of $\mathfrak{s o}(5, \mathbb{C})$ are shown to have a common value for each of its two Casimir invariants. The Dirac singleton representations of $\mathfrak{s o}(3,2)$ are included.
\end{abstract}

Keywords Associated Legendre function · Algebraic function · Spherical harmonic · Solid harmonic $\cdot$ Jacobi polynomial $\cdot$ Heun polynomial $\cdot$ Ladder operator

Mathematics Subject Classification 33C45 $\cdot 33$ C47 $\cdot 33$ C55 $\cdot 22$ E70

Communicated by Tom H. Koornwinder.

Robert S. Maier

rsm@math.arizona.edu

1 Departments of Mathematics and Physics, University of Arizona, Tucson, AZ 85721, USA 


\section{Introduction}

The first-kind associated Legendre functions $P_{v}^{\mu}(z)$, or the Ferrers versions $\mathrm{P}_{v}^{\mu}(z)$, are classical. $\left(P_{v}^{\mu}(z)\right.$ and $\mathrm{P}_{v}^{\mu}(z)$ are continuations of each other, with respective real domains $z \in(1, \infty)$ and $z \in(-1,1))$. The roles they play when the degree $v$ and order $\mu$ equal integers $n, m$ are familiar. The Legendre, or Ferrers polynomials $\mathrm{P}_{n}(z):=\mathrm{P}_{n}^{0}(z), n=0,1,2, \ldots$, are orthogonal on $[-1,1]$ and are used in series expansions. The spherical harmonics $Y_{n}^{m}(\theta, \phi) \propto \mathrm{P}_{n}^{m}(\cos \theta) \mathrm{e}^{\mathrm{i} m \phi}$ are orthogonal on the symmetric space $S^{2}=S O(3) / S O(2)$, and appear in harmonic analysis based on the Lie group $S O(3)$.

It is less well known that Ferrers functions $\mathrm{P}_{v}^{\mu}(z)$ of a fixed order $\mu$, and degrees that may be nonintegral but are spaced by integers, can also be used in series expansions. The fundamental relation, due to Love and Hunter [21], is one of biorthogonality:

$$
\int_{-1}^{1} \mathrm{P}_{v}^{\mu}(z) \mathrm{P}_{v^{\prime}}^{-\mu}(-z) \mathrm{d} z=0
$$

which holds if (i) $\operatorname{Re} \mu \in(-1,1)$, and (ii) the degrees $v, v^{\prime}$ differ by a nonzero even integer and are not half-odd-integers. For suitable $\nu_{0}, \mu \in \mathbb{C}$, this makes possible bilateral expansions of the form

$$
f(z)=\sum_{n=-\infty}^{\infty} c_{n} \mathrm{P}_{\nu_{0}+2 n}^{\mu}(z), \quad z \in(-1,1),
$$

and in particular, the calculation of the coefficients $c_{n}$ as inner products in $L^{2}[-1,1]$. (This is the usual Legendre expansion if $\left(v_{0}, \mu\right)=(0,0)$, as $\mathrm{P}_{-v-1}=\mathrm{P}_{v}$ for all v.) For conditions on $f$ sufficient for (interior) pointwise convergence, see $[20,21]$.

The restriction to $\operatorname{Re} \mu \in(-1,1)$ comes from the requirement that the expansion functions lie in $L^{2}[-1,1]$. If the order $\mu$ is not a positive integer, $\mathrm{P}_{v}^{\mu}(z)$ will have leading behavior as $z \rightarrow 1^{-}$proportional to $(1-z)^{-\mu / 2}$, but in general its leading behavior as $z \rightarrow(-1)^{+}$comprises two terms: one proportional to $(1+z)^{-\mu / 2}$, and one to $(1+z)^{+\mu / 2}$. The implications for convergence of the integral in (1.1) are obvious. These asymptotics have motivated the suggestion by Pinsky [32] that when $\operatorname{Re} \mu<0$, the series (1.2) should really be viewed as an expansion of [ $(1-z) /$ $(1+z)]^{\mu / 2} f(z)$ in the functions $[(1-z) /(1+z)]^{\mu / 2} \mathrm{P}_{\nu_{0}+2 n}^{\mu}(z)$. This enables a discussion of endpoint convergence, because the latter functions do not diverge as $z \rightarrow 1^{-},(-1)^{+}$.

It is not usually the case that $\mathrm{P}_{\nu}^{\mu}(z)$ and $P_{v}^{\mu}(z)$ are elementary functions, unless of course $v$ and $\mu$ are integers. This may be why such expansions as (1.2) have been used infrequently. In this paper, we derive explicit, trigonometrically parametrized formulas for several families of Legendre functions, expressing $\mathrm{P}_{v}^{\mu}(z), P_{v}^{\mu}(z)$, and their second-kind counterparts $\mathrm{Q}_{v}^{\mu}(z), Q_{v}^{\mu}(z)$, as elementary functions. In each family, $\nu, \mu$ are nonintegral but are spaced by integers: $(\nu, \mu) \in\left(v_{0}, \mu_{0}\right)+\mathbb{Z}^{2}$ for some fractional $\nu_{0}, \mu_{0}$. 
The simplest example is

$$
\begin{aligned}
\mathrm{P}_{-\frac{1}{6}+n}^{\frac{1}{4}+m}(\cos \theta)= & 2^{-2 m-3 n} \Gamma\left(\frac{3}{4}-m\right)^{-1} \\
& \times(\sin \theta)^{-\frac{1}{4}-m} B_{+}^{\frac{1}{4}+3 m+3 n} r_{n}^{m}\left(B_{-} / B_{+}\right), \quad \theta \in(0, \pi), \\
B_{ \pm}= & B_{ \pm}(\theta):=\cos (\theta / 3) \pm \sqrt{\frac{4 \cos ^{2}(\theta / 3)-1}{3}},
\end{aligned}
$$

where $(n, m) \in \mathbb{Z}^{2}$. Here, $r_{n}^{m}=r_{n}^{m}(u)$ is an 'octahedral' rational function that if $n, m \geq 0$ is a polynomial of degree $3 n+2 m$ in $u$; in the base case $n=m=0$, it equals unity. It satisfies differential recurrences on $n$ and $m$, and three-term nondifferential recurrences as well.

The function $r_{0}^{m}(u)$ has a hypergeometric representation in the Gauss function ${ }_{2} F_{1}$ : it equals ${ }_{2} F_{1}\left(-2 m,-\frac{1}{4}-3 m ; \frac{3}{4}-m \mid u\right)$. But $r_{n}^{0}(u)$, which according to (1.3) appears in series of the form (1.2) when $\mu=\frac{1}{4}$, is less classical. It satisfies a secondorder differential equation on the Riemann $u$-sphere with four singular points, not three; so (if $n \geq 0$ ) it is a Heun polynomial, not a hypergeometric one. The functions $\left\{r_{n}^{0}(u)\right\}_{n \in \mathbb{Z}}$ are mutually orthogonal on the $u$-interval $[0,1]$, in a sense that follows from (1.1), but the orthogonality is of an unusual Sturm-Liouville kind.

It is clear from (1.3) that for any $n, m \in \mathbb{Z}$, the function $\mathrm{P}_{-\frac{1}{6}+n}^{\frac{1}{4}+m}(z=\cos \theta)$ depends algebraically on $z$ and can be evaluated using radicals. Each of the function families considered in this paper is similarly algebraic, and because any Legendre function can be written in terms of ${ }_{2} F_{1}$, the results below are really trigonometric parametrizations of families of algebraic ${ }_{2} F_{1}$ 's. To see a link to prior work, recall from Frobenius theory that each Legendre function of degree $v$ and order $\mu$ satisfies a differential equation on the Riemann sphere with three singular points, the characteristic exponent differences at which are $\mu, \mu, 2 v+1$. It is a classical result of Schwarz (see [35], and for more recent expositions, [11, Sect. 2.7.2], [33, Chap. VII] and [25]) that any such equation will have only algebraic solutions only if the (unordered, unsigned) triple of exponent differences falls into one of several classes. The triples from $(\nu, \mu)=\left(-\frac{1}{6}, \frac{1}{4}\right)+$ $(n, m)$, as in (1.3), are $\left(\frac{1}{4}, \frac{1}{4}, \frac{2}{3}\right)+(m, m, 2 n)$, and they lie in Schwarz's octahedral class V.

The families treated below include octahedral ones, with $\left(\nu+\frac{1}{2}, \mu\right) \in\left( \pm \frac{1}{3}, \pm \frac{1}{4}\right)+$ $\mathbb{Z}^{2}$, and tetrahedral ones, with $\left(v+\frac{1}{2}, \mu\right) \in\left( \pm \frac{1}{4}, \pm \frac{1}{3}\right)+\mathbb{Z}^{2}$ or $\left( \pm \frac{1}{3}, \pm \frac{1}{3}\right)+\mathbb{Z}^{2}$, the Schwarz classes for the latter being II and III. The resulting Legendre functions are octahedral or tetrahedral in the sense that their defining differential equation, on the Riemann $z$-sphere, has as its projective monodromy group a finite subgroup of the Möbius group $\operatorname{PSL}(2, \mathbb{R})$, which is octahedral or tetrahedral. This will not be developed at length, but there is a strong geometric reason why $\left\{r_{n}^{m}(u)\right\}_{n, m \in \mathbb{Z}}$ deserve to be called octahedral functions, or (when $n, m \geq 0$ ) polynomials. For general $n, m$, the lifted function $\tilde{r}_{n}^{m}=\tilde{r}_{n}^{m}(s):=r_{n}^{m}\left(u=s^{4}\right)$ turns out to satisfy an equation on the Riemann $s$-sphere with 14 singular points. These include $s=0, \pm 1, \pm \mathrm{i}, \infty$, which 
are the six vertices of an octahedron inscribed in the sphere, and also, the centers of its eight faces.

Up to normalization, the doubly indexed functions $r_{n}^{m}(u)$ are identical to specializations of triply-indexed ones introduced by Ochiai and Yoshida in their groundbreaking work on algebraic ${ }_{2} F_{1}$ 's [29]. For Schwarz classes such as the octahedral and tetrahedral, they considered the effects of displacing the triple of exponent differences, not by $(m, m, 2 n)$ as in the Legendre case, but by general elements of $\mathbb{Z}^{3}$. It is a key result of the present paper that in the Legendre case, when the triple has only two degrees of freedom, it is far easier to derive and solve recurrences on exponent displacements.

Schwarz's classification of algebraic ${ }_{2} F_{1}$ 's also includes a dihedral class (class I) and a related 'cyclic' class (unnumbered but called class $\mathrm{O}$ here). Legendre functions lie in class I when the order $\mu$ is a half-odd-integer, and in class $\mathrm{O}$ when the degree $v$ is an integer. We obtain explicit formulas for the Legendre (and Ferrers) functions in the respective families, of the first and second kinds. The simplest dihedral example is

$$
\begin{aligned}
\mathrm{P}_{-\frac{1}{2}+\alpha}^{\frac{1}{2}+m}(\cos \theta)= & \sqrt{\frac{2}{\pi}} m ! \\
& \times(\sin \theta)^{-1 / 2}\left\{\mathrm{i}^{m} \mathrm{e}^{\mathrm{i} \alpha \theta} P_{m}^{(\alpha,-\alpha)}(\mathrm{i} \cot \theta)\right\}_{\alpha,+}, \quad \theta \in(0, \pi),
\end{aligned}
$$

where $m=0,1,2, \ldots$, and $\alpha \in \mathbb{C}$ is arbitrary. Here, $P_{m}^{(\alpha,-\alpha)}$ is the Jacobi polynomial of degree $m$, and $\{\cdot\}_{\alpha,+}$ signifies the even part under $\alpha \mapsto-\alpha$.

When $m=0$, this becomes a trigonometric version of a well-known algebraic formula $[11,3.6(12)]$, and when $\alpha=\frac{1}{2}$, it expresses $\mathrm{P}_{0}^{\frac{1}{2}+m}$ in terms of the $m$ th Chebyshev polynomial of the third kind. But the general Jacobi representation (1.4) is new. There is a significant literature on 'dihedral polynomials' appearing in dihedrally symmetric ${ }_{2} F_{1}$ 's $[29,38]$, and Vidūnas has shown they can be expressed as terminating Appell series [38]. Focusing on the Legendre case, when two of the three exponent differences are equal, leads to such simpler formulas as (1.4), for both the dihedral and cyclic families.

Constructing bilateral Ferrers series of the form (1.2) is facilitated by the explicit formulas derived below for the Legendre and Ferrers functions in the several families. But the functions $\left\{\mathrm{P}_{v_{0}+n}^{\mu_{0}+m}(z=\cos \theta)\right\},(n, m) \in \mathbb{Z}^{2}$, and the corresponding spherical harmonics $\left\{Y_{v_{0}+n}^{\mu_{0}+m}(\theta, \phi)\right\}$, do not fit into conventional $S O(3)$-based harmonic analysis unless $\left(\nu_{0}, \mu_{0}\right)=(0,0)$, when the latter are the usual surface harmonics on $S^{2}=$ $S O(3) / S O(2)$. In the octahedral and tetrahedral families (and also the dihedral and cyclic, if $\nu_{0}$ resp. $\mu_{0}$ is rational), it is nonetheless the case that each spherical harmonic can be viewed as a finite-valued function on $S^{2}$. This is due both to $\nu_{0}, \mu_{0}$ being rational and to the algebraicity of $\mathrm{P}_{\nu_{0}+n}^{\mu_{0}+m}(z)$ in its argument $z$, as seen in (1.3).

To begin to relate the present results to harmonic analysis, we interpret in Lietheoretic terms the recurrences satisfied by any family $\left\{\mathrm{P}_{\nu_{0}+n}^{\mu_{0}+m}(z)\right\}$ or $\left\{Y_{\nu_{0}+n}^{\mu_{0}+m}(\theta, \phi)\right\}$, $(n, m) \in \mathbb{Z}^{2}$, which are based on first-order differential operators that perform ladder operations. It is well known that for any $\left(v_{0}, \mu_{0}\right)$, there is an infinite-dimensional representation of the Lie algebra $\mathfrak{s o}(3, \mathbb{R})$ on the span of $\left\{Y_{\nu_{0}}^{\mu_{0}+m}(z)\right\}_{m \in \mathbb{Z}}$. (In the 
case when $\left(v_{0}, \mu_{0}\right) \in \mathbb{Z}_{\geq} \times \mathbb{Z}$, this includes as an irreducible constituent the familiar $\left(2 v_{0}+1\right)$-dimensional representation carried by the span of $Y_{\nu_{0}}^{-v_{0}}, \ldots, Y_{\nu_{0}}^{\nu_{0}}$.) There is also a representation of $\mathfrak{s o}(2,1)$ on the span of $\left\{Y_{v_{0}+n}^{\mu_{0}}(z)\right\}_{n \in \mathbb{Z}}$. The real algebras $\mathfrak{s o}(3, \mathbb{R}), \mathfrak{s o}(2,1)$ are real forms of the complex Lie algebra $\mathfrak{s o}(3, \mathbb{C})$.

As we explain, these 'order' and 'degree' algebras generate over $\mathbb{C}$ a 10 dimensional, rank-2 complex Lie algebra isomorphic to $\mathfrak{s o}(5, \mathbb{C})$, which for any $\left(\nu_{0}, \mu_{0}\right)$, acts differentially on the family $\left\{r^{\nu_{0}+n} Y_{v_{0}+n}^{\mu_{0}+m}(\theta, \phi)\right\},(n, m) \in \mathbb{Z}^{2}$, of generalized solid harmonics on $\mathbb{R}^{3}$. The root system of $\mathfrak{s o}(5, \mathbb{C})$, of type $B_{2}$, comprises the eight displacement vectors $\Delta(\nu, \mu)=(0, \pm 1),( \pm 1,0),( \pm 1, \pm 1)$, which yield four ladders on $(\nu, \mu)$, and for each ladder, there are differential operators for raising and lowering, a differential recurrence satisfied by $\mathrm{P}_{v}^{\mu}(z=\cos \theta)$, and a three-term nondifferential recurrence. The ones coming from the roots $( \pm 1, \pm 1)$, such as the 'diagonal' recurrences

$$
\begin{aligned}
& \sqrt{1-z^{2}} \mathrm{P}_{v \pm 1}^{\mu+1}(z)+\left[ \pm(2 v+1)\left(1-z^{2}\right)+2 \mu\right] \mathrm{P}_{v}^{\mu}(z) \\
& +\left[\left(v+\frac{1}{2}\right) \pm\left(\mu-\frac{1}{2}\right)\right]\left[\left(v+\frac{1}{2}\right) \pm\left(\mu-\frac{3}{2}\right)\right] \sqrt{1-z^{2}} \mathrm{P}_{v \mp 1}^{\mu-1}(z)=0
\end{aligned}
$$

may be given here for the first time. (In this identity, P may be replaced by Q.)

Connections between associated Legendre/Ferrers functions, or spherical harmonics, and the complex Lie algebra $\mathfrak{s o}(5, \mathbb{C})$ [or its real forms $\mathfrak{s o}(3,2), \mathfrak{s o}(4,1)$, and $\mathfrak{s o}(5, \mathbb{R})]$ are known to exist. (See [27] and [28, Chaps. 3,4], and [4,9] in the physics literature, and also [19] for hyperspherical extensions.) But most work has focused on functions of integral degree and order. The octahedral, tetrahedral, dihedral, and cyclic families yield explicit infinite-dimensional representations of $\mathfrak{s o}(5, \mathbb{C})$ and its real forms, which are carried by finite-valued solid harmonics on $\mathbb{R}^{3}$. When $\left(v_{0}, \mu_{0}\right)=\left(\frac{1}{2}, \frac{1}{2}\right)$ or $(0,0)$, the representation of $\mathfrak{s o}(3,2)$ turns out to include a known skew-Hermitian one, of the Dirac singleton type (the ' $\mathrm{Di}$ ' or the 'Rac' one, respectively). But in general, these Lie algebra representations are new, non-skew-Hermitian ones, which do not integrate to unitary representations of the corresponding Lie group. It is shown below that any of these representations of $\mathfrak{s o}(5, \mathbb{C})$ [or any of its real forms], carried by a harmonic family $\left\{r^{\nu_{0}+n} Y_{v_{0}+n}^{\mu_{0}+m}(\theta, \phi)\right\}$, $(n, m) \in \mathbb{Z}^{2}$, is of a distinguished kind, in the sense that it assigns special values to the two Casimir invariants of the algebra, these values being independent of $\left(v_{0}, \mu_{0}\right)$; cf. $[4,19]$.

This paper is structured as follows. In Sect. 2, facts on Legendre/Ferrers functions that will be needed are reviewed. In Sect. 3, the key results on the octahedral functions $r_{n}^{m}$ are stated, and explicit formulas for octahedral Legendre/Ferrers functions are derived. These are extended to the tetrahedral families in Sects. 4 and 5. In Sect. 6, the results in Sect. 3 are proved. In Sect. 7, Love-Hunter biorthogonality is related to Sturm-Liouville biorthogonality. In Sect. 8, formulas for Legendre/Ferrers functions in the cyclic and dihedral families are derived, and Love-Hunter expansions in dihedral Ferrers functions are briefly explored. In Sect. 9, recurrences on the degree and order, valid for any $\left(\nu_{0}, \mu_{0}\right)$, are derived, and are given a Lie-theoretic interpretation: $\mathfrak{s o}(5, \mathbb{C})$ and its real forms are introduced, and their representations carried by solid harmonics are examined. 


\section{Preliminaries}

The (associated) Legendre equation is the second-order differential equation

$$
\frac{\mathrm{d}}{\mathrm{d} z}\left[\left(1-z^{2}\right) \frac{\mathrm{d} p}{\mathrm{~d} z}\right]+\left[v(v+1)-\frac{\mu^{2}}{1-z^{2}}\right] p=0
$$

on the complex $z$-plane. For there to be single-valued solutions, the plane is cut along the real axis either from $-\infty$ to 1 (the Legendre choice), or from $-\infty$ to -1 and from 1 to $+\infty$ (the Ferrers choice). The respective solution spaces have $P_{v}^{\mu}(z), Q_{v}^{\mu}(z)$ and $\mathrm{P}_{v}^{\mu}(z), \mathrm{Q}_{v}^{\mu}(z)$ as bases, except in degenerate cases indicated below.

At fixed real $\mu$, Eq. (2.1) can be viewed as a singular Sturm-Liouville equation on the real Ferrers domain $[-1,1]$, the endpoints of which are of Weyl's 'limit circle' type if $\mu \in(-1,1)$. (See [13].) In this case, all solutions $p=p(z)$ lie in $L^{2}[-1,1]$, irrespective of $v$, but the same is not true when $\mu \notin(-1,1)$, which is why such orthogonality relations as $(1.1)$ can only be obtained if $\mu \in(-1,1)$, or more generally if $\operatorname{Re} \mu \in(-1,1)$.

Further light on endpoint behavior is shed by Frobenius theory. Equation (2.1) has regular singular points at $z=-1,1$ and $\infty$, with respective characteristic exponents expressed in terms of the degree $v$ and order $\mu$ as $+\mu / 2,-\mu / 2 ;+\mu / 2,-\mu / 2$; and $-v, v+1$. The exponent differences are $\mu, \mu, 2 v+1$. The functions $P_{v}^{\mu}, \mathrm{P}_{v}^{\mu}$ are Frobenius solutions associated with the exponent $-\mu / 2$ at $z=1$, and the second Legendre function $Q_{v}^{\mu}$ is associated with the exponent $v+1$ at $z=\infty$. (The second Ferrers function $Q_{v}^{\mu}$ is a combination of two Frobenius solutions.) These functions are defined to be analytic (or rather meromorphic) in $v, \mu[30]$, the Legendre functions having the normalizations

$$
\begin{array}{ll}
P_{v}^{\mu}(z) \sim \frac{2^{\mu / 2}}{\Gamma(1-\mu)}(z-1)^{-\mu / 2}, & z \rightarrow 1, \\
\hat{Q}_{v}^{\mu}(z) \sim \frac{\sqrt{\pi}}{2^{v+1}} \frac{\Gamma(v+\mu+1)}{\Gamma(v+3 / 2)} z^{-v-1}, & z \rightarrow \infty,
\end{array}
$$

by convention [11, Chap. III]. The notation $\hat{Q}_{v}^{\mu}:=\mathrm{e}^{-\mu \pi \mathrm{i}} Q_{v}^{\mu}$ will be used henceforth; it removes an awkward $\mathrm{e}^{\mu \pi \mathrm{i}}$ factor.

The formulas (2.2a), (2.2b) apply if the gammas are finite, the asymptotics when they are not are given in [31]. One such degenerate case is when $\mu=1,2, \ldots$. Then, $P_{-\mu}^{\mu}, \ldots, P_{\mu-1}^{\mu} \equiv 0$. A familiar example is when the degree is a nonnegative integer $n$. Then, $P_{n}^{m}, \mathrm{P}_{n}^{m} \equiv 0$ if the order is an integer $m>n$, though not if $m<-n$. Another degenerate case is when $v+\mu$ is a negative integer. If so, $\hat{Q}_{v}^{\mu}$ is undefined, as (2.2b) suggests, except when $v=-\frac{3}{2},-\frac{5}{2}, \ldots$ Then, $\hat{Q}_{v}^{v+1}, \ldots, \hat{Q}_{v}^{-(v+1)}$ are defined.

The Ferrers functions are related to the Legendre ones on their common domains, which are the upper and lower half-planes $\pm \operatorname{Im} z>0$, by

$$
\begin{aligned}
\mathrm{P}_{v}^{\mu} & =\mathrm{e}^{ \pm \mu \pi \mathrm{i} / 2} P_{v}^{\mu}, \\
\mathrm{Q}_{v}^{\mu} & =\mathrm{e}^{\mp \mu \pi \mathrm{i} / 2} \hat{Q}_{v}^{\mu} \pm \mathrm{i}(\pi / 2) \mathrm{e}^{ \pm \mu \pi \mathrm{i} / 2} P_{v}^{\mu} .
\end{aligned}
$$


Thus $P_{v}^{\mu}, \mathrm{P}_{v}^{\mu}$ are related by analytic continuation, up to phase. Going from $P_{v}^{\mu}$ to $\mathrm{P}_{v}^{\mu}$ typically involves replacing a factor $(z-1)^{-\mu / 2}$ by $(1-z)^{-\mu / 2}$; for instance, $P_{1}^{-1}(z)$, $\mathrm{P}_{1}^{-1}(z)$ are $\frac{1}{2} \sqrt{z^{2}-1}, \frac{1}{2} \sqrt{1-z^{2}}$. Also, owing to (2.3b), $\mathrm{Q}_{\nu}^{\mu}$ is undefined if and only if $\hat{Q}_{v}^{\mu}$ is.

Equation (2.1) is invariant under $v \mapsto-v-1, \mu \mapsto-\mu$, and $z \mapsto-z$, so that in nondegenerate cases, the Legendre and Ferrers functions with $v$ replaced by $-v-1$, $\mu$ by $-\mu$, and/or $z$ by $-z$, can be expressed as combinations of any two (at most) of $P_{v}^{\mu}, \hat{Q}_{v}^{\mu}, \mathrm{P}_{v}^{\mu}, \mathrm{Q}_{v}^{\mu}$. Some 'connection' formulas of this type, which will be needed below, are $P_{-v-1}^{\mu}=P_{v}^{\mu}, \mathrm{P}_{-v-1}^{\mu}=\mathrm{P}_{v}^{\mu}$,

$$
\hat{Q}_{v}^{-\mu} / \Gamma(v-\mu+1)=\hat{Q}_{v}^{\mu} / \Gamma(v+\mu+1),
$$

the $P \rightarrow \hat{Q}$ reduction

$$
P_{v}^{\mu}=\sec (v \pi) \Gamma(v-\mu+1)^{-1} \Gamma(-\mu-v)^{-1}\left(\hat{Q}_{-v-1}^{-\mu}-\hat{Q}_{v}^{-\mu}\right),
$$

the $\hat{Q} \rightarrow P$ reduction

$$
(2 / \pi) \hat{Q}_{v}^{\mu}=\csc (\mu \pi) P_{v}^{\mu}-\csc (\mu \pi) \frac{\Gamma(v+\mu+1)}{\Gamma(v-\mu+1)} P_{v}^{-\mu}
$$

and the $\mathrm{Q} \rightarrow \mathrm{P}$ reduction

$$
(2 / \pi) \mathrm{Q}_{v}^{\mu}=\cot (\mu \pi) \mathrm{P}_{v}^{\mu}-\csc (\mu \pi) \frac{\Gamma(v+\mu+1)}{\Gamma(v-\mu+1)} \mathrm{P}_{v}^{-\mu}
$$

(See [11].) It follows from (2.7) that if $\mu=\frac{1}{2}, \frac{3}{2}, \ldots$, then $\mathrm{Q}_{-\mu}^{\mu}, \ldots, \mathrm{Q}_{\mu-1}^{\mu} \equiv 0$.

The functions $P_{v}^{\mu}, \hat{Q}_{v}^{\mu}$ are known to have the hypergeometric representations

$$
\begin{aligned}
P_{v}^{\mu}(z)= & \frac{1}{\Gamma(1-\mu)}\left(\frac{z+1}{z-1}\right)^{\mu / 2}{ }_{2} F_{1}\left(-v, v+1 ; 1-\mu ; \frac{1-z}{2}\right) \\
= & \frac{2^{\mu}}{\Gamma(1-\mu)} \frac{z^{v+\mu}}{\left(z^{2}-1\right)^{\mu / 2}}{ }_{2} F_{1}\left(-\frac{v}{2}-\frac{\mu}{2},-\frac{v}{2}-\frac{\mu}{2}+\frac{1}{2} ; 1-\mu ; 1-\frac{1}{z^{2}}\right), \\
\hat{Q}_{v}^{\mu}(z)= & \frac{\sqrt{\pi}}{2^{v+1}} \frac{\Gamma(v+\mu+1)}{\Gamma(v+3 / 2)} \frac{(z+1)^{\mu / 2}}{(z-1)^{\mu / 2+v+1}} \\
& \times{ }_{2} F_{1}\left(v+1, v+\mu+1 ; 2 v+2 ; \frac{2}{1-z}\right) \\
= & \frac{\sqrt{\pi}}{2^{v+1}} \frac{\Gamma(v+\mu+1)}{\Gamma(v+3 / 2)} \frac{\left(z^{2}-1\right)^{\mu / 2}}{z^{v+\mu+1}} \\
& \times{ }_{2} F_{1}\left(\frac{v}{2}+\frac{\mu}{2}+\frac{1}{2}, \frac{v}{2}+\frac{\mu}{2}+1 ; v+\frac{3}{2} ; \frac{1}{z^{2}}\right) .
\end{aligned}
$$


(For $\mathrm{P}_{v}^{\mu}$, replace $z-1$ in the prefactor on the right of (2.8a) by $1-z$; the alternative expressions $(2.8 \mathrm{~b}),(2.8 \mathrm{~d})$ come from $(2.8 \mathrm{a}),(2.8 \mathrm{c})$ by quadratic hypergeometric transformations.) Here, ${ }_{2} F_{1}(a, b ; c ; x)$ is the Gauss function with parameters $a, b ; c$, defined (on the disk $|x|<1$, at least) by the Maclaurin series $\sum_{k=0}^{\infty}\left[(a)_{k}(b)_{k} /(c)_{k}(1)_{k}\right] x^{k}$. In this and below, the notation $(d)_{k}$ is used for the rising factorial, i.e.,

$$
(d)_{k}:=, \begin{cases}(d) \ldots(d+k-1), & k \geq 0, \\ {\left[\left(d-k^{\prime}\right) \ldots(d-1)\right]^{-1},} & k=-k^{\prime} \leq 0 .\end{cases}
$$

(The unusual second half of this definition, which extends the meaning of $(d)_{k}$ to negative $k$ so that $(d)_{k}=\left[(d+k)_{-k}\right]^{-1}$ for all $k \in \mathbb{Z}$, will be needed below.) If in any ${ }_{2} F_{1}(a, b ; c ; x)$ in $(2.8)$, the denominator parameter $c$ is a nonpositive integer, and there is an apparent division by zero, the taking of a limit is to be understood.

The Gauss equation satisfied by ${ }_{2} F_{1}(a, b ; c ; x)$ has the three singular points $x=0,1, \infty$, with respective exponent differences $1-c, c-a-b, b-a$. Taking into account either of (2.8a), (2.8c), one sees that this triple is consistent with the exponent differences $\mu, \mu, 2 v+1$ at the singular points $z=1,-1, \infty$ of the Legendre equation (2.1). Schwarz's results on algebraicity were originally phrased in terms of the Gauss equation, its solutions such as ${ }_{2} F_{1}$, and the (unordered, unsigned) triple $1-c, c-a-b, b-a$, but they extend to the Legendre equation, its solutions, and the triple $\mu, \mu, 2 v+1$.

\section{Octahedral Formulas (Schwarz Class V)}

This section and Sects. 4 and 5 derive parametric formulas for Legendre and Ferrers functions that are either octahedral or tetrahedral (two types). The formulas involve the octahedral polynomials, or functions, $\left\{r_{n}^{m}(u)\right\}_{n, m \in \mathbb{Z}}$. Section 3.1 defines these rational functions and states several results, the proofs of which are deferred to Sect. 6.

\subsection{Indexed Functions and Polynomials}

Definition 3.1 For $n, m \in \mathbb{Z}$, the rational functions $r_{n}^{m}=r_{n}^{m}(u)$ and their 'conjugates' $\bar{r}_{n}^{m}=\bar{r}_{n}^{m}(u)$ are defined implicitly by

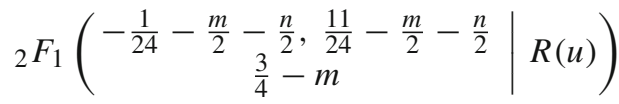

$$
\begin{aligned}
& =\left[(1+u)\left(1-34 u+u^{2}\right)\right]^{-1 / 12-m-n} r_{n}^{m}(u), \\
& { }_{2} F_{1}\left(\begin{array}{c|c}
\frac{5}{24}+\frac{m}{2}-\frac{n}{2}, \frac{17}{24}+\frac{m}{2}-\frac{n}{2} & R(u)) \\
\frac{5}{4}+m &
\end{array}\right. \\
& =\left[(1+u)\left(1-34 u+u^{2}\right)\right]^{5 / 12+m-n}(1-u)^{-1-4 m} \bar{r}_{n}^{m}(u),
\end{aligned}
$$


which hold on a neighborhood of $u=0$. Here,

$$
\begin{aligned}
R(u) & :=\frac{-108 u(1-u)^{4}}{\left[(1+u)\left(1-34 u+u^{2}\right)\right]^{2}}=1-\frac{\left(1+14 u+u^{2}\right)^{3}}{\left[(1+u)\left(1-34 u+u^{2}\right)\right]^{2}}, \\
& :=-108 \frac{p_{\mathrm{v}}(u)}{p_{\mathrm{e}}(u)^{2}}=1-\frac{p_{\mathrm{f}}(u)^{3}}{p_{\mathrm{e}}(u)^{2}}
\end{aligned}
$$

where $p_{\mathrm{v}}, p_{\mathrm{e}}, p_{\mathrm{f}}$ are the polynomials $u(1-u)^{4},(1+u)\left(1-34 u+u^{2}\right), 1+14 u+u^{2}$, which satisfy $p_{\mathrm{e}}^{2}-p_{\mathrm{f}}^{3}+108 p_{\mathrm{v}}=0$. Equivalently,

$$
R=\frac{T(3+T)^{2}}{(1+3 T)^{2}}=1-\frac{(1-T)^{3}}{(1+3 T)^{2}}
$$

where $T=T(u):=-12 u /(1+u)^{2}$.

[For later use, note that (3.1) is familiar from trigonometry as a 'triple-angle' formula: $R=\tanh ^{2} \xi$ if $T=\tanh ^{2}(\xi / 3) ; R=\operatorname{coth}^{2} \xi$ if $T=\operatorname{coth}^{2}(\xi / 3)$; and $R=-\tan ^{2} \theta$ if $T=-\tan ^{2}(\theta / 3)$.]

It is clear from the definition that $r_{n}^{m}, \bar{r}_{n}^{m}$ are analytic at $u=0$, at which they equal unity, though it is not obvious that they are rational in $u$. But it is easily checked that the Gauss equations satisfied by the two ${ }_{2} F_{1}(x)$ functions have respective exponent differences (at the singular points $x=0,1, \infty)$ equal to $\left(\frac{1}{4}, \frac{1}{3}, \frac{1}{2}\right)+(m, n, 0)$ and $\left(-\frac{1}{4}, \frac{1}{3}, \frac{1}{2}\right)+(-m, n, 0)$. These triples lie in Schwarz's octahedral class IV, so each ${ }_{2} F_{1}(x)$ must be an algebraic function of $x$. The definition implicitly asserts that if these algebraic ${ }_{2} F_{1}$ 's are parametrized by the degree- 6 rational function $x=R(u)$, the resulting dependences on $u$ will be captured by certain rational $r_{n}^{m}=r_{n}^{m}(u)$, $\bar{r}_{n}^{m}=\bar{r}_{n}^{m}(u)$. In the terminology of [29], these are octahedral functions of $u$.

Theorem 3.1 (i) For $n, m \geq 0, r_{n}^{m}(u)$ is a polynomial of degree $3 n+2 m$ in $u$, to be called the octahedral polynomial indexed by $(n, m) \in \mathbb{Z}_{\geq}^{2}$. Its coefficient of $u^{0}$ is unity and its coefficient of $u^{3 n+2 m}$ is

$$
d_{n}^{m}:=(-)^{m+n} 3^{3 m} \frac{\left(\frac{5}{12}\right)_{m-n}\left(\frac{13}{12}\right)_{m+n}}{\left(\frac{1}{4}\right)_{m}\left(\frac{5}{4}\right)_{m}} .
$$

(ii) For unrestricted $(n, m) \in \mathbb{Z}^{2}, r_{n}^{m}(u)$ is a rational function that equals unity at $u=0$ and is asymptotic to $d_{n}^{m} u^{3 n+2 m}$ as $u \rightarrow \infty$.

(iii) The conjugate function $\bar{r}_{n}^{m}$ is related to $r_{n}^{m}$ by

$$
\bar{r}_{n}^{m}(u)=\left(d_{n}^{m}\right)^{-1} u^{3 n+2 m} r_{n}^{m}(1 / u),
$$

so that if $n, m \geq 0, \bar{r}_{n}^{m}$ is a reversed version of the polynomial $r_{n}^{m}$, scaled to equal unity at $u=0$. 
The functional form of the octahedral functions that are not polynomials, which are indexed by $(n, m) \in \mathbb{Z}^{2} \backslash \mathbb{Z}_{\geq}^{2}$, is not complicated.

Theorem 3.2 For any $n, m \geq 0$,

$$
\begin{aligned}
r_{n}^{m}(u) & =\Pi_{3 n+2 m}(u), \\
r_{n}^{-m-1}(u) & =(1-u)^{-3-4 m} \Pi_{1+3 n+2 m}(u), \\
r_{-n-1}^{m}(u) & =\left(1+14 u+u^{2}\right)^{-2-3 n} \Pi_{1+3 n+2 m}(u), \\
r_{-n-1}^{-m-1}(u) & =(1-u)^{-3-4 m}\left(1+14 u+u^{2}\right)^{-2-3 n} \Pi_{2+3 n+2 m}(u),
\end{aligned}
$$

where on each line, $\Pi_{k}(u)$ signifies a polynomial of degree $k$ in $u$, with its coefficient of $u^{0}$ equalling unity and its coefficient of $u^{k}$ coming from the preceding theorem.

On their indices $n, m$, the $r_{n}^{m}$ satisfy both differential recurrences and three-term nondifferential recurrences. The former are given in Sect. 6 (see Theorem 6.1), and the latter are as follows.

Theorem 3.3 The octahedral functions $r_{n}^{m}=r_{n}^{m}(u)$, indexed by $(n, m) \in \mathbb{Z}^{2}$, satisfy second-order (i.e., three-term) recurrences on $m$ and n; namely,

$$
\begin{aligned}
& (4 m-3)(4 m+1) r_{n}^{m+1}-(4 m-3)(4 m+1) p_{\mathrm{e}}(u) r_{n}^{m} \\
& \quad-3(12 m-12 n-7)(12 m+12 n+1) p_{\mathrm{v}}(u) r_{n}^{m-1}=0 \\
& (12 n-12 m+7) r_{n+1}^{m}-8(3 n+1) p_{\mathrm{e}}(u) r_{n}^{m} \\
& \quad+(12 n+12 m+1) p_{\mathrm{f}}^{3}(u) r_{n-1}^{m}=0
\end{aligned}
$$

where $p_{\mathrm{v}}, p_{\mathrm{e}}, p_{\mathrm{f}}$ are the polynomials in $u$, satisfying $p_{\mathrm{e}}^{2}-p_{\mathrm{f}}^{3}+108 p_{\mathrm{v}}=0$, that were introduced in Definition 3.1. Moreover, they satisfy

$$
\begin{aligned}
& 3(4 m-3)(4 m+1) r_{n+1}^{m+1}-(4 m-3)\left[(12 m+12 n+7) p_{\mathrm{f}}^{3}(u)-4(3 n+1) p_{\mathrm{e}}^{2}(u)\right] r_{n}^{m} \\
& \quad+9(12 m+12 n+1)(12 m+12 n-11) p_{\mathrm{v}}(u) p_{\mathrm{f}}^{3}(u) r_{n-1}^{m-1}=0, \\
& 3(4 m-3)(4 m+1) p_{\mathrm{f}}^{3}(u) r_{n-1}^{m+1}-(4 m-3)\left[(12 m-12 n-1) p_{\mathrm{f}}^{3}(u)+4(3 n+1) p_{\mathrm{e}}^{2}(u)\right] r_{n}^{m} \\
& \quad+9(12 m-12 n-7)(12 m-12 n-19) p_{\mathrm{v}}(u) r_{n+1}^{m-1}=0,
\end{aligned}
$$

which are second-order 'diagonal' recurrences.

From the first two recurrences in this theorem, one can compute $r_{n}^{m}$ for any $n, m \in \mathbb{Z}$, if one begins with $r_{0}^{0}, r_{0}^{1}, r_{1}^{0}$, which are low-degree polynomials in $u$ computable 'by hand.' In fact,

$$
\begin{aligned}
& r_{0}^{0}(u)=1, \quad r_{0}^{1}(u)=1-26 u-39 u^{2}, \quad r_{1}^{0}(u)=1-39 u-\frac{195}{7} u^{2}+\frac{13}{7} u^{3} \\
& r_{1}^{1}(u)=1+175 u-150 u^{2}+3550 u^{3}+325 u^{4}+195 u^{5}
\end{aligned}
$$

By specializing to $u=1$ (at which $p_{\mathrm{v}}, p_{\mathrm{e}}, p_{\mathrm{f}}$ equal $0,-64,36$ ), one can prove by induction that $r_{n}^{m}(1)=(-64)^{m+n}$ if $m \geq 0$. Examples of octahedral functions that are 
not polynomials because they have at least one negative index, illustrating Theorem 3.2, are

$$
\begin{aligned}
& r_{0}^{-1}(u)=(1-u)^{-3}\left(1+\frac{1}{7} u\right) \\
& r_{-1}^{0}(u)=\left(1+14 u+u^{2}\right)^{-2}(1-5 u) \\
& r_{-1}^{-1}(u)=(1-u)^{-3}\left(1+14 u+u^{2}\right)^{-2}\left(1+2 u-\frac{1}{11} u^{2}\right) .
\end{aligned}
$$

These also follow from the recurrences of Theorem 3.3.

The recurrences are nonclassical, not least because they are bilateral: they extend to $n, m<0$. It is shown in Sect. 6 that for $n, m \geq 0$, the degree- $(3 n+2 m)$ polynomials $r_{n}^{m}$ in $u$ are (generalized) Heun polynomials, rather than hypergeometric ones; they are not orthogonal polynomials in the conventional sense. A useful third-order (i.e., four-term) recurrence on $k$ for the coefficients $\left\{a_{k}\right\}_{k=0}^{3 n+2 m}$ of $r_{n}^{m}$ is given in Theorem 6.4.

An important degenerate case is worth noting: the case $n=0$. For any $m \geq 0$, there are hypergeometric representations in ${ }_{2} F_{1}$ for the degree- $2 m$ octahedral polynomials $r_{0}^{m}$ and $\bar{r}_{0}^{m} ;$ namely,

$$
r_{0}^{m}(u)={ }_{2} F_{1}\left(\begin{array}{c|c}
-2 m,-\frac{1}{4}-3 m & u \\
\frac{3}{4}-m & u
\end{array}\right), \quad \bar{r}_{0}^{m}(u)={ }_{2} F_{1}\left(\begin{array}{c}
-2 m, \frac{1}{4}-m \\
\frac{5}{4}+m
\end{array} \mid u\right) .
$$

These follow by a sextic hypergeometric transformation of the ${ }_{2} F_{1}$ 's in Definition 1 , as well as by the methods of Sect. 6 . The first can also be deduced from the $n=0$ case of the recurrence on $m$ in Theorem 3.3. These representations extend to $m \in \mathbb{Z}$.

\subsection{Explicit Formulas}

The following two theorems (Theorems 3.4 and 3.5) give trigonometrically parametrized formulas for the Legendre/Ferrers functions $P_{v}^{\mu}, \mathrm{P}_{v}^{\mu}$ when $(\nu, \mu)$ equals $\left(-\frac{1}{6}, \frac{1}{4}\right)+(n, m)$ and $\left(-\frac{1}{6},-\frac{1}{4}\right)+(n,-m)$, with $(n, m) \in \mathbb{Z}^{2}$. The triple of exponent differences $(\mu, \mu, 2 v+1)$ is respectively equal to $\left(\frac{1}{4}, \frac{1}{4}, \frac{2}{3}\right)+(m, m, 2 n)$ and $\left(-\frac{1}{4},-\frac{1}{4}, \frac{2}{3}\right)+(-m,-m, 2 n)$, both lying in Schwarz's octahedral class V. An interesting application of these formulas to the evaluation of certain Mehler-Dirichlet integrals appears in Theorem 3.6.

Let hyperbolic-trigonometric functions $A_{ \pm}$, positive on $(0, \infty)$, be defined by

$$
\begin{aligned}
A_{ \pm}=A_{ \pm}(\xi) & := \pm \cosh (\xi / 3)+\sqrt{\frac{\sinh \xi}{3 \sinh (\xi / 3)}} \\
& = \pm \cosh (\xi / 3)+\sqrt{\frac{4 \cosh ^{2}(\xi / 3)-1}{3}}
\end{aligned}
$$

so that $A_{+} A_{-}(\xi)=\frac{1}{3} \sinh ^{2}(\xi / 3)$. This choice is motivated by Definition 3.1: if $R=R(u)$ and $T=T(u)=-12 u /(1+u)^{2}$ are alternatively parametrized as $\tanh ^{2} \xi$ 
and $\tanh ^{2}(\xi / 3)$, respectively, it is not difficult to verify that the three polynomials in $u$ that appear in Definition 3.1 will have the $\xi$-parametrizations

$$
\begin{array}{ll}
p_{\mathrm{v}}=u(1-u)^{4} & =-\frac{16}{27} A_{+}^{-6} \sinh ^{2} \xi, \\
p_{\mathrm{e}}=(1+u)\left(1-34 u+u^{2}\right) & =8 A_{+}^{-3} \cosh \xi, \\
p_{\mathrm{f}}=1+14 u+u^{2} & =4 A_{+}^{-2} .
\end{array}
$$

Moreover, and more fundamentally, $u=-A_{-} / A_{+}$.

Also in this section, let $\hat{r}_{n}^{m}$ signify $r_{n}^{m} / d_{n}^{m}$, so that when $n, m \geq 0, \hat{r}_{n}^{m}$ is a scaled version of the octahedral polynomial $r_{n}^{m}$, with its leading rather than its trailing coefficient equal to unity. Equivalently, $\hat{r}_{n}^{m}(u)=u^{3 n+2 m} \bar{r}_{n}^{m}(1 / u)$.

\section{Theorem 3.4 The formulas}

$$
\begin{aligned}
P_{-\frac{1}{6}+n}^{\frac{1}{4}+m}(\cosh \xi)= & {\left[2^{-2 m-3 n} \Gamma\left(\frac{3}{4}-m\right)^{-1}\right] } \\
& \times(\sinh \xi)^{-1 / 4-m} A_{+}^{1 / 4+3 m+3 n} r_{n}^{m}\left(-A_{-} / A_{+}\right), \\
P_{-\frac{1}{6}+n}^{-\frac{1}{4}-m}(\cosh \xi)= & {\left[(-)^{n} 2^{-2 m-3 n} 3^{3 / 4+3 m} \Gamma\left(\frac{5}{4}+m\right)^{-1}\right] } \\
& \times(\sinh \xi)^{-1 / 4-m} A_{-}^{1 / 4+3 m+3 n} \hat{r}_{n}^{m}\left(-A_{+} / A_{-}\right)
\end{aligned}
$$

hold for $(n, m) \in \mathbb{Z}^{2}$ and $\xi \in(0, \infty)$.

[Note that as $\xi$ increases from 0 to $\infty$, the argument $u=-A_{-} / A_{+}$of the first $r_{n}^{m}$, which satisfies $T(u)=\tanh ^{2}(\xi / 3)$ and $R(u)=\tanh ^{2} \xi$, decreases from 0 to $-(2-\sqrt{3})^{2}$ $\approx-0.07$, which is a root of $p_{\mathrm{f}}(u)=1+14 u+u^{2}$.]

Proof These formulas follow from the hypergeometric representation (2.8b) of $P_{v}^{\mu}=$ $P_{v}^{\mu}(z)$, together with the implicit definitions of $r_{n}^{m}, \bar{r}_{n}^{m}$ (see Definition 3.1).

If $z=\cosh \xi$, the argument $1-1 / z^{2}$ of the right-hand ${ }_{2} F_{1}$ in $(2.8 \mathrm{~b})$ will equal $\tanh ^{2} \xi$. This is why it is natural to parametrize Definition 3.1 by letting $R=R(u)$ equal $\tanh ^{2} \xi$, with the just-described consequences. In deriving the formulas, one needs the representation (3.6b), and for the second formula, the definition (3.2) of $d_{n}^{m}$.

In the following, the circular-trigonometric functions $B_{ \pm}$, positive on $(0, \pi)$, are defined by

$$
\begin{aligned}
B_{ \pm}=B_{ \pm}(\theta) & :=\cos (\theta / 3) \pm \sqrt{\frac{\sin \theta}{3 \sin (\theta / 3)}} \\
& =\cos (\theta / 3) \pm \sqrt{\frac{4 \cos ^{2}(\theta / 3)-1}{3}}
\end{aligned}
$$

so that $B_{+} B_{-}(\theta)=\frac{1}{3} \sin ^{2}(\theta / 3)$. 


\section{Theorem 3.5 The formulas}

$$
\begin{aligned}
\mathrm{P}_{-\frac{1}{6}+n}^{\frac{1}{4}+m}(\cos \theta)= & {\left[2^{-2 m-3 n} \Gamma\left(\frac{3}{4}-m\right)^{-1}\right] } \\
& \times(\sin \theta)^{-1 / 4-m} B_{+}^{1 / 4+3 m+3 n} r_{n}^{m}\left(B_{-} / B_{+}\right), \\
\mathrm{P}_{-\frac{1}{6}+n}^{-\frac{1}{4}-m}(\cos \theta)= & {\left[2^{-2 m-3 n} 3^{3 / 4+3 m} \Gamma\left(\frac{5}{4}+m\right)^{-1}\right] } \\
& \times(\sin \theta)^{-1 / 4-m} B_{-}^{1 / 4+3 m+3 n} \hat{r}_{n}^{m}\left(B_{+} / B_{-}\right)
\end{aligned}
$$

hold for $(n, m) \in \mathbb{Z}^{2}$ and $\theta \in(0, \pi)$.

[Note that as $\theta$ increases from 0 to $\pi$, the argument $u=B_{-} / B_{+}$of the first $r_{n}^{m}$, which satisfies $T(u)=-\tan ^{2}(\theta / 3)$ and $R(u)=-\tan ^{2} \theta$, increases from 0 to 1.]

Proof The proof is accomplished by analytic continuation of Theorem 3.4, or in effect, by letting $\xi=\mathrm{i} \theta$.

Because $P_{v}^{\mu}=P_{-v-1}^{\mu}$ and $\mathrm{P}_{v}^{\mu}=\mathrm{P}_{-v-1}^{\mu}$, Theorems 3.4 and 3.5 also supply formulas for $P_{-\frac{5}{6}-n}^{\frac{1}{4}+m}, P_{-\frac{5}{6}-n}^{-\frac{1}{4}-m}$ and $\mathrm{P}_{-\frac{5}{6}-n}^{\frac{1}{4}+m}, \mathrm{P}_{-\frac{5}{6}-n}^{-\frac{1}{4}-m}$. By exploiting the $\hat{Q} \rightarrow P$ and $\mathrm{Q} \rightarrow \mathrm{P}$ reductions (2.6) and (2.7), one easily obtains additional formulas, for $\hat{Q}_{-\frac{1}{6}+n}^{\frac{1}{4}+m}, \hat{Q}_{-\frac{1}{6}+n}^{-\frac{1}{4}-m}$, $\hat{Q}_{-\frac{5}{6}-n}^{\frac{1}{4}+m}, \hat{Q}_{-\frac{5}{6}-n}^{-\frac{1}{4}-m}$ and $\mathrm{Q}_{-\frac{1}{6}+n}^{\frac{1}{4}+m}, \mathrm{Q}_{-\frac{1}{6}+n}^{-\frac{1}{4}-m}, \mathrm{Q}_{-\frac{5}{6}-n}^{\frac{1}{4}+m}, \mathrm{Q}_{-\frac{5}{6}-n}^{-\frac{1}{4}-m}$.

Theorems 3.4 and 3.5 permit certain Mehler-Dirichlet integrals to be evaluated in closed form. For example, consider [11, 3.7(8) and 3.7(27)]

$$
\begin{aligned}
P_{\nu}^{\mu}(\cosh \xi) & =\sqrt{\frac{2}{\pi}} \frac{(\sinh \xi)^{\mu}}{\Gamma\left(\frac{1}{2}-\mu\right)} \int_{0}^{\xi} \frac{\cosh \left[\left(v+\frac{1}{2}\right) t\right]}{(\cosh \xi-\cosh t)^{\mu+\frac{1}{2}}} \mathrm{~d} t \\
\mathrm{P}_{\nu}^{\mu}(\cos \theta) & =\sqrt{\frac{2}{\pi}} \frac{(\sin \theta)^{\mu}}{\Gamma\left(\frac{1}{2}-\mu\right)} \int_{0}^{\theta} \frac{\cos \left[\left(\nu+\frac{1}{2}\right) \phi\right]}{(\cos \phi-\cos \theta)^{\mu+\frac{1}{2}}} \mathrm{~d} \phi,
\end{aligned}
$$

which hold when $\operatorname{Re} \mu<\frac{1}{2}$ for $\xi \in(0, \infty)$ and $\theta \in(0, \pi)$. These integral representations of the Legendre and Ferrers functions of the first kind are classical $[16,22]$.

\section{Theorem 3.6 The formulas}

$$
\begin{gathered}
\int_{0}^{\xi} \frac{\cosh \left[\left(\frac{1}{3}+n\right) t\right]}{(\cosh \xi-\cosh t)^{\frac{1}{4}-m}} \mathrm{~d} t=(-1)^{n} K_{n}^{m} A_{-}^{1 / 4+3 m+3 n} \hat{r}_{n}^{m}\left(-A_{+} / A_{-}\right), \\
\int_{0}^{\theta} \frac{\cos \left[\left(\frac{1}{3}+n\right) \phi\right]}{(\cos \phi-\cos \theta)^{\frac{1}{4}-m}} \mathrm{~d} \phi=K_{n}^{m} B_{-}^{1 / 4+3 m+3 n} \hat{r}_{n}^{m}\left(B_{+} / B_{-}\right),
\end{gathered}
$$


with

$$
K_{n}^{m}=\sqrt{\frac{\pi}{2}} 2^{-2 m-3 n} 3^{3 / 4+3 m} \frac{\Gamma\left(\frac{3}{4}+m\right)}{\Gamma\left(\frac{5}{4}+m\right)}
$$

hold when $m$ is a nonnegative integer and $n$ an integer, for $\xi \in(0, \infty)$ and $\theta \in(0, \pi)$.

Proof Substitute the second formulas of Theorems 3.4 and 3.5 into the $(\nu, \mu)=$ $\left(-\frac{1}{6}+n,-\frac{1}{4}-m\right)$ specializations of (3.7) and (3.8).

\section{Tetrahedral Formulas (Schwarz Class II)}

The following theorem gives trigonometrically parametrized formulas for the second Legendre function $\hat{Q}_{v}^{\mu}$ when $(v, \mu)=\left(-\frac{3}{4},-\frac{1}{3}\right)+(-m,-n)$ and $\left(-\frac{1}{4},-\frac{1}{3}\right)+$ $(m,-n)$, with $(n, m) \in \mathbb{Z}^{2}$. The triple of exponent differences, $(\mu, \mu, 2 v+1)$, is respectively equal to $\left(-\frac{1}{3},-\frac{1}{3},-\frac{1}{2}\right)+(-n,-n,-2 m)$ and $\left(-\frac{1}{3},-\frac{1}{3}, \frac{1}{2}\right)+$ $(-n,-n, 2 m)$, both lying in Schwarz's tetrahedral class II. The hyperbolictrigonometric functions $A_{ \pm}=A_{ \pm}(\xi)$ on $(0, \infty)$ are defined as in (3.5).

Theorem 4.1 The formulas

$$
\begin{aligned}
& (2 / \pi) \hat{Q}_{-\frac{3}{4}-m}^{-\frac{1}{3}-n}(\operatorname{coth} \xi)=\left[2^{11 / 4-2 m-3 n} 3^{-3 / 8}\left(\frac{1}{4}\right)_{m}\left(\frac{13}{12}\right)_{m+n}^{-1} \Gamma\left(\frac{4}{3}\right)^{-1}\right] \\
& \times(\sinh \xi)^{1 / 4-m}\left[-(-)^{n} \sqrt{\sqrt{3}+1} A_{+}^{1 / 4+3 m+3 n} r_{n}^{m}\left(-A_{-} / A_{+}\right)\right], \\
& (2 / \pi) \hat{Q}_{-\frac{1}{4}+m}^{-\frac{1}{3}-n}(\operatorname{coth} \xi)=\left[2^{11 / 4-2 m-3 n} 3^{-3 / 8}\left(\frac{1}{4}\right)_{m}\left(\frac{13}{12}\right)_{m+n}^{-1} \Gamma\left(\frac{4}{3}\right)^{-1}\right] \\
& \times(\sinh \xi)^{1 / 4-m}\left[+(-)^{m} \sqrt{\sqrt{3}-1} A_{-}^{1 / 4+3 m+3 n} r_{n}^{m}\left(-A_{+} / A_{-}\right)\right]
\end{aligned}
$$

hold for $(n, m) \in \mathbb{Z}^{2}$ and $\xi \in(0, \infty)$.

Proof Combine Whipple's $\hat{Q} \rightarrow P$ transformation [11, 3.3(13)],

$$
\hat{Q}_{v}^{\mu}(\operatorname{coth} \xi)=\sqrt{\pi / 2} \Gamma(\nu+\mu+1)(\sinh \xi)^{1 / 2} P_{-\mu-\frac{1}{2}}^{-v-\frac{1}{2}}(\cosh \xi)
$$

with the results in Theorem 3.4. The symmetrical forms of the right-hand prefactors are obtained with the aid of the gamma-function identities

$$
\begin{aligned}
\sqrt{\sqrt{3}+1} & =\pi^{1 / 2} 2^{1 / 4} 3^{-3 / 8} \Gamma(1 / 12) \Gamma(1 / 4)^{-1} \Gamma(1 / 3)^{-1} \\
& =\pi^{-3 / 2} 2^{-3 / 4} 3^{3 / 8} \Gamma(11 / 12) \Gamma(1 / 4) \Gamma(1 / 3)
\end{aligned}
$$




$$
\begin{aligned}
\sqrt{\sqrt{3}-1} & =\pi^{-1 / 2} 2^{-1 / 4} 3^{1 / 8} \Gamma(5 / 12) \Gamma(1 / 4)^{-1} \Gamma(1 / 3) \\
& =\pi^{-1 / 2} 2^{-1 / 4} 3^{-1 / 8} \Gamma(7 / 12) \Gamma(1 / 4) \Gamma(1 / 3)^{-1}
\end{aligned}
$$

of Vidūnas [37].

Because $\hat{Q}_{\nu}^{\mu}, \hat{Q}_{\nu}^{-\mu}$ are proportional to each other (see (2.4)), Theorem 4.1 also supplies formulas for $\hat{Q}_{-\frac{3}{4}-m}^{\frac{1}{3}+n}, \hat{Q}_{-\frac{1}{4}+m}^{\frac{1}{3}+n}$. Moreover, it leads to the following two theorems.

Theorem 4.2 The formulas

$$
\begin{aligned}
& P_{-\frac{3}{4}-m}^{-\frac{1}{3}-n}(\operatorname{coth} \xi)=\left[(-)^{n} 2^{5 / 4-2 m-3 n} 3^{-3 / 8}\left(\frac{1}{4}\right)_{m}\left(\frac{13}{12}\right)_{m+n}^{-1} \Gamma\left(\frac{4}{3}\right)^{-1}\right] \\
& \times(\sinh \xi)^{1 / 4-m}\left[(-)^{n} \sqrt{\sqrt{3}-1} A_{+}^{1 / 4+3 m+3 n} r_{n}^{m}\left(-A_{-} / A_{+}\right)\right. \\
& \left.-(-)^{m} \sqrt{\sqrt{3}+1} A_{-}^{1 / 4+3 m+3 n} r_{n}^{m}\left(-A_{+} / A_{-}\right)\right], \\
& P_{-\frac{3}{4}-m}^{\frac{1}{3}+n}(\operatorname{coth} \xi)=\left[(-)^{n} 2^{-1 / 4-2 m-3 n} 3^{-3 / 8}\left(\frac{1}{4}\right)_{m}\left(\frac{5}{12}\right)_{m-n}^{-1} \Gamma\left(\frac{2}{3}\right)^{-1}\right] \\
& \times(\sinh \xi)^{1 / 4-m}\left[(-)^{n} \sqrt{\sqrt{3}+1} A_{+}^{1 / 4+3 m+3 n} r_{n}^{m}\left(-A_{-} / A_{+}\right)\right. \\
& \left.+(-)^{m} \sqrt{\sqrt{3}-1} A_{-}^{1 / 4+3 m+3 n} r_{n}^{m}\left(-A_{+} / A_{-}\right)\right]
\end{aligned}
$$

hold for $(n, m) \in \mathbb{Z}^{2}$ and $\xi \in(0, \infty)$.

Proof Combine the $P \rightarrow \hat{Q}$ reduction (2.5) with the results in Theorem 4.1.

Because $P_{v}^{\mu}=P_{-v-1}^{\mu}$, Theorem 4.2 also supplies formulas for $P_{-\frac{1}{4}+m}^{-\frac{1}{3}-n}, P_{-\frac{1}{4}+m}^{\frac{1}{3}+n}$.

In Theorem 4.3, the hyperbolic-trigonometric functions $C_{ \pm}$, positive on $(-\infty, \infty)$, are defined by

$$
\begin{aligned}
C_{ \pm}=C_{ \pm}(\xi) & := \pm \sinh (\xi / 3)+\sqrt{\frac{\cosh \xi}{3 \cosh (\xi / 3)}} \\
& = \pm \sinh (\xi / 3)+\sqrt{\frac{4 \sinh ^{2}(\xi / 3)+1}{3}}
\end{aligned}
$$

so that $C_{+} C_{-}(\xi)=\frac{1}{3} \cosh ^{2}(\xi / 3)$.

Theorem 4.3 The formulas

$$
\begin{aligned}
& \mathrm{P}_{-\frac{3}{4}-m}^{-\frac{1}{3}-n}(\tanh \xi)=\left[2^{5 / 4-2 m-3 n} 3^{-3 / 8}\left(\frac{1}{4}\right)_{m}\left(\frac{13}{12}\right)_{m+n}^{-1} \Gamma\left(\frac{4}{3}\right)^{-1}\right] \\
& \quad \times(\cosh \xi)^{1 / 4-m}\left[-(-)^{n} \sqrt{\sqrt{3}-1} C_{+}^{1 / 4+3 m+3 n} r_{n}^{m}\left(-C_{-} / C_{+}\right)\right.
\end{aligned}
$$




$$
\begin{aligned}
& \left.+(-)^{m} \sqrt{\sqrt{3}+1} C_{-}^{1 / 4+3 m+3 n} r_{n}^{m}\left(-C_{+} / C_{-}\right)\right] \\
& \mathrm{P}_{-\frac{3}{4}-m}^{\frac{1}{3}+n}(\tanh \xi)=\left[(-)^{n} 2^{-1 / 4-2 m-3 n} 3^{-3 / 8}\left(\frac{1}{4}\right)_{m}\left(\frac{5}{12}\right)_{m-n}^{-1} \Gamma\left(\frac{2}{3}\right)^{-1}\right] \\
& \quad \times(\cosh \xi)^{1 / 4-m}\left[(-)^{n} \sqrt{\sqrt{3}+1} C_{+}^{1 / 4+3 m+3 n} r_{n}^{m}\left(-C_{-} / C_{+}\right)\right. \\
& \left.+(-)^{m} \sqrt{\sqrt{3}-1} C_{-}^{1 / 4+3 m+3 n} r_{n}^{m}\left(-C_{+} / C_{-}\right)\right]
\end{aligned}
$$

hold for $(n, m) \in \mathbb{Z}^{2}$ and $\xi \in(-\infty, \infty)$.

[Note that as $\xi$ decreases from $\infty$ to $-\infty$, the argument $u=-C_{-} / C_{+}$of the first $r_{n}^{m}$, which satisfies $T(u)=\operatorname{coth}^{2}(\xi / 3)$ and $R(u)=\operatorname{coth}^{2} \xi$, decreases from 0 to $-(2+\sqrt{3})^{2} \approx-14.0$, which is a root of $p_{\mathrm{f}}(u)=1+14 u+u^{2}$.]

Proof The proof is accomplished by analytic continuation of the results in Theorem 4.2 , or in effect, by replacing $\xi$ by $\xi+\mathrm{i} \pi / 2$.

Because $\mathrm{P}_{v}^{\mu}=\mathrm{P}_{-v-1}^{\mu}$, Theorem 4.3 also supplies formulas for $\mathrm{P}_{-\frac{1}{4}+m}^{-\frac{1}{3}-n}, \mathrm{P}_{-\frac{1}{4}+m}^{\frac{1}{3}+n}$. By exploiting the $\mathrm{Q} \rightarrow \mathrm{P}$ reduction (2.7), one easily obtains additional formulas, for $\mathrm{Q}_{-\frac{3}{4}-m}^{-\frac{1}{3}-n}, \mathrm{Q}_{-\frac{3}{4}-m}^{\frac{1}{3}+n}, \mathrm{Q}_{-\frac{1}{4}+m}^{-\frac{1}{3}-n}, \mathrm{Q}_{-\frac{1}{4}+m}^{\frac{1}{3}+n}$

\section{Tetrahedral Formulas (Schwarz Class III)}

The following theorems give parametrized formulas for the Legendre/Ferrers functions $P_{v}^{\mu}, \hat{Q}_{\nu}^{\mu}, \mathrm{P}_{v}^{\mu}$ when $(v, \mu)=\left(-\frac{1}{6},-\frac{1}{3}\right)+(n,-n)$ and $\left(-\frac{5}{6}, \frac{1}{3}\right)+(-n, n)$, with $n \in \mathbb{Z}$. The triple of exponent differences, $(\mu, \mu, 2 v+1)$, is respectively equal to $\left(-\frac{1}{3},-\frac{1}{3}, \frac{2}{3}\right)+(-n,-n, 2 n)$ and $\left(\frac{1}{3}, \frac{1}{3},-\frac{2}{3}\right)+(n, n,-2 n)$, both lying in Schwarz's tetrahedral class III.

Theorem 5.1 The formulas

$$
\begin{aligned}
& \mathrm{P}_{-\frac{1}{6}+n}^{-\frac{1}{3}-n}\left(\sqrt{1-\mathrm{e}^{-2 \xi}}\right)=2^{-\frac{1}{3}-n}\left(1-\mathrm{e}^{-2 \xi}\right)^{-1 / 4} P_{-\frac{3}{4}}^{-\frac{1}{3}-n}(\operatorname{coth} \xi), \\
& \mathrm{P}_{-\frac{5}{6}-n}^{\frac{1}{3}+n}\left(\sqrt{1-\mathrm{e}^{-2 \xi}}\right)=2^{\frac{1}{3}+n}\left(1-\mathrm{e}^{-2 \xi}\right)^{-1 / 4} P_{-\frac{3}{4}}^{\frac{1}{3}+n}(\operatorname{coth} \xi),
\end{aligned}
$$

where expressions for the right-hand Legendre functions are provided by Theorem 4.2, hold for $n \in \mathbb{Z}$ and $\xi \in(0, \infty)$.

Theorem 5.2 The formulas

$$
\begin{aligned}
& P_{-\frac{1}{6}+n}^{-\frac{1}{3}-n}\left(\sqrt{1+\mathrm{e}^{-2 \xi}}\right)=2^{-\frac{1}{3}-n}\left(1+\mathrm{e}^{-2 \xi}\right)^{-1 / 4} \mathrm{P}_{-\frac{3}{4}}^{-\frac{1}{3}-n}(\tanh \xi), \\
& P_{-\frac{5}{6}-n}^{\frac{1}{3}+n}\left(\sqrt{1+\mathrm{e}^{-2 \xi}}\right)=2^{\frac{1}{3}+n}\left(1+\mathrm{e}^{-2 \xi}\right)^{-1 / 4} \mathrm{P}_{-\frac{3}{4}}^{\frac{1}{3}+n}(\tanh \xi),
\end{aligned}
$$


where expressions for the right-hand Ferrers functions are provided by Theorem 4.3, hold for $n \in \mathbb{Z}$ and $\xi \in(-\infty, \infty)$.

Theorem 5.3 The formulas

$$
\begin{aligned}
& (2 / \pi) \hat{Q}_{-\frac{1}{6}+n}^{-\frac{1}{3}-n}\left(\sqrt{1+\mathrm{e}^{2 \xi}}\right)=2^{-\frac{1}{3}-n}\left(1+\mathrm{e}^{2 \xi}\right)^{-1 / 4} \sqrt{2} \mathrm{P}_{-\frac{3}{4}}^{-\frac{1}{3}-n}(-\tanh \xi), \\
& (2 / \pi) \hat{Q}_{-\frac{5}{6}-n}^{\frac{1}{3}+n}\left(\sqrt{1+\mathrm{e}^{2 \xi}}\right)=2^{\frac{1}{3}+n}\left(1+\mathrm{e}^{2 \xi}\right)^{-1 / 4} \sqrt{2} \mathrm{P}_{-\frac{3}{4}}^{\frac{1}{3}+n}(-\tanh \xi),
\end{aligned}
$$

where expressions for the right-hand Ferrers functions are provided by Theorem 4.3, hold for $n \in \mathbb{Z}$ and $\xi \in(-\infty, \infty)$.

Because $P_{v}^{\mu}=P_{-v-1}^{\mu}$ and $\mathrm{P}_{v}^{\mu}=\mathrm{P}_{-v-1}^{\mu}$, Theorems 5.1 and 5.2 also supply formulas for $P_{-\frac{5}{6}-n}^{-\frac{1}{3}-n}, P_{-\frac{1}{6}+n}^{\frac{1}{3}+n}, \mathrm{P}_{-\frac{5}{6}-n}^{-\frac{1}{3}-n}, \mathrm{P}_{-\frac{1}{6}+n}^{\frac{1}{3}+n}$. And because $\hat{Q}_{\nu}^{\mu}, \hat{Q}_{\nu}^{-\mu}$ are proportional to each other (see (2.4)), Theorem 5.3 also supplies formulas for $\hat{Q}_{-\frac{1}{6}+n}^{\frac{1}{3}+n}, \hat{Q}_{-\frac{5}{6}-n}^{-\frac{1}{3}-n}$. By exploiting the $\mathrm{Q} \rightarrow \mathrm{P}$ reduction (2.7), one easily obtains additional formulas, for $\mathrm{Q}_{-\frac{1}{6}+n}^{-\frac{1}{3}-n}, \mathrm{Q}_{-\frac{5}{6}-n}^{\frac{1}{3}+n}, \mathrm{Q}_{-\frac{5}{6}-n}^{-\frac{1}{3}-n}, \mathrm{Q}_{-\frac{1}{6}+n}^{\frac{1}{3}+n}$

The formulas in Theorems 5.1, 5.2, and 5.3 are straightforward reparametrizations of the $\alpha=\frac{1}{3}+n$ and $\alpha=-\frac{1}{3}-n$ cases of the identities

$$
\begin{aligned}
P_{-\frac{1}{4}}^{-\alpha}(\cosh \xi) & =2^{\alpha} \sqrt{\operatorname{sech}(\xi / 2)} \mathrm{P}_{\alpha-\frac{1}{2}}^{-\alpha}(\operatorname{sech}(\xi / 2)), \\
\mathrm{P}_{-\frac{1}{4}}^{-\alpha}(\cos \theta) & =2^{\alpha} \sqrt{\sec (\theta / 2)} P_{\alpha-\frac{1}{2}}^{-\alpha}(\sec (\theta / 2)), \\
\sqrt{2} \mathrm{P}_{-\frac{1}{4}}^{-\alpha}(-\cos \theta) & =2^{\alpha} \sqrt{\sec (\theta / 2)}(2 / \pi) \hat{Q}_{\alpha-\frac{1}{2}}^{-\alpha}(\sec (\theta / 2)),
\end{aligned}
$$

which hold when $\xi \in(0, \infty)$ and $\theta \in(0, \pi)$. These appear as identities $I_{4}(i), I_{4}(i i)$, and $I_{4}(\overline{i i})$ in [23] and are really quadratic hypergeometric transformations in disguise.

\section{Proofs of Results in Sect. 3.1}

The octahedral and tetrahedral formulas in Sects.3.2, 4, and 5 followed from the theorems in Sect. 3.1 on the octahedral functions $r_{n}^{m}=r_{n}^{m}(u)$, which were stated without proof. The present section provides proofs, in some cases sketched, and obtains a few additional results. These are Theorem 6.1 (on the differential equation and differential recurrences satisfied by $r_{n}^{m}$ ), and Theorems 6.2, 6.3, and 6.4 (on the interpretation of $r_{n}^{m}$ when $n, m \geq 0$ as a hypergeometric, Heun, or generalized Heun polynomial). This section also reveals the origin of the degree-6 rational function $x=R(u)$ in Definition 3.1 .

Consider a Riemann sphere $\mathbb{P}_{s}^{1}$, parametrized by $s$ and identified by stereographic projection with the complex $s$-plane. (As usual, $s=0$ is at the bottom and $s=\infty$ is at the top; points with $|s|=1$ are taken to lie on the equator.) Let a regular octahedron (a Platonic solid) be inscribed in the sphere, with its six vertices $v_{1}, \ldots, v_{6}$ 
at $s=0, \pm 1, \pm \mathrm{i}, \infty$, i.e., at the five roots of $q_{\mathrm{v}}(s):=s\left(1-s^{4}\right)$ and at $s=\infty$. By some trigonometry [33, chap. VII], one can show that the twelve edge-midpoints $e_{1}, \ldots, e_{12}$ of the octahedron, radially projected onto the sphere, are located at $s=$ $( \pm 1 \pm \mathrm{i}) / \sqrt{2}$ and $s=\sqrt{ \pm 1}( \pm 1 \pm \sqrt{2})$, which are the roots of $q_{\mathrm{e}}:=\left(1+s^{4}\right)$ $\left(1-34 s^{4}+s^{8}\right)=1-33 s^{4}-33 s^{8}+s^{12}$. Similarly, its eight face-centers $f_{1}, \ldots f_{8}$, when radially projected, are located at $s=( \pm 1 \pm \mathrm{i})(1 \pm \sqrt{3}) / 2$, which are the roots of $q_{\mathrm{f}}(s):=1+14 s^{4}+s^{8}$. The polynomials $q_{\mathrm{v}}, q_{\mathrm{e}}, q_{\mathrm{f}}$ are (relative) invariants of the symmetry group of the octahedron, which is an order-24 subgroup of the group of rotations of the Riemann $s$-sphere.

The well-known octahedral equation states that $q_{\mathrm{e}}^{2}-q_{\mathrm{f}}^{3}+108 q_{\mathrm{v}}^{4}=0$. The validity of this identity (a syzygy, in the language of invariant theory) suggests considering the degree-24 rational function $\tilde{R}=\tilde{R}(s)$ equal to $1-q_{\mathrm{f}}^{3} / q_{\mathrm{e}}^{2}$; i.e.,

$$
\tilde{R}(s):=\frac{-108\left[s\left(1-s^{4}\right)\right]^{4}}{\left[\left(1+s^{4}\right)\left(1-34 s^{4}+s^{8}\right)\right]^{2}}=1-\frac{\left(1+14 s^{4}+s^{8}\right)^{3}}{\left[\left(1+s^{4}\right)\left(1-34 s^{4}+s^{8}\right)\right]^{2}} .
$$

On the $s$-sphere, $\tilde{R}(s)$ equals $0,1, \infty$ at (respectively) the vertices, the face-centers, and the edge-midpoints. It is an absolute invariant of the symmetry group of the octahedron. (Its derivative $\mathrm{d} \tilde{R}(s) / \mathrm{d} s$ can be written as $-432 q_{\mathrm{v}}^{3} q_{\mathrm{f}}^{2} / q_{\mathrm{e}}^{3}$ but is only a relative invariant.) The covering $\mathbb{P}_{s}^{1} \rightarrow \mathbb{P}_{x}^{1}$ given by $x=\tilde{R}(s)$ is ramified above $x=0,1, \infty$, and its ramification structure can be written as $(6) 4=(8) 3=(12) 2$ : each of the six points above $s=0$ (i.e., the vertices) appears with multiplicity 4 , etc.

Following and extending Schwarz $[33,35]$, consider the effect of lifting the Gauss hypergeometric equation satisfied by ${ }_{2} F_{1}\left(-\frac{1}{24}-\frac{m}{2}-\frac{n}{2}, \frac{11}{24}-\frac{m}{2}-\frac{n}{2} ; \frac{3}{4}-m ; x\right)$, the ${ }_{2} F_{1}(x)$ appearing in Definition 3.1, from the $x$-sphere to the $s$-sphere, along $x=$ $\tilde{R}(s)$. It should be recalled that the Gauss equation satisfied by $f(x)={ }_{2} F_{1}(a, b ; c ; x)$ is the Fuchsian differential equation $\left(D_{x}:=\mathrm{d} / \mathrm{d} x\right)$

$$
D_{x}^{2} f+\left[\frac{c}{x}+\frac{a+b-c+1}{x-1}\right] D_{x} f+\left[\frac{a b}{x(x-1)}\right] f=0,
$$

which has characteristic exponents $\{0,1-c\},\{0, c-a-b\},\{a, b\}$ at its singular points $x=0,1, \infty$, with (unsigned) exponent differences $1-c ; c-a-b ; b-a$. (The function ${ }_{2} F_{1}(a, b ; c ; x)$ is the Frobenius solution associated with the zero exponent at $x=0$.) The effects of the ramified lifting by $x=\tilde{R}(s)$ are conveniently expressed in the classical notation of Riemann P-symbols, which display the exponents at each singular point $[33,39]$. For the ${ }_{2} F_{1}$ of Definition 3.1 , one can write

$$
\begin{aligned}
{ }_{2} F_{1}(x=\tilde{R}(s)) & =P\left\{\begin{array}{ccc|c|}
0 & 1 & \infty & x=\tilde{R}(s) \\
\hline 0 & 0 & -\frac{1}{24}-\frac{m}{2}-\frac{n}{2} & \\
\frac{1}{4}+m & \frac{1}{3}+n & \frac{11}{24}-\frac{m}{2}-\frac{n}{2}
\end{array}\right\} \\
& =P\left\{\begin{array}{ccc|}
\frac{v_{1}, \ldots, v_{6}}{f_{1}, \ldots, f_{8}} & e_{1}, \ldots, e_{12} & s \\
0 & 0 & -\frac{1}{12}-m-n \\
1+4 m & 1+3 n & \frac{11}{12}-m-n
\end{array}\right\},
\end{aligned}
$$


because any pair of characteristic exponents at a point $x=x_{0}$ beneath a ramification point $s=s_{0}$ of order $k$ is multiplied by $k$ when lifted. This function of $s$ satisfies a differential equation on the $s$-sphere with the indicated singular points and exponents.

If $\mathcal{L} g=0$ is any Fuchsian differential equation on the $s$-sphere, the modified equation $\mathcal{L}^{\prime} g^{\prime}=0$ obtained by the change of dependent variable $g^{\prime}=\left(1-s / s_{0}\right)^{\alpha} g$ has its exponents at $s=s_{0}$ shifted upward by $\alpha$, and those at $s=\infty$ shifted downward by the same. As an application of this, one deduces from (6.2b) that

$$
\begin{aligned}
\tilde{f}(s) & =\left[q_{\mathrm{e}}(s)\right]^{\frac{1}{12}+m+n}{ }_{2} F_{1}(x=\tilde{R}(s)) \\
& =P\left\{\begin{array}{cccc|c}
v_{1}, \ldots, v_{5} & f_{1}, \ldots, f_{8} & e_{1}, \ldots, e_{12} & \infty & s \\
0 & 0 & 0 & -1-12 m-12 n & \\
1+4 m & 1+3 n & 1 & -8 m-12 n
\end{array}\right\} \\
& =P\left\{\begin{array}{ccc|c}
v_{1}, \ldots, v_{5} & f_{1}, \ldots, f_{8} & \infty & s \\
0 & 0 & -1-12 m-12 n \\
1+4 m & 1+3 n & -8 m-12 n
\end{array}\right\},
\end{aligned}
$$

because $e_{1}, \ldots, e_{12}$ are the roots of $q_{\mathrm{e}}$, and $v_{6}=\infty$. The left-hand function $\tilde{f}=\tilde{f}(s)$, which by examination is $\tilde{r}_{n}^{m}(s):=r_{n}^{m}\left(u=s^{4}\right)$, will be the solution of a 'lifted and shifted' differential equation on the $s$-sphere, with the indicated exponents. After the shifting, the edge-midpoints $e_{1}, \ldots, e_{12}$ cease being singular points, because the new exponents at each are 0,1 , which are those of an ordinary point.

It is straightforward if tedious to compute the differential equation satisfied by $\tilde{f}=\tilde{r}_{n}^{m}(s):=r_{n}^{m}\left(u=s^{4}\right)$ explicitly, by applying to the appropriate Gauss equation of the form (6.1) the changes of variable that perform (i) the lifting along $s \mapsto x=\tilde{R}(s)$, and (ii) the multiplication by $\left[q_{\mathrm{e}}(s)\right]^{\frac{1}{12}+m+n}$. One finds that $\tilde{f}$ satisfies $\tilde{\mathcal{L}}_{n}^{m} \tilde{f}=0$, where

$$
\begin{aligned}
\tilde{\mathcal{L}}_{n}^{m}= & D_{s}^{2}-\left[4 m \frac{5 s^{4}-1}{s\left(s^{4}-1\right)}+3 n \frac{8 s^{7}+56 s^{3}}{s^{8}+14 s^{4}+1}\right] D_{s} \\
& +4(12 m+12 n+1) \frac{s^{3}\left[(2 m+3 n)\left(s^{8}+14 s^{4}+1\right)-12 n\left(3 s^{4}+1\right)\right]}{s\left(s^{4}-1\right)\left(s^{8}+14 s^{4}+1\right)} .
\end{aligned}
$$

That the singular points of this operator are the roots $v_{1}, \ldots, v_{5}$ of $q_{\mathrm{v}}$ (plus $v_{6}=\infty$ ), and the roots $f_{1}, \ldots, f_{8}$ of $q_{\mathrm{f}}$, is clear, as is the fact that their exponents are as shown in the P-symbol (6.3c). The degenerate case $n=m=0$ is especially interesting. As one expects from the P-symbol, the operator $\tilde{\mathcal{L}}_{0}^{0}$ is simply the Laplacian $D_{s}^{2}$, the kernel of which is spanned by $1, s$. For $\tilde{f}=\tilde{f}(s)=r_{n}^{m}\left(s^{4}\right)$, it is easy to rule out any admixture of the latter solution by examining Definition 3.1, and because $r_{0}^{0}(u)$ equals unity at $u=0$, the base octahedral function $r_{0}^{0}$ must be identically equal to unity.

Because $r_{0}^{0} \equiv 1$, it follows from Definition 3.1 that the hypergeometric function appearing in the definition of $r_{n}^{m}$ when $n=m=0$, which is ${ }_{2} F_{1}\left(-\frac{1}{24}, \frac{11}{24} ; \frac{3}{4} \mid x\right)$, must be algebraic in its argument $x$. This is essentially the 1873 result of Schwarz [35], the proof of which was later restated in a P-symbol form by Poole [33]. However, it 
is not trivial to extend this result on $r_{0}^{0}$ to a constructive proof that $r_{n}^{m}=r_{n}^{m}(u)$ is a rational function of $u$ for each $(n, m) \in \mathbb{Z}^{2}$. This is because the differential equation $\tilde{\mathcal{L}}_{n}^{m} \tilde{f}=0$, as one sees from (6.4), is far more complicated than $D_{s}^{2} \tilde{f}=0$ (Laplace's equation) when $(n, m) \neq(0,0)$. A constructive proof is best based on contiguity relations between adjacent $(n, m)$, i.e., recurrences in the spirit of Gauss, derived as follows.

First, simplify the lifting along the covering $s \mapsto x$, i.e., along the degree-24 map $x=\tilde{R}(s)$. Each octahedral function $\tilde{r}_{n}^{m}(s)$ turns out to 'factor through' $u=s^{4}$, so it suffices to lift the Gauss hypergeometric equation from the $x$-sphere $\mathbb{P}_{x}^{1}$ to the $u$-sphere $\mathbb{P}_{u}^{1}$, along the degree-6 map $x=R(u)$ of Definition 3.1; i.e.,

$$
R(u):=-108 \frac{p_{\mathrm{v}}(u)}{p_{\mathrm{e}}(u)^{2}}=1-\frac{p_{\mathrm{f}}(u)^{3}}{p_{\mathrm{e}}(u)^{2}}
$$

where

$$
\begin{aligned}
& p_{\mathrm{v}}(u)=q_{\mathrm{v}}(s)^{4}=u(1-u)^{4} \\
& p_{\mathrm{e}}(u)=q_{\mathrm{e}}(s)=(1+u)\left(1-34 u+u^{2}\right) \\
& p_{\mathrm{f}}(u)=q_{\mathrm{f}}(s)=1+14 u+u^{2} .
\end{aligned}
$$

Replacing the lifted variable $s$ by $u=s^{4}$ quotients out an order-4 cyclic group of rotations of the $s$-sphere (and hence of the octahedron), about the axis passing through its north and south poles.

The syzygy becomes $p_{\mathrm{e}}^{2}-p_{\mathrm{f}}^{3}+108 p_{\mathrm{v}}=0$. The roots $u=0,1$ of $p_{\mathrm{v}}$, and $u=\infty$, correspond to the south-pole vertex, the four equatorial ones, and the north-pole one. The three roots $u=(3+2 \sqrt{2})^{2},-1,(3-2 \sqrt{2})^{2}$ of $p_{\mathrm{e}}$ correspond to the four edgemidpoints in the northern hemisphere, the four on the equator, and the four to the south. The two roots $u=-(2 \pm \sqrt{3})^{2}$ of $p_{\mathrm{f}}^{2}$ correspond to the four face-centers in the north and the four in the south. The covering $\mathbb{P}_{u}^{1} \rightarrow \mathbb{P}_{x}^{1}$ is still ramified above $x=0,1, \infty$, but its ramification structure is $1+4+1=(2) 3=(3) 2$.

Taking the multiplicities in this ramification structure into account, one finds that if the ${ }_{2} F_{1}$ of Definition 3.1 is lifted along $x=R(u)$ rather than $x=\tilde{R}(s)$, the P-symbol identity (6.3) is replaced by

$$
\begin{aligned}
r_{n}^{m}(u) & =\left[p_{\mathrm{e}}(u)\right]^{\frac{1}{12}+m+n}{ }_{2} F_{1}(x=R(u)) \\
& =P\left\{\begin{array}{cccc|c|}
0 & 1 & -(2 \pm \sqrt{3})^{2} & \infty & u \\
\hline 0 & 0 & 0 & -\frac{1}{4}-3 m-3 n \\
\frac{1}{4}+m & 1+4 m & 1+3 n & -2 m-3 n
\end{array}\right\}
\end{aligned}
$$

This P-symbol has five singular points (at most; fewer if $n=0$ or $m=0$ ). By the preceding explanation, the points $u=0,1, \infty$ represent $1,4,1$ vertices of the octahedron, and each of $u=-(2 \pm \sqrt{3})^{2}$ represents a cycle of four face-centers. 
Theorem 6.1 The octahedral function $r_{n}^{m}=r_{n}^{m}(u)$ satisfies the Fuchsian differential equation $\mathcal{L}_{n}^{m} r_{n}^{m}=0$, where

$$
\begin{aligned}
\mathcal{L}_{n}^{m}=D_{u}^{2} & +\left[\frac{3 / 4-m}{u}+\frac{-4 m}{u-1}+\frac{-3 n(2 u+14)}{u^{2}+14 u+1}\right] D_{u} \\
& +(1+12 m+12 n) \frac{(2 m+3 n)\left(u^{2}+14 u+1\right)-12 n(3 u+1)}{4 u(u-1)\left(u^{2}+14 u+1\right)},
\end{aligned}
$$

the P-symbol of which appears in Eq. (6.5b). The function $r_{n}^{m}$ is the Frobenius solution associated with the zero characteristic exponent of the singular point $u=0$. It satisfies eight differential recurrences of the form

$$
K r_{n+\Delta n}^{m+\Delta m}=p_{\mathrm{v}}^{\left(-\sigma_{\mathrm{v}}+\varepsilon_{\mathrm{v}}\right) / 4} p_{\mathrm{e}}^{-\sigma_{\mathrm{e}}+\varepsilon_{\mathrm{e}}} p_{\mathrm{f}}^{-\sigma_{\mathrm{f}}+\varepsilon_{\mathrm{f}}}\left(4 u^{3 / 4}\right) \frac{\mathrm{d}}{\mathrm{d} u}\left[p_{\mathrm{v}}^{\sigma_{\mathrm{v}} / 4} p_{\mathrm{e}}^{\sigma_{\mathrm{e}}} p_{\mathrm{f}}^{\sigma_{\mathrm{f}}} \cdot r_{n}^{m}\right],
$$

in which $\Delta(n, m)=(0, \pm 1),( \pm 1,0)$ and $( \pm 1, \pm 1)$. For each recurrence, the exponents $\sigma_{\mathrm{v}}, \sigma_{\mathrm{e}}, \sigma_{\mathrm{f}}$, the exponents $\varepsilon_{\mathrm{V}}, \varepsilon_{\mathrm{e}}, \varepsilon_{\mathrm{f}}$, and the prefactor $K$ are listed in Table 1.

Proof The differential equation comes by applying to the appropriate Gauss equation of the form (6.1) the changes of variable that perform (i) the lifting along $u \mapsto x=$ $R(u)$, and (ii) the multiplication by $\left[p_{\mathrm{e}}(u)\right]^{\frac{1}{12}+m+n}$. Or, one can merely substitute $u=s^{4}$ into the equation $\tilde{\mathcal{L}}_{n}^{m} \tilde{r}_{n}^{m}=0$, with $\tilde{\mathcal{L}}_{n}^{m}$ as in (6.4).

The differential recurrences of Jacobi, which shift the parameters $a, b, c$ of the function ${ }_{2} F_{1}(a, b ; c ; x)$ by integers, are well known. (See [11,2.8(20)-2.8(27)].) And if $\Delta(n, m)=( \pm 1, \pm 1)$, the ${ }_{2} F_{1}(R(u))$ in the definition of $r_{n}^{m}(u)$ has its parameters shifted by integers. (See Definition 3.1.) By some calculus, one can change the independent variable in the relevant differential recurrences of Jacobi from $x=R(u)$ to $u$, thereby obtaining the final four recurrences in Table 1 (the diagonal ones). The change uses the fact that $u^{3 / 4} \mathrm{~d} R / \mathrm{d} u$ equals $-108 p_{\mathrm{v}}^{3 / 4} p_{\mathrm{f}}^{2} / p_{\mathrm{e}}^{3}$, and the details are straightforward.

The first four recurrences in the table, with $\Delta(n, m)=(0, \pm 1),( \pm 1,0)$, come a bit less easily, because they shift the ${ }_{2} F_{1}$ parameters in Definition 3.1 by half-integers rather than integers. But by examination, Definition 3.1 is equivalent to

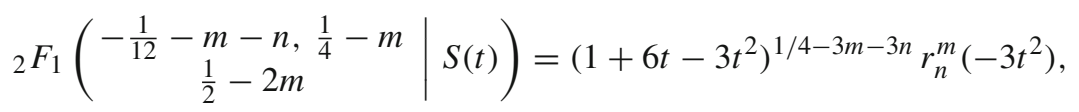

where

$$
S(t):=\frac{36 t\left(1+3 t^{2}\right)^{2}}{\left(1+6 t-3 t^{2}\right)^{3}}=1-\left(\frac{1-6 t-3 t^{2}}{1+6 t-3 t^{2}}\right)^{3}
$$

This follows by a quadratic hypergeometric transformation, $u$ being related to $t$ by $u=-3 t^{2}$, and $R$ to $S$ by $R=S^{2} /(S-2)^{2}$. When $\Delta(n, m)=(0, \pm 1)$ or $( \pm 1,0)$, the parameters of the ${ }_{2} F_{1}$ in (6.6) are shifted by integers, and the same technique can be applied. 
Table 1 Parameters for the differential recurrences of Theorem 6.1

\begin{tabular}{llll}
\hline$\Delta(n, m)$ & $\sigma_{\mathrm{v}}, \sigma_{\mathrm{e}}, \sigma_{\mathrm{f}}$ & $\varepsilon_{\mathrm{V}}, \varepsilon_{\mathrm{e}}, \varepsilon_{\mathrm{f}}$ & $K$ \\
\hline$(0,+1)$ & $-1-4 m, 0, \frac{5}{8}+\frac{3}{2} m-\frac{3}{2} n$ & $1,0,1$ & $-(1+4 m)$ \\
$(0,-1)$ & $0,0,-\frac{1}{8}-\frac{3}{2} m-\frac{3}{2} n$ & $-3,0,1$ & $\frac{3(1+12 m+12 n)(7-12 m+12 n)}{4 m-3}$ \\
$(+1,0)$ & $\frac{7}{6}-2 m+2 n, 0,-1-3 n$ & $1,0,1$ & $\frac{1}{6}(7-12 m+12 n)$ \\
$(-1,0)$ & $-\frac{1}{6}-2 m-2 n, 0,0$ & $1,0,-2$ & $-\frac{1}{6}(1+12 m+12 n)$ \\
$(+1,+1)$ & $-1-4 m, \frac{13}{12}+m+n,-1-3 n$ & $1,1,1$ & $-(1+4 m)$ \\
$(-1,-1)$ & $0,-\frac{1}{12}-m-n, 0$ & $-3,1,-2$ & $\frac{3(1+12 m+12 n)(-11+12 m+12 n)}{4 m-3}$ \\
$(-1,+1)$ & $-1-4 m, \frac{5}{12}+m-n, 0$ & $1,1,-2$ & $-(1+4 m)$ \\
$(+1,-1)$ & $0, \frac{7}{12}-m+n,-1-3 n$ & $-3,1,1$ & $\frac{3(-7+12 m-12 n)(-19+12 m-12 n)}{4 m-3}$ \\
\hline
\end{tabular}

The four three-term nondifferential recurrences in Theorem 3.3 follow by a familiar elimination procedure from the differential recurrences of Theorem 6.1, taken in pairs. They are analogous to the contiguity relations (or 'contiguous function relations') of Gauss, for $F_{1}$, which follow by elimination from the differential recurrences of Jacobi, though Gauss did not derive them in this way.

The explicit formulas for the functions $r_{n}^{m}$ with small $n, m$ given in Sect.3.1 (see Eqs. (3.3),(3.4)) also follow from the differential recurrences of Theorem 6.1.

Theorem 6.2 For any $m \in \mathbb{Z}$, the octahedral function $r_{0}^{m}$ has the hypergeometric representation

$$
r_{0}^{m}(u)={ }_{2} F_{1}\left(-2 m,-\frac{1}{4}-3 m ; \frac{3}{4}-m \mid u\right) .
$$

Thus when $m \geq 0, r_{0}^{m}$ is a degree-2m hypergeometric polynomial. Moreover, for any $n, m \geq 0, r_{n}^{m}$ is a polynomial of degree $3 n+2 m$.

Proof When $n=0, \mathcal{L}_{n}^{m} f=0$ loses two singular points and degenerates to a Gauss hypergeometric equation of the form (6.1), with independent variable $u$ and parameters $a=-2 m, b=-\frac{1}{4}-3 m, c=\frac{3}{4}-m$. Hence $r_{0}^{m}(u)$ has the claimed representation, and if $m \geq 0$, is a degree- $2 m$ polynomial in $u$. It follows by induction from the differential recurrence with $\Delta(n, m)=(+1,0)$ that $r_{n}^{m}(u)$ is a polynomial in $u$ for all $n \geq 0$. It must be of degree $3 n+2 m$, because in the P-symbol of $\mathcal{L}_{n}^{m}$ [see (6.5b)], the only characteristic exponent at $u=\infty$ that is a (nonpositive) integer is $-2 m-3 n$.

The statement of this theorem includes additional claims that were made in Sect. 3.1. The following related theorem mentions the Heun function $\operatorname{Hn}(a, q ; \alpha, \beta, \gamma, \delta \mid z)$, for the definition of which see [34]. This is a Frobenius solution (at $z=0$ ) of a canonical Fuchsian differential equation that has four singular points, namely $z=0,1, a, \infty$, and an 'accessory' parameter $q$ that unlike $\alpha, \beta, \gamma, \delta$, does not affect their characteristic exponents. It has a convergent expansion $\sum_{k=0}^{\infty} h_{k} z^{k}$, where the $\left\{h_{k}\right\}_{k=0}^{\infty}$ satisfy a second-order recurrence with coefficients quadratic in $k$. 
Theorem 6.3 For any $n \in \mathbb{Z}$, the octahedral function $r_{n}^{0}$ has the Heun representation

$r_{n}^{0}(u)=H n\left(\left(\frac{2+\sqrt{3}}{2-\sqrt{3}}\right)^{2}, \frac{9(2+\sqrt{3})^{2} n(12 n+1)}{4} ;-3 n,-\frac{1}{4}-3 n ; \frac{3}{4},-3 n ;-(2+\sqrt{3})^{2} u\right)$

and the equivalent expansion $\sum_{k=0}^{\infty} a_{k} u^{k}$, where $\left\{a_{k}\right\}_{k=0}^{\infty}$ satisfy the second-order recurrence

$$
\begin{aligned}
& (k+1)(4 k+3) a_{k+1}+[14 k(4 k-12 n-1)+9 n(12 n+1)] a_{k} \\
& \quad+(k-3 n-1)(4 k-12 n-5) a_{k-1}=0,
\end{aligned}
$$

with $a_{0}=1, a_{-1}=0$. Thus when $n \geq 0, r_{n}^{0}$ is a degree- $3 n$ Heun polynomial.

Proof If $m=0$, the $u=1$ singular point of $\mathcal{L}_{n}^{m}$ drops out, i.e., becomes ordinary, and

$$
\mathcal{L}_{n}^{0}=D_{u}^{2}+\left[\frac{3 / 4}{u}+\frac{-3 n(2 u+14)}{u^{2}+14 u+1}\right] D_{u}+\frac{3 n(1+12 n)}{4} \frac{u+3}{u\left(u^{2}+14 u+1\right)} .
$$

The substitution $z=-(2+\sqrt{3})^{2} u$ reduces $\mathcal{L}_{n}^{0} f=0$ to the standard Heun equation [34], with the stated values of $a, q ; \alpha, \beta, \gamma, \delta$. The recurrence (6.8), based on a second-order difference operator, comes by substituting $f=r_{n}^{0}=\sum_{k=0}^{\infty} a_{k} u^{k}$ into $\mathcal{L}_{n}^{0} f=0$.

For general $(n, m) \in \mathbb{Z}^{2}, \mathcal{L}_{n}^{m} f=0$ has five singular points. The theory of such generalized Heun equations is underdeveloped at present, but the coefficients of their series solutions are known to satisfy third-order (i.e., four-term) recurrences.

Theorem 6.4 For any $(n, m) \in \mathbb{Z}^{2}$, the octahedral function $r_{n}^{m}$ has the expansion $r_{n}^{m}(u)=\sum_{k=0}^{\infty} a_{k} u^{k}$, where $\left\{a_{k}\right\}_{k=0}^{\infty}$ satisfy the third-order recurrence

$$
\begin{aligned}
(k+1) & (4 k-4 m+3) a_{k+1} \\
& +[k(52 k-36 m-168 n-13)-(2 m-9 n)(12 m+12 n+1)] a_{k} \\
& -[(k-1)(52 k-276 m-144 n-65)+2(14 m+3 n)(12 m+12 n+1)] a_{k-1} \\
& -(k-2 m-3 n-2)(4 k-12 m-12 n-9) a_{k-2}=0,
\end{aligned}
$$

with $a_{0}=1, a_{-1}=0, a_{-2}=0$. Thus when $n, m \geq 0, r_{n}^{m}(u)$ is a degree- $(3 n+2 m)$ generalized Heun polynomial.

Proof The recurrence comes by substituting $f=r_{n}^{m}=\sum_{k=0}^{\infty} a_{k} u^{k}$ into $\mathcal{L}_{n}^{m} f=0$.

It can be shown that if $m=0$, the third-order (i.e., generalized Heun) difference operator in (6.9) has the second-order (i.e., Heun) difference operator in (6.8) as a right factor, and if $n=0$, it has a first-order (i.e., hypergeometric) difference operator as a right factor, which is why the representation in Theorem 6.2 exists. The coefficients of all these difference operators are quadratic in $k$. 
As stated in Theorem 3.2, it is not merely the case that when $n, m \geq 0$, the rational function $r_{n}^{m}=r_{n}^{m}(u)$ is a polynomial of degree $3 n+2 m$. In each quadrant of the $(n, m)$-plane, it is the quotient of a polynomial of known degree (the numerator) by a known polynomial (the denominator). To obtain the formulas in Theorem 3.2 that refer to quadrants other than the first, reason as follows. Consider the second formula: it says that if $n, m \geq 0, r_{n}^{-m-1}(u)$ equals a polynomial of degree $1+3 n+2 m$, divided by $(1-u)^{3+4 m}$. This is proved by induction on $n$, the base case $(n=0)$ being

$$
r_{0}^{-m-1}(u)=(1-u)_{2}^{-3-4 m} F_{1}\left(-1-2 m,-\frac{1}{4}-m ; \frac{3}{4}-m \mid u\right),
$$

which comes from (6.7) by Euler's transformation of ${ }_{2} F_{1}$. The inductive step uses the differential recurrence with $\Delta(n, m)=(+1,0)$, as in the proof of Theorem 6.2. In the same way, the third and fourth formulas follow from the $\Delta(n, m)=(-1,0)$ recurrence.

One sees from the four formulas in Theorem 3.2 that irrespective of quadrant, $r_{n}^{m} \sim$ const $\times u^{3 n+2 m}$, which partially confirms the claims of Theorem 3.1(i,ii). A consequence of this asymptotic behavior is that besides being the Frobenius solution associated with the exponent 0 at $u=0, r_{n}^{m}$ is the Frobenius solution associated with the exponent $-2 m-3 n$ at $u=\infty$, which appeared in the P-symbol (6.5b).

Theorem 3.1 states specifically that $r_{n}^{m} \sim d_{n}^{m} \times u^{3 n+2 m}$, with $d_{n}^{m}$ defined in (3.2). This too is proved by induction. The base case $(n=0)$ has sub-cases $m \geq 0$ and $m \leq 0$, which follow by elementary manipulations from (6.7) and (6.10), respectively. The inductions toward $n \geq 0$ and $n \leq 0$ come from the differential recurrences with $\Delta(n, m)=( \pm 1,0)$, the $u \rightarrow \infty$ asymptotics of which yield expressions for $d_{n \pm 1}^{m} / d_{n}^{m}$. As one can check, these two expression agree with what (3.2) predicts.

The only claim in Sect.3.1 remaining to be proved is Theorem 3.1(iii): the statement that the conjugate function $\bar{r}_{n}^{m}=\bar{r}_{n}^{m}(u)$ is related to $r_{n}^{m}=r_{n}^{m}(u)$ by $\bar{r}_{n}^{m}(u) \propto u^{3 n+2 m} r_{n}^{m}(1 / u)$, or equivalently $r_{n}^{m}(u) \propto u^{3 n+2 m} \bar{r}_{n}^{m}(1 / u)$. (The constant of proportionality comes from $\bar{r}_{n}^{m}(0)$ equalling unity.)

Just as one derives the differential equation $\mathcal{L}_{n}^{m} r_{n}^{m}=0$ of Theorem 6.1 by 'lifting and shifting,' one can derive an equation $\overline{\mathcal{L}}_{n}^{m} \bar{r}_{n}^{m}=0$ on the $u$-sphere from the definition of $\bar{r}_{n}^{m}$ given in Definition 3.1, and a further equation satisfied by $\mathbf{r}_{n}^{m}(u):=$ $u^{3 n+2 m} \bar{r}_{n}^{m}(1 / u)$. The latter turns out to be $\mathcal{L}_{n}^{m} \mathbf{r}_{n}^{m}=0$, i.e., to be identical to the equation of Theorem 6.1. But $\bar{r}_{n}^{m}=\bar{r}_{n}^{m}(u)$, analytic at $u=0$, is the Frobenius solution associated with the exponent 0 at $u=0$ of $\overline{\mathcal{L}}_{n}^{m} \bar{r}_{n}^{m}=0$. Hence, $\mathbf{r}_{n}^{m}$ is the Frobenius solution associated with the exponent $-2 m-3 n$ at $u=\infty$ of $\mathcal{L}_{n}^{m} \mathbf{r}_{n}^{m}=0$. But as was noted three paragraphs ago, this is $r_{n}^{m}$; so $\mathbf{r}_{n}^{m} \propto r_{n}^{m}$, and Theorem 3.1(iii) follows.

\section{Biorthogonality of Octahedral Functions}

The octahedral functions $r_{n}^{m}=r_{n}^{m}(u)$, which are polynomials if $n, m \geq 0$, satisfy recurrences, such as the three-term ones of Theorem 3.3, that are quite unlike the ones satisfied by the classical orthogonal polynomials. But at least if $m=0,-1$, it can be shown that the family $\left\{r_{n}^{m}\right\}_{m \in \mathbb{Z}}$ displays orthogonality on the $u$-interval $[0,1]$, or rather a form of biorthogonality. 
The biorthogonality is best expressed in terms of the lifted functions $\tilde{r}_{n}^{m}(s):=$ $r_{n}^{m}\left(u=s^{4}\right)$ of the last section, the full domain of which is the Riemann $s$-sphere in which the defining octahedron is inscribed. These are solutions of $\tilde{\mathcal{L}}_{n}^{m} \tilde{f}=0$, where the operator $\tilde{\mathcal{L}}_{n}^{m}$ was defined in (6.4). By inspection, it has the simpler representation

$$
\tilde{\mathcal{L}}_{n}^{m}=\left(q_{\mathrm{v}}^{2 m} q_{\mathrm{f}}^{3 n / 2}\right) D_{s}^{2}\left(q_{\mathrm{v}}^{2 m} q_{\mathrm{f}}^{3 n / 2}\right)^{-1}+\left\{[-2 m(1+2 m)] \frac{q_{\mathrm{f}}}{q_{\mathrm{v}}^{2}}+[144 n(2+3 n)] \frac{q_{\mathrm{v}}^{2}}{q_{\mathrm{f}}^{2}}\right\}
$$

where $q_{\mathrm{v}}(s)=s\left(1-s^{4}\right)$ and $q_{\mathrm{f}}(s)=1+14 s^{4}+s^{8}$ are the usual polynomials that equal zero at the five finite vertices and the eight face-centers of the octahedron.

By (7.1), $\tilde{\mathcal{L}}_{n}^{m}$ is conjugated by a similarity transformation to a formally self-adjoint operator of the Schrödinger type. For any fixed $m$, the calculation of the eigenfunctions $q_{\mathrm{v}}^{-2 m} q_{\mathrm{f}}^{-3 n / 2} \tilde{r}_{n}^{m}(s)$ of the latter, on the $s$-interval $[0,1]$, can be viewed as solving a Sturm-Liouville problem. The coefficient $144 n(2+3 n)$ in (7.1) plays the role of the Sturm-Liouville eigenvalue, and $q_{\mathrm{v}}^{2} / q_{\mathrm{f}}^{2}$ that of the Sturm-Liouville weight function.

Because the coefficient function $q_{\mathrm{f}} / q_{\mathrm{v}}^{2}$ diverges at the endpoints $s=0,1$, this Sturm-Liouville problem is typically a singular one. To avoid a discussion of endpoint classifications and boundary conditions, it is best to derive orthogonality results not from $\tilde{\mathcal{L}}_{n}^{m}$, but rather from the Love-Hunter biorthogonality relation (1.1); i.e.,

$$
\int_{-1}^{1} \mathrm{P}_{v}^{\mu}(z) \mathrm{P}_{v^{\prime}}^{-\mu}(-z) \mathrm{d} z=0
$$

which holds if $\mu \in(-1,1)$ and $\nu, v^{\prime}$ differ by a nonzero even integer. (See [21, Appendix] for a proof.) Equation (7.2) is a relation of orthogonality between the eigenfunctions of a singular boundary value problem based on (2.1), the associated Legendre equation (i.e., $\mathrm{P}_{\nu_{0}+2 n}^{\mu}(z), n \in \mathbb{Z}$ ), and the eigenfunctions of the adjoint boundary value problem (i.e., $\mathrm{P}_{v_{0}+2 n}^{-\mu}(-z), n \in \mathbb{Z}$ ). The first problem is non-self-adjoint because the boundary conditions that single out $\mathrm{P}_{\nu_{0}+2 n}^{\mu}(z), n \in \mathbb{Z}$, as eigenfunctions are not selfadjoint.

However, one feature of the operator $\tilde{\mathcal{L}}_{n}^{m}$ must be mentioned. If $\tilde{f}=\tilde{f}(s)$ solves $\tilde{\mathcal{L}}_{n}^{m} \tilde{f}=0$, then so does $(1-s)^{1+12 m+12 n} \tilde{f}((1+s) /(1-s))$. This claim can be verified by a lengthy computation, but its correctness is indicated by the P-symbol of $\tilde{\mathcal{L}}_{n}^{m}$, which appeared in $(6.3 \mathrm{c})$. The map $s \mapsto(1+s) /(1-s)$ is a $90^{\circ}$ rotation of the $s$-sphere, and hence of the inscribed octahedron, around the axis through the equatorial vertices $s= \pm \mathrm{i}$. This rotation takes vertices to vertices, edges to edges, and faces to faces. The subsequent multiplication by $(1-s)^{1+12 m+12 n}$ shifts the characteristic exponents at the most affected vertices $(s=1, \infty)$ to the values they had before the rotation.

Theorem 7.1 For $m=0$ and $m=-1$, the lifted family $\left\{\tilde{r}_{n}^{m}(s):=r_{n}^{m}\left(s^{4}\right)\right\}_{n \in \mathbb{Z}}$ is biorthogonal on the s-interval $[0,1]$ in the following sense: the inner product integral 


$$
\begin{aligned}
\int_{0}^{1} & {\left[q_{\mathrm{v}}^{-2 m} q_{\mathrm{f}}^{-3 n / 2}(s) \cdot \tilde{r}_{n}^{m}(s)\right] } \\
& \times\left[q_{\mathrm{v}}^{-2 m} q_{\mathrm{f}}^{-3 n^{\prime} / 2}(s) \cdot(1-s)^{1+12 m+12 n} \tilde{r}_{n^{\prime}}^{m}\left(\frac{1+s}{1-s}\right)\right] \frac{q_{\mathrm{v}}^{2}}{q_{\mathrm{f}}^{2}}(s) \mathrm{d} s
\end{aligned}
$$

equals zero if $n, n^{\prime}$ differ by a nonzero even integer.

Proof Substitute the $m=0,-1$ cases of the formulas for $\mathrm{P}_{-\frac{1}{6}+n}^{ \pm\left(\frac{1}{4}+m\right)}(\cos \theta)$ in Theorem 3.5 into (7.2) and change the variable of integration from $z=\cos \theta$ to $u=B_{-} / B_{+}$ and then to $s=u^{1 / 4}$. The involution $z \mapsto-z$ corresponds to $s \mapsto(1-s) /(1+s)$.

This biorthogonality theorem is formulated so as to indicate its close connection to Sturm-Liouville theory: evaluating the integral over $0<s<1$ computes the inner product of the two square-bracketed factors in the integrand, which come from $\mathrm{P}_{v}^{\mu}(z)$ and $\mathrm{P}_{v^{\prime}}^{-\mu}(-z)$, with respect to the weight function $q_{\mathrm{v}}^{2} / q_{\mathrm{f}}^{2}$. The two factors are eigenfunctions of adjoint Sturm-Liouville problems on $0<s<1$ (i.e., ones with adjoint boundary conditions), with different eigenvalues.

Theorem 7.1 cannot be extended to general $m \in \mathbb{Z}$, because the integral diverges unless $m=0$ or $m=-1$, owing to rapid growth of one or the other of the bracketed factors at each of the endpoints $s=0,1$. This divergence follows readily from the results on $r_{n}^{m}$ given in Theorems 3.1 and 3.2. Alternatively, the divergence arises from the Ferrers function $\mathrm{P}_{v}^{\mu}$ not lying in $L^{2}[-1,1]$ when $\mu$ is nonintegral, unless $\operatorname{Re} \mu \in(-1,1)$.

The formulas for the tetrahedral Ferrers functions $\mathrm{P}_{-\frac{3}{4}-m}^{ \pm\left(\frac{1}{3}+n\right)}$ given in Theorem 4.3 (cases $m=0,-1$ ) can also be substituted usefully into the Love-Hunter relation (7.2). But the resulting statement of biorthogonality is more complicated than Theorem 7.1 and is not given here.

\section{Cyclic and Dihedral Formulas (Schwarz Classes O and I)}

This section derives parametric formulas for Legendre and Ferrers functions that are cyclic or dihedral. The formulas involve the Jacobi polynomials $P_{n}^{(\alpha, \beta)}$ and are unrelated to the octahedral and tetrahedral ones in Sects. 3, 4, and 5. They are of independent interest, and subsume formulas that have previously appeared in the literature.

As used here, 'cyclic' and 'dihedral' have extended meanings. The terms arise as follows. The associated Legendre equation (2.1) has $(\mu, \mu, 2 v+1)$ as its (unordered, unsigned) triple of characteristic exponent differences. By the results of Schwarz on the algebraicity of hypergeometric functions, this differential equation will have only algebraic solutions if $\left(v+\frac{1}{2}, \mu\right)$ lies in $\left( \pm \frac{1}{2}, \pm \frac{1}{2 k}\right)+\mathbb{Z}^{2}$ or $\left( \pm \frac{1}{2 k}, \pm \frac{1}{2}\right)+\mathbb{Z}^{2}$, for some positive integer $k$. These restrictions cause the equation to lie in Schwarz's cyclic class (labelled O here), resp. his dihedral class I. The terms refer to the projective monodromy group of the equation, which is a (finite) subgroup of $P S L(2, \mathbb{R})$.

However, the formulas derived below are more general, in that they allow $k$ to be arbitrary: they are formulas for continuously parametrized families of Legendre and 
Ferrers functions, which are generically transcendental rather than algebraic. Because of this, we call a Legendre or Ferrers function cyclic, resp. dihedral, if $\left(v+\frac{1}{2}, \mu\right)$ lies in $\left( \pm \frac{1}{2}, *\right)+\mathbb{Z}^{2}$, resp. $\left(*, \pm \frac{1}{2}\right)+\mathbb{Z}^{2}$, the asterisk denoting an unspecified value. That is, the degree $v$ should be an integer or the order $\mu$ a half-odd-integer, respectively.

Explicit formulas in terms of Jacobi polynomials are derived in Sect. 8.1, and how dihedral Ferrers functions can be used for expansion purposes is explained in Sect. 8.2.

\subsection{Explicit Formulas}

The Jacobi polynomials $P_{n}^{(\alpha, \beta)}(z)$ are well known [11, Sect. 10.8]. They have the hypergeometric and Rodrigues representations

$$
\begin{aligned}
P_{n}^{(\alpha, \beta)}(z) & =\frac{(\alpha+1)_{n}}{n !}{ }_{2} F_{1}\left(\begin{array}{c|c}
-n, n+\alpha+\beta+1 \\
\alpha+1
\end{array} \mid \frac{1-z}{2}\right) \\
& =\frac{(-1)^{n}}{2^{n} n !}(1-z)^{-\alpha}(1+z)^{-\beta} \frac{\mathrm{d}^{n}}{\mathrm{~d} z^{n}}\left[(1-z)^{\alpha+n}(1+z)^{\beta+n}\right]
\end{aligned}
$$

and are orthogonal on $[-1,1]$ with respect to the weight function $(1-x)^{\alpha}(1+x)^{\beta}$, if $\alpha, \beta>-1$ and the weight function is integrable.

Legendre and Ferrers functions that are cyclic (i.e., of integer degree) are easily expressed in terms of Jacobi polynomials.

Theorem 8.1 The formulas

$$
\begin{aligned}
& P_{-\frac{1}{2} \pm\left(n+\frac{1}{2}\right)}^{\mu}(z)=\frac{n !}{\Gamma(n-\mu+1)}\left(\frac{z+1}{z-1}\right)^{\mu / 2} P_{n}^{(-\mu, \mu)}(z), \\
& P_{-\frac{1}{2} \pm\left(n+\frac{1}{2}\right)}^{\mu}(\cosh \xi)=\frac{n !}{\Gamma(n-\mu+1)}[\operatorname{coth}(\xi / 2)]^{\mu} P_{n}^{(-\mu, \mu)}(\cosh \xi)
\end{aligned}
$$

hold when $n$ is a nonnegative integer, for $z \in(1, \infty)$ and $\xi \in(0, \infty)$. (In the degenerate case when $\mu-n$ is a positive integer, $P_{-\frac{1}{2} \pm\left(n+\frac{1}{2}\right)}^{\mu} \equiv 0$.)

Proof Compare the representations (2.8a) and (8.1a).

Theorem 8.2 The formulas

$$
\begin{aligned}
& \mathrm{P}_{-\frac{1}{2} \pm\left(n+\frac{1}{2}\right)}^{\mu}(z)=\frac{n !}{\Gamma(n-\mu+1)}\left(\frac{1+z}{1-z}\right)^{\mu / 2} P_{n}^{(-\mu, \mu)}(z), \\
& \mathrm{P}_{-\frac{1}{2} \pm\left(n+\frac{1}{2}\right)}^{\mu}(\cos \theta)=\frac{n !}{\Gamma(n-\mu+1)}[\cot (\theta / 2)]^{\mu} P_{n}^{(-\mu, \mu)}(\cos \theta)
\end{aligned}
$$

hold when $n$ is a nonnegative integer, for $z \in(-1,1), \xi \in(-\infty, \infty)$, and $\theta \in(0, \pi)$. (In the degenerate case when $\mu-n$ is a positive integer, $\mathrm{P}_{-\frac{1}{2} \pm\left(n+\frac{1}{2}\right)}^{\mu} \equiv 0$.) 
Proof The proof is accomplished by analytic continuation of Theorem 8.1, or in effect, by letting $\xi=\mathrm{i} \theta$.

By exploiting the $\hat{Q} \rightarrow P$ and $\mathrm{Q} \rightarrow$ P reductions (2.6) and (2.7), one can derive additional formulas from Theorems 8.1 and 8.2, for $\hat{Q}_{-\frac{1}{2} \pm\left(n+\frac{1}{2}\right)}^{\mu}$ and $\mathrm{Q}_{-\frac{1}{2} \pm\left(n+\frac{1}{2}\right)}^{\mu}$ respectively. However, the coefficients in (2.6) and (2.7) diverge when $\mu \in \mathbb{Z}$. Hence, following this approach to formulas for $\hat{Q}_{-\frac{1}{2} \pm\left(n+\frac{1}{2}\right)}^{m}, \mathrm{Q}_{-\frac{1}{2} \pm\left(n+\frac{1}{2}\right)}^{m}$, when $n$ is a nonnegative integer and $m$ an integer, requires the taking of a limit. In the commonly encountered case when $-n \leq m \leq n$ (but not otherwise), the resulting expressions turn out to be logarithmic. Such expressions can be computed in other ways [11, Sect. 3.6.1]. Perhaps the best method is to express $\hat{Q}_{-\frac{1}{2} \pm\left(n+\frac{1}{2}\right)}^{m}$ in terms of a ${ }_{2} F_{1}$ by using (2.8d), and then use known formulas for logarithmic ${ }_{2} F_{1}$ 's [5].

Legendre and Ferrers functions that are dihedral (i.e., are of half-odd-integer order) are the subject of the following theorems. For conciseness, a special notation is used: $[A \mid B]_{ \pm}$signifies $A$, resp. $B$, in the + , resp. - case, and $\{C\}_{\alpha, \pm}$, where $C$ depends on $\alpha$, signifies the even or odd part of $C$ under $\alpha \mapsto-\alpha$, i.e., $\frac{1}{2}[C(\alpha) \pm C(-\alpha)]$.

Theorem 8.3 The formulas

$$
\begin{aligned}
\hat{Q}_{-\frac{1}{2}+\alpha}^{ \pm\left(\frac{1}{2}+m\right)}(z)= & \sqrt{\frac{\pi}{2}} m !\left[1 \mid \frac{1}{(\alpha-m)_{2 m+1}}\right]_{ \pm} \\
& \times\left(z^{2}-1\right)^{-1 / 4}\left(z+\sqrt{z^{2}-1}\right)^{-\alpha} P_{m}^{(\alpha,-\alpha)}\left(\frac{z}{\sqrt{z^{2}-1}}\right) \\
\hat{Q}_{-\frac{1}{2}+\alpha}^{ \pm\left(\frac{1}{2}+m\right)}(\cosh \xi) & =\sqrt{\frac{\pi}{2}} m !\left[1 \mid \frac{1}{(\alpha-m)_{2 m+1}}\right]_{ \pm} \\
& \times(\sinh \xi)^{-1 / 2} \mathrm{e}^{-\alpha \xi} P_{m}^{(\alpha,-\alpha)}(\operatorname{coth} \xi)
\end{aligned}
$$

hold when $m$ is a nonnegative integer, for $z \in(1, \infty)$ and $\xi \in(0, \infty)$.

Proof Combine Whipple's $\hat{Q} \rightarrow P$ transformation [11, 3.3(13)], which appeared as Eq. (4.1), with the results in Theorem 8.1, and write $m$ for $n$, and $-\alpha$ for $\mu$.

In these formulas, the proportionality of $\hat{Q}_{-\frac{1}{2}+\alpha}^{ \pm\left(\frac{1}{2}+m\right)}$ to each other is expected; cf. (2.4). Also, the division in the 'minus' case by

$$
(\alpha-m)_{2 m+1}=(\alpha-m) \ldots(\alpha+m),
$$

which equals zero if $\alpha=-m, \ldots, m$, is not unexpected. As was noted in Sect. $2, \hat{Q}_{\nu}^{\mu}$ is undefined if $v+\mu$ is a negative integer, except when $v=-\frac{3}{2},-\frac{5}{2}, \ldots$, in which case $\hat{Q}_{v}^{v+1}, \ldots, \hat{Q}_{\nu}^{-(\nu+1)}$ are defined. This implies that for $m=0,1,2, \ldots, \hat{Q}_{-\frac{1}{2}+\alpha}^{+\left(\frac{1}{2}+m\right)}$ is defined for all $\alpha$, and that $\hat{Q}_{-\frac{1}{2}+\alpha}^{-\left(\frac{1}{2}+m\right)}$ is undefined if and only if $\alpha=-m, \ldots, m$. 
Theorem 8.4 The formulas

$$
\begin{aligned}
P_{-\frac{1}{2}+\alpha}^{ \pm\left(\frac{1}{2}+m\right)}(z)= & \sqrt{\frac{2}{\pi}} m !\left[(-1)^{m} \mid \frac{(-1)^{m+1}}{(\alpha-m)_{2 m+1}}\right]_{ \pm} \\
& \times\left(z^{2}-1\right)^{-1 / 4}\left\{\left(z+\sqrt{z^{2}-1}\right)^{-\alpha} P_{m}^{(\alpha,-\alpha)}\left(\frac{z}{\sqrt{z^{2}-1}}\right)\right\}_{\alpha, \pm} \\
P_{-\frac{1}{2}+\alpha}^{ \pm\left(\frac{1}{2}+m\right)}(\cosh \xi) & =\sqrt{\frac{2}{\pi}} m !\left[(-1)^{m} \mid \frac{(-1)^{m+1}}{(\alpha-m)_{2 m+1}}\right]_{ \pm} \\
& \times(\sinh \xi)^{-1 / 2}\left\{\mathrm{e}^{-\alpha \xi} P_{m}^{(\alpha,-\alpha)}(\operatorname{coth} \xi)\right\}_{\alpha, \pm}
\end{aligned}
$$

hold when $m$ is a nonnegative integer, for $z \in(1, \infty)$, and $\xi \in(0, \infty)$, it being understood in the 'minus' case that when $\alpha=-m, \ldots, m$ and there is an apparent division by zero, each right-hand side requires the taking of a limit.

Proof Combine the $P \rightarrow \hat{Q}$ reduction (2.5) with the results in Theorem 8.3.

Theorem 8.5 The formulas

$$
\begin{aligned}
& \mathrm{P}_{-\frac{1}{2}+\alpha}^{ \pm\left(\frac{1}{2}+m\right)}(\cos \theta)= \sqrt{\frac{2}{\pi}} m !\left[\mathrm{i}^{m} \mid \frac{\mathrm{i}^{-m-1}}{(\alpha-m)_{2 m+1}}\right]_{ \pm} \\
& \times(\sin \theta)^{-1 / 2}\left\{\mathrm{e}^{\mathrm{i} \alpha \theta} P_{m}^{(\alpha,-\alpha)}(\mathrm{i} \cot \theta)\right\}_{\alpha, \pm}, \\
& \mathrm{Q}_{-\frac{1}{2}+\alpha}^{ \pm\left(\frac{1}{2}+m\right)}(\cos \theta)=\sqrt{\frac{\pi}{2}} m !\left[\mathrm{i}^{m+1} \mid \frac{\mathrm{i}^{-m}}{(\alpha-m)_{2 m+1}}\right]_{ \pm} \\
& \times(\sin \theta)^{-1 / 2}\left\{\mathrm{e}^{\mathrm{i} \alpha \theta} P_{m}^{(\alpha,-\alpha)}(\mathrm{i} \cot \theta)\right\}_{\alpha, \mp}
\end{aligned}
$$

hold when $m$ is a nonnegative integer, for $\theta \in(0, \pi)$. In the sub-cases $\alpha=-m, \ldots, m$ of the 'minus' case, the apparent division by zero in the first formula is handled by interpreting its right-hand side in a limiting sense, but the division by zero in the second formula causes both its sides to be undefined.

Proof The first formula follows by analytic continuation of the latter formula in Theorem 8.4 , in effect, by letting $\xi=-\mathrm{i} \theta$. The second formula then follows from the $\mathrm{Q} \rightarrow \mathrm{P}$ reduction (2.7), after some algebraic manipulations.

As was noted in Sect. 2, $Q_{v}^{\mu}$ is undefined if and only if $\hat{Q}_{v}^{\mu}$ is. It was also noted that if $\mu=\frac{1}{2}, \frac{3}{2}, \ldots$, then $\mathrm{Q}_{-\mu}^{\mu}, \ldots, \mathrm{Q}_{\mu-1}^{\mu} \equiv 0$. It follows that in the 'plus' case of the second formula of the theorem, the right-hand side must equal zero if $\alpha=-m, \ldots, m$. This yields the interesting Jacobi-polynomial identity

$$
\mathrm{e}^{\mathrm{i} \alpha \theta} P_{m}^{(\alpha,-\alpha)}(\mathrm{i} \cot \theta)=\mathrm{e}^{-\mathrm{i} \alpha \theta} P_{m}^{(-\alpha, \alpha)}(\mathrm{i} \cot \theta),
$$

which holds for $m=0,1,2, \ldots$, when $\alpha=0,1, \ldots, m$. 


\subsection{Dihedral Ferrers Functions and Love-Hunter Expansions}

In this subsection, we show that an expansion in dihedral Ferrers functions can be, in effect, an expansion in Chebyshev polynomials (of the fourth kind), and as an application, we show that the result of [32] on the convergence of Love-Hunter expansions can be slightly extended.

The first formula on dihedral Ferrers functions in Theorem 8.5 specializes when $m=0$ to the known pair of formulas [31, Sect. 14.5]

$$
\mathrm{P}_{-\frac{1}{2}+\alpha}^{-\frac{1}{2}}(\cos \theta)=\sqrt{\frac{2}{\pi}} \frac{\sin (\alpha \theta)}{\alpha \sqrt{\sin \theta}}, \quad \mathrm{P}_{-\frac{1}{2}+\alpha}^{\frac{1}{2}}(\cos \theta)=\sqrt{\frac{2}{\pi}} \frac{\cos (\alpha \theta)}{\sqrt{\sin \theta}} .
$$

These hold for $\theta \in(0, \pi)$, the $\alpha=0$ case of the former requiring the taking of a limit.

Love-Hunter biorthogonality, i.e., the orthogonality of the functions $\mathrm{P}_{v}^{\mu}(z)$ and $\mathrm{P}_{\nu}^{-\mu}(-z)$ in $L^{2}[-1,1]$ when (i) $\operatorname{Re} \mu \in(-1,1)$ and (ii) $v, v^{\prime}$ differ by an even integer and are not half-odd-integers, specializes when $\mu=-\frac{1}{2}$ and $z=\cos \theta$ to

$$
\int_{0}^{\pi} \mathrm{P}_{v}^{-\frac{1}{2}}(\cos \theta) \mathrm{P}_{v^{\prime}}^{\frac{1}{2}}(-\cos \theta) \sin \theta \mathrm{d} \theta=0
$$

and thus to

$$
\int_{0}^{\pi} \sin (\alpha \theta) \cos \left[\alpha^{\prime}(\pi-\theta)\right] d \theta=0,
$$

which holds if $\alpha, \alpha^{\prime}$ differ by an even integer. (By continuity, the restriction to $\alpha, \alpha^{\prime}$ that are not integers can be dropped.) The orthogonality in (8.3) is not well known.

A Love-Hunter expansion of an 'arbitrary' function $f=f(z)$ on $-1<z<1$ is a bilateral expansion of $f$ in the Ferrers functions $\mathrm{P}_{v_{0}+2 n}^{\mu}$, of the form (1.2), in which the coefficients $\left\{c_{n}\right\}_{n \in \mathbb{Z}}$ are computed as inner products; i.e.,

$$
c_{n}^{(\alpha)}=\frac{\int_{-1}^{1} \mathrm{P}_{v_{0}+2 n}^{-\mu}(-z) f(z) \mathrm{d} z}{\int_{-1}^{1} \mathrm{P}_{\nu_{0}+2 n}^{-\mu}(-z) \mathrm{P}_{\nu_{0}+2 n}^{\mu}(z) \mathrm{d} z} .
$$

Existing results on the convergence of such expansions [20,21] require that $|\operatorname{Re} \mu|<\frac{1}{2}$, or in the real case, $\mu \in\left(-\frac{1}{2}, \frac{1}{2}\right)$.

It is of interest to examine whether convergence results can also be obtained in the boundary cases $\mu= \pm \frac{1}{2}$. To treat the case when $\left(v_{0}, \mu\right)=\left(-\frac{1}{2}+\alpha,-\frac{1}{2}\right)$, define the indexed $(n \in \mathbb{Z})$ and continuously parametrized $(\alpha \in \mathbb{R})$ functions

$$
\psi_{n}^{(\alpha)}(\theta)=\frac{\sin [(2 n+\alpha) \theta]}{\sqrt{\sin \theta}}, \quad \chi_{n}^{(\alpha)}(\theta)=\frac{\cos [(2 n+\alpha)(\pi-\theta)]}{\sqrt{\sin \theta}}
$$

on $0<\theta<\pi$, which are biorthogonal with respect to the weight function $\sin \theta$. (They differ only in normalization from $\mathrm{P}_{-\frac{1}{2}+\alpha+2 n}^{-\frac{1}{2}}$ and $\mathrm{P}_{-\frac{1}{2}+\alpha+2 n}^{\frac{1}{2}}$.) In terms of the first, one has a formal $\mu=-\frac{1}{2}$ Love-Hunter expansion 


$$
f(\cos \theta)=\sum_{n=-\infty}^{\infty} c_{n}^{(\alpha)} \psi_{n}^{(\alpha)}(\theta)
$$

where

$$
c_{n}^{(\alpha)}=\frac{\int_{0}^{\pi} \chi_{n}^{(\alpha)}(\theta) f(\cos (\theta)) \sin \theta \mathrm{d} \theta}{\int_{0}^{\pi} \chi_{n}^{(\alpha)}(\theta) \psi_{n}^{(\alpha)}(\theta) \sin \theta \mathrm{d} \theta} .
$$

The denominator inner product equals $(\pi / 2) \sin (\alpha \pi)$ for all $n$, by examination; hence the restriction $\alpha \in \mathbb{R} \backslash \mathbb{Z}$ must obviously be imposed.

This expansion is not fully satisfactory, because each $\psi_{n}^{(\alpha)}(\theta)$ diverges as $\theta \rightarrow \pi^{-}$, though it converges to zero, asymmetrically, as $\theta \rightarrow 0^{+}$. The underlying problem is that if $\operatorname{Re} \mu<0$, the function $\mathrm{P}_{v}^{\mu}(z)$ has leading behavior as $z \rightarrow 1^{-}$proportional to $(1-z)^{-\mu / 2}$, but its leading behavior as $z \rightarrow(-1)^{+}$comprises two terms: one proportional to $(1+z)^{-\mu / 2}$, and one to $(1+z)^{+\mu / 2}$.

In order (i) to make endpoint behavior more symmetrical and less divergent, and (ii) to study endpoint convergence, Pinsky [32] has proposed modifying Love-Hunter expansions by treating $[(1-z) /(1+z)]^{\mu / 2} \mathrm{P}_{v}^{\mu}(z)$ rather than $\mathrm{P}_{v}^{\mu}(z)$ as the expansion function. By (2.8a), this amounts to replacing each $\mathrm{P}_{v}^{\mu}(z)$ by the ${ }_{2} F_{1}$ function in terms of which it is defined, i.e., performing a hypergeometric expansion.

Adopting the suggestion of [32] when $\mu=-\frac{1}{2}$ amounts to replacing $\psi_{n}^{(\alpha)}, \chi_{n}^{(\alpha)}$ by versions that are multiplied by $[(1-z) /(1+z)]^{-1 / 4}$, which equals $\cot ^{1 / 2}(\theta / 2)$. With a trivial change in normalization, these are the functions

$$
\hat{\psi}_{n}^{(\alpha)}(\theta)=\frac{\sin [(2 n+\alpha) \theta]}{\sin (\theta / 2)}, \quad \hat{\chi}_{n}^{(\alpha)}(\theta)=\frac{\cos [(2 n+\alpha)(\pi-\theta)]}{\sin (\theta / 2)}
$$

( $n \in \mathbb{Z}$ ) on $0<\theta<\pi$, which are biorthogonal with respect to the weight function $\sin ^{2}(\theta / 2)$. Each $\hat{\psi}_{n}^{(\alpha)}(\theta)$ has a finite, nonzero limit as $\theta \rightarrow 0^{+}$and $\theta \rightarrow \pi^{-}$, and as a function of $z=\cos \theta$ is proportional to

$$
{ }_{2} F_{1}\left(\frac{1}{2}-\alpha-2 n, \frac{1}{2}+\alpha+2 n ; \frac{3}{2} ; \frac{1-z}{2}\right) \text {. }
$$

In terms of these trigonometric functions $\hat{\psi}_{n}^{(\alpha)}(\theta)$, one has (formally) a bilateral expansion of an arbitrary function $f=f(z)$ defined on $-1<z<1$; namely,

$$
f(z=\cos \theta)=\lim _{N \rightarrow \infty} \sum_{n=-N}^{N} \hat{c}_{n}^{(\alpha)} \hat{\psi}_{n}^{(\alpha)}(\theta)
$$

for all $\theta \in(0, \pi)$, where

$$
\hat{c}_{n}^{(\alpha)}=\frac{\int_{0}^{\pi} \hat{\chi}_{n}^{(\alpha)}(\theta) f(\cos (\theta)) \sin ^{2}(\theta / 2) \mathrm{d} \theta}{\int_{0}^{\pi} \hat{\chi}_{n}^{(\alpha)}(\theta) \hat{\psi}_{n}^{(\alpha)}(\theta) \sin ^{2}(\theta / 2) \mathrm{d} \theta} .
$$


The denominator in (8.6) equals $(\pi / 2) \sin (\alpha \pi)$ for all $n$, as before. One can clearly restrict $\alpha$ from $\mathbb{R} \backslash \mathbb{Z}$ to the interval $(0,1)$ without losing generality.

Theorem 8.6 If $f=f(z)$ is piecewise continuous on $-1 \leq z \leq 1$, then in the symmetric case $\alpha=\frac{1}{2}$, the bilateral series in (8.5) will converge as $N \rightarrow \infty$ to $f(z)$ at all points of continuity, including the endpoints, and in general to $[f(z+)+f(z-)] / 2$.

Proof The Chebyshev polynomials $W_{j}$ of the fourth kind, for $j=0,1,2, \ldots$, are defined by [24]

$$
W_{j}(\cos \theta)=\frac{\sin \left[\left(j+\frac{1}{2}\right) \theta\right]}{\sin \left(\frac{1}{2} \theta\right)}=\sum_{m=-j}^{j} \mathrm{e}^{\mathrm{i} m \theta} .
$$

It follows from (8.4) that when $n=0,1,2, \ldots$, both of $\hat{\psi}_{n}^{\left(\frac{1}{2}\right)}(\theta), \hat{\chi}_{n}^{\left(\frac{1}{2}\right)}(\theta)$ equal $W_{2 n}(\cos \theta)$, and when $n=-1,-2, \ldots$, they equal $-W_{-2 n-1}(\cos \theta)$. The bilateral expansion in (8.5) thus reduces if $\alpha=\frac{1}{2}$ to a unilateral expansion in the polynomials $W_{j}, j=0,1,2, \ldots$.

The Chebyshev polynomials $T_{k}$ of the first kind, for $k=0,1,2, \ldots$, are given by

$$
T_{k}(\cos \theta)=\cos (k \theta)
$$

By standard Fourier series theory, the expansion of $g=g(u)$ in the $T_{k}(u)$, when $g$ is piecewise continuous on $-1 \leq u \leq 1$, will converge to $g$ at all points of continuity, and in general to $[g(u+)+g(u-)] / 2$. But (see [24, Sect. 5.8.2]), if one writes $z=1-2 u^{2}$ (so that $u=\sin (\theta / 2)$ if $z=\cos \theta$ ), then $W_{j}(z)$ equals $(-1)^{j} u^{-1} T_{2 j+1}(u)$. Therefore an expansion of $f=f(z)$ in the fourth-kind $W_{j}(z)$ on $-1 \leq z \leq 1$ is effectively an expansion of $g(u)=u f\left(1-2 u^{2}\right)$ on $-1 \leq u \leq 1$ in the first-kind $T_{k}(u)$, each even- $k$ term of which must vanish. The theorem follows.

It is useful to compare this convergence result, which refers to an expansion of $f$ in the Ferrers functions $\mathrm{P}_{2 n}^{-\frac{1}{2}}$, with the pointwise convergence result of [32]. The latter deals with an expansion in the functions $\mathrm{P}_{\nu_{0}+2 n}^{\mu}$, where $\nu_{0}$ is arbitrary and $\mu \in\left(-\frac{1}{2}, \frac{1}{2}\right)$. However, it requires that $f$ be piecewise smooth, not merely piecewise continuous.

As the above theorem reveals, this assumption can be relaxed, at least in the seemingly difficult 'corner' case when $\left(v_{0}, \mu\right)=\left(0,-\frac{1}{2}\right)$. Whether smoothness can also be dropped as a hypothesis for the pointwise convergence of Love-Hunter expansions with $\mu \in\left(-\frac{1}{2}, \frac{1}{2}\right)$, or with $\left(v_{0}, \mu\right)=\left(-\frac{1}{2}+\alpha,-\frac{1}{2}\right)$ when $\alpha \neq \frac{1}{2}$, remains to be explored.

It must be mentioned that the octahedral and tetrahedral formulas of Theorems 3.5 and 4.3 facilitate the calculation of the coefficients in Love-Hunter expansions of the form (1.2), with $\left(v_{0}+\frac{1}{2}, \mu\right)$ equal to $\left( \pm \frac{1}{3}, \pm \frac{1}{4}\right)$ and $\left( \pm \frac{1}{4}, \pm \frac{1}{3}\right)$, respectively. Because these values satisfy $\mu \in\left(-\frac{1}{2}, \frac{1}{2}\right)$, the convergence result of [32] applies. 


\section{Ladder Operators, Lie Algebras, and Representations}

In the preceding sections, explicit formulas for the Legendre and Ferrers functions in the octahedral, tetrahedral, dihedral, and cyclic families were derived. Each such family (in the first-kind Ferrers case) is of the form $\left\{\mathrm{P}_{\nu_{0}+n}^{\mu_{0}+m}(z=\cos \theta)\right\}$, where $v_{0}, \mu_{0}$ are or may be fractional, and $(n, m)$ ranges over $\mathbb{Z}^{2}$. In this section, the connection between such a family and conventional $S O(3)$-based harmonic analysis on the sphere $S^{2}=S O(3) / S O(2)$, coordinatized by the angles $(\theta, \varphi)$, is briefly explored.

The connection goes through the corresponding family of generalized spherical harmonics, $\mathrm{P}_{v}^{\mu}(\cos \theta) \mathrm{e}^{\mathrm{i} \mu \varphi}$, with $(v, \mu) \in\left(v_{0}, \mu_{0}\right)+\mathbb{Z}^{2}$. But the connection is not as strong as one would like. If $\nu_{0}, \mu_{0}$ are rational but not integral, these harmonic functions will not be single-valued on the symmetric space $S^{2}$. (In the cases of interest here, each $\mathrm{P}_{v}^{\mu}(z)$ in the family is algebraic in $z$, and they can be viewed as finitevalued.) They may not be square-integrable, because the leading behavior of $\mathrm{P}_{v}^{\mu}(z)$ as $z \rightarrow 1^{-}$is proportional to $(1-z)^{-\mu / 2}$ unless $\mu$ is a positive integer.

For these reasons, the focus is on the action of Lie algebras (of 'infinitesimal transformations') on a function family of this type, specified by $\left(v_{0}, \mu_{0}\right)$, rather than the action of a Lie group such as $S O(3)$. The space spanned by the classical spherical harmonics $Y_{n}^{m}(\theta, \varphi) \propto \mathrm{P}_{n}^{m}(\cos \theta) \mathrm{e}^{\mathrm{i} m \varphi}$, with $n \geq 0$ and $m \in \mathbb{Z}$, admits an action of the rotation group $S O(3)$. The Lie algebra $\mathfrak{s o}(3, \mathbb{R})$ of $3 \times 3$ real skew-symmetric matrices can be represented by differential operators on $S^{2}$, with real coefficients, and acts on the space of spherical harmonics. The resulting infinite-dimensional representation is reducible: for $n=0,1,2, \ldots$, it includes the usual $(2 n+1)$-dimensional representation on the span of $Y_{n}^{-n}, \ldots, Y_{n}^{n}$. But $\mathfrak{s o}(3, \mathbb{R})$ is not the only Lie algebra to be considered.

A larger Lie algebra than $\mathfrak{s o}(3, \mathbb{R})$ acts naturally on the spherical harmonics, or rather, on the (regular) solid harmonics $r^{n} Y_{n}^{m}(\theta, \varphi)$, which satisfy Laplace's equation on $\mathbb{R}^{3}$. (See [28, Sect.3.6].) This is the 10-dimensional real Lie algebra $\mathfrak{s o}(4,1)$ that is generated by 'ladder' operators that increment and decrement the degree $n$, as well as the order $m$. They are represented by differential operators on $\mathbb{R}^{3}$, with real coefficients. The real span of these operators exponentiates to the Lie group $\mathrm{SO}_{0}(4,1)$, which contains as subgroups (i) the 3-parameter group $S O(3)$ of rotations about the origin, (ii) a 3-parameter Abelian group of translations of $\mathbb{R}^{3}$, (iii) a 1-parameter group of dilatations (linear scalings of $\mathbb{R}^{3}$ ), and (iv) a 3-parameter Abelian group of 'special conformal transformations.' The last are quadratic rational self-maps of $\mathbb{R}^{3}$ (or rather the real projective space $\mathbb{R P}^{3}$, because they can interchange finite and infinite points).

The preceding results, now standard, are extended below to any family of generalized solid harmonics $\left\{r^{\nu} \mathrm{P}_{\nu}^{\mu}(\cos \theta) \mathrm{e}^{\mathrm{i} \mu \varphi}\right\}$, with $(\nu, \mu) \in\left(\nu_{0}, \mu_{0}\right)+\mathbb{Z}^{2}$ for specified $v_{0}, \mu_{0}$. In Sect.9.1, the differential and nondifferential recurrences on $v$ and $\mu$ are derived. (See Theorems 9.1 and 9.2.) In Sect. 9.2, it is shown that the ladder operators in the differential recurrences generate a 10-dimensional real Lie algebra, and an isomorphism from this algebra not to $\mathfrak{s o}(4,1)$ but to $\mathfrak{s o}(3,2)$ is exhibited. The treatment closely follows Celeghini and del Olmo [4], but the explicit isomorphism in Theorem 9.3 is new.

In the setting of special function identities, which typically involve real linear combinations of differential operators, $\mathfrak{s o}(3,2)$ arises more naturally than does $\mathfrak{s o}(4,1)$. 
But by a limited form of complexification, $\mathfrak{s o}(3,2)$ can be converted to $\mathfrak{s o}(4,1)$, and indeed to $\mathfrak{s o}(5, \mathbb{R})$. These are alternative real forms of the rank- 2 complex Lie algebra $\mathfrak{s o}(5, \mathbb{C})$, to which they complexify, and the eight displacement vectors $\Delta(\nu, \mu)=$ $(0, \pm 1),( \pm 1,0),( \pm 1, \pm 1)$ can be identified with the roots of $\mathfrak{s o}(5, \mathbb{C})$.

In Sect. 9.3, it is shown that irrespective of $\left(v_{0}, \mu_{0}\right)$, the representation of $\mathfrak{s o}(3,2)$ [or of $\mathfrak{s o}(4,1)$ or $\mathfrak{s o}(5, \mathbb{R})]$ carried by the solid harmonics $r^{\nu} \mathrm{P}_{\nu}^{\mu}(\cos \theta) \mathrm{e}^{\mathrm{i} \mu \varphi}$ with $(\nu, \mu) \in\left(\nu_{0}, \mu_{0}\right)+\mathbb{Z}^{2}$ is of a special type: its quadratic Casimir operator takes a fixed value, and its quartic one vanishes. (See Theorem 9.6.) The former fact was found in [4], but the latter is new. The representation of $\mathfrak{s o}(3,2)$ on the solid harmonics of integer degree and order, and its representation on the ones of half-odd-integer degree and order, have irreducible constituents that are identified as the known Dirac singleton representations of $\mathfrak{s o}(3,2)$.

\subsection{Differential and Nondifferential Recurrences}

In any family $\left\{\mathrm{P}_{\nu_{0}+n}^{\mu_{0}+m}(z)\right\}_{(n, m) \in \mathbb{Z}^{2}}$, where $\mathrm{P}$ can be taken as any of $\mathrm{P}, \mathrm{Q}, P, \hat{Q}$, any three distinct members are linearly dependent, over the field of functions that are rational in $z$ and $\sqrt{1-z^{2}}$ (Ferrers case) or $\sqrt{z^{2}-1}$ (Legendre case). In particular, any three contiguous members are so related, by a three-term ladder recurrence.

The underlying recurrences are differential ones, which generally permit any single $\mathrm{P}_{v}^{\mu}$ and its derivative to generate any member contiguous to it, as a linear combination, and by iteration, to generate any $\mathrm{P}_{\nu+\Delta v}^{\mu+\Delta \mu}$ in which $\Delta(\nu, \mu) \in \mathbb{Z}^{2}$.

Theorem 9.1 The Ferrers functions $\mathrm{P}_{v}^{\mu}=\mathrm{P}_{v}^{\mu}(z)$ satisfy eight differential recurrences, divided into four ladders, i.e., \pm -pairs, with $\Delta(v, \mu)= \pm(0,1), \pm(1,0), \pm(1,1)$, and $\pm(1,-1)$. Each pair is of the form

$$
\alpha_{ \pm} \mathrm{P}_{v+\Delta v}^{\mu+\Delta \mu}=\mp z^{-\sigma_{0}^{ \pm}+\varepsilon_{0}}\left(1-z^{2}\right)^{-\sigma_{1}^{ \pm} / 2+\varepsilon_{1} / 2} \frac{\mathrm{d}}{\mathrm{d} z}\left[z^{\sigma_{0}^{ \pm}}\left(1-z^{2}\right)^{\sigma_{1}^{ \pm} / 2} \mathrm{P}_{v}^{\mu}\right]
$$

and for each pair, the exponents $\sigma_{0}^{ \pm}, \sigma_{1}^{ \pm}$, the exponents $\varepsilon_{0}, \varepsilon_{1}$, and the prefactor $\alpha_{ \pm}$ are given in Table 2. The second-kind functions $\mathrm{Q}_{\nu}^{\mu}$ satisfy identical recurrences.

The Legendre functions $P_{v}^{\mu}, Q_{v}^{\mu}$ [the latter unnormalized, i.e., the functions $\left.\mathrm{e}^{\mu \pi \mathrm{i}} \hat{Q}_{v}^{\mu}\right]$ satisfy recurrences obtained from the preceding by (i) multiplying the righthand side by a sign factor, equal to $\mathrm{i}^{\varepsilon_{1}+\Delta \mu}$, and (ii) replacing $1-z^{2}$ by $z^{2}-1$.

Proof The four nondiagonal recurrences on the order and degree, with $\Delta(v, \mu)=$ $\pm(0,1)$ and $\pm(1,0)$, are classical and can be found in many reference works $[11,31,36]$. They can be deduced from the differential recurrences of Jacobi, which increment or decrement the parameters of the function ${ }_{2} F_{1}(a, b ; c ; x)$. (See [11, 2.8(20)-2.8(27)].)

The final four diagonal ones, at least for $\mathrm{P}_{v}^{\mu}$ when $v, \mu$ are integers, are due to Celeghini and del Olmo [4]. Each can be derived from the nondiagonal ones by a tedious process of elimination, but the process can be systematized as the calculation of the commutator of two differential operators. (See Sect.9.2, below.) 
Table 2 Parameters for the differential recurrences of Theorem 9.1

\begin{tabular}{llll}
\hline$\Delta(\nu, \mu)$ & $\sigma_{0}^{ \pm}, \sigma_{1}^{ \pm}$ & $\varepsilon_{0}, \varepsilon_{1}$ & $\alpha_{ \pm}$ \\
\hline$\pm(0,1)$ & $0, \mp \mu$ & 0,1 & {$[1,(v+\mu)(v-\mu+1)]_{ \pm}$} \\
$\pm(1,0)$ & $0, \frac{1}{2} \pm\left(v+\frac{1}{2}\right)$ & 0,2 & {$[v-\mu+1, v+\mu]_{ \pm}$} \\
$\pm(1,1)$ & $\frac{1}{2} \pm\left(v+\frac{1}{2}\right) \pm \mu, \mp \mu$ & 1,1 & {$[1,(v+\mu)(v+\mu-1)]_{ \pm}$} \\
$\pm(1,-1)$ & $-\frac{1}{2} \pm\left(v+\frac{1}{2}\right) \mp \mu, \pm \mu$ & 1,1 & {$[(v-\mu+1)(v-\mu+2), 1]_{ \pm}$}
\end{tabular}

In the rightmost column, the notation $[a \mid b]_{ \pm}$signifies $a$, resp. $b$, in the + , resp. - case

The differential recurrences satisfied by $\mathrm{P}_{v}^{\mu}$ can be written in circular-trigonometric forms that will be needed below. Substituting $z=\cos \theta$ yields

$$
\begin{aligned}
& \alpha_{ \pm} \mathrm{P}_{v}^{\mu \pm 1}=\left[ \pm D_{\theta}-\mu \cot \theta\right] \mathrm{P}_{v}^{\mu}, \\
& \alpha_{ \pm} \mathrm{P}_{v \pm 1}^{\mu}=\left\{ \pm(\sin \theta) D_{\theta}+\left[\left(v+\frac{1}{2}\right) \pm \frac{1}{2}\right] \cos \theta\right\} \mathrm{P}_{v}^{\mu}, \\
& \alpha_{ \pm} \mathrm{P}_{v \pm 1}^{\mu \pm 1}=\left\{ \pm(\cos \theta) D_{\theta}-\mu \csc \theta+\left[-\left(v+\frac{1}{2}\right) \mp \frac{1}{2}\right] \sin \theta\right\} \mathrm{P}_{v}^{\mu}, \\
& \alpha_{ \pm} \mathrm{P}_{v \pm 1}^{\mu \mp 1}=\left\{\mp(\cos \theta) D_{\theta}-\mu \csc \theta+\left[+\left(v+\frac{1}{2}\right) \pm \frac{1}{2}\right] \sin \theta\right\} \mathrm{P}_{v}^{\mu},
\end{aligned}
$$

which are satisfied by $\mathrm{P}_{v}^{\mu}=\mathrm{P}_{v}^{\mu}(\cos \theta)$. Here, $D_{\theta}:=\mathrm{d} / \mathrm{d} \theta$, and the four prefactors $\alpha_{ \pm}$ are listed in the last column of Table 2, in order. The recurrences (9.1c) and (9.1d) have appeared in the literature but are not well known; the only appearances that we have found, with $v, \mu$ restricted to integer values, are in [1] and [17, Sect. A.2]. Equations (9.1c) and (9.1d) imply each other because $\mathrm{P}_{-v-1}^{\mu}=\mathrm{P}_{v}^{\mu}$ for all $v, \mu$. That is, $\mathrm{P}_{v}^{\mu}$ is unaffected by the negating of the shifted degree parameter $v+\frac{1}{2}$.

The three-term ladder recurrences derived from the four pairs of differential recurrences are given in the following theorem. The diagonal ones, coming from the ladders with $\Delta(\nu, \mu)= \pm(1,1)$ and $\pm(1,-1)$, appear to be new.

Theorem 9.2 The Ferrers functions $\mathrm{P}_{v}^{\mu}=\mathrm{P}_{v}^{\mu}(z)$ satisfy second-order (i.e., threeterm) recurrences on the order $\mu$ and degree $v$, namely

$$
\begin{aligned}
& \sqrt{1-z^{2}} \mathrm{P}_{v}^{\mu+1}+2 \mu z \mathrm{P}_{v}^{\mu}+(v+\mu)(v-\mu+1) \sqrt{1-z^{2}} \mathrm{P}_{v}^{\mu-1}=0 \\
& (v-\mu+1) \mathrm{P}_{v+1}^{\mu}-(2 v+1) z \mathrm{P}_{v}^{\mu}+(v+\mu) \mathrm{P}_{v-1}^{\mu}=0
\end{aligned}
$$

and the two diagonal recurrences

$$
\begin{aligned}
& \sqrt{1-z^{2}} \mathrm{P}_{v \pm 1}^{\mu+1}(z)+\left[ \pm(2 v+1)\left(1-z^{2}\right)+2 \mu\right] \mathrm{P}_{v}^{\mu}(z) \\
& +\left[\left(v+\frac{1}{2}\right) \pm\left(\mu-\frac{1}{2}\right)\right]\left[\left(v+\frac{1}{2}\right) \pm\left(\mu-\frac{3}{2}\right)\right] \sqrt{1-z^{2}} \mathrm{P}_{v \mp 1}^{\mu-1}(z)=0 .
\end{aligned}
$$

The second-kind functions $\mathrm{Q}_{v}^{\mu}$ satisfy identical second-order recurrences.

The Legendre functions $P_{v}^{\mu}, Q_{v}^{\mu}$ (the latter unnormalized, as above), satisfy recurrences obtained from the preceding by (i) multiplying each term containing a function 
of order $\mu+\delta$ and a coefficient proportional to $\left[\sqrt{1-z^{2}}\right]^{\alpha}$ by a sign factor, equal to $\mathrm{i}^{\alpha-\delta}$, and (ii) replacing $\sqrt{1-z^{2}}$ by $\sqrt{z^{2}-1}$.

Proof Eliminate the derivative terms from the recurrences of Theorem 9.1. This is the procedure used to derive Gauss's three-term, nearest-neighbor 'contiguous function relations' for ${ }_{2} F_{1}(a, b ; c ; x)$ from Jacobi's differential recurrences on $a, b ; c$.

It was noted in Sect. 2 that if $v+\mu$ is a negative integer, $Q_{v}^{\mu}$ and $Q_{v}^{\mu}$ are generally undefined (though there are exceptions). The recurrences for $\mathrm{Q}_{\nu}^{\mu}$ and $Q_{\nu}^{\mu}$ in Theorems 9.1 and 9.2 remain valid in a limiting sense even when $(\nu, \mu)$ is such that one or more of the functions involved is undefined.

\subsection{Lie Algebras}

The raising and lowering of the degree and order, in any doubly indexed family of (generalized) solid harmonics

$$
\mathcal{S}_{v}^{\mu}=\mathcal{S}_{v}^{\mu}(r, \theta, \varphi):=r^{\nu} \mathrm{P}_{v}^{\mu}(\cos \theta) \mathrm{e}^{\mathrm{i} \mu \varphi},
$$

where $(\nu, \mu) \in\left(v_{0}, \mu_{0}\right)+\mathbb{Z}^{2}$, can be performed by differential operators that do not need to depend explicitly on $\left(v_{0}, \mu_{0}\right)$ if they are allowed to involve, instead, the derivative operators $D_{r}, D_{\varphi}$ in addition to $D_{\theta}$.

The basic idea is due to Miller [26], and there is freedom in its implementation: either or both of the factors $r^{\nu}=\mathrm{e}^{\nu \log r}$ and $\mathrm{e}^{\mathrm{i} \mu \varphi}$ could include an ' $\mathrm{i}$ ' in its exponent, and the Ferrers functions $\mathrm{P}_{v}^{\mu}(\cos \theta)$ could be replaced by the Legendre ones $P_{v}^{\mu}(\cosh \xi)$. With the choices made in (9.2), $\mathcal{S}_{v}^{\mu}$ can be viewed as a (typically multi-valued) function of the spherical coordinates $r, \theta, \varphi$, which satisfies Laplace's equation on $\mathbb{R}^{3}$. Define ladder operators by

$$
\begin{aligned}
J_{ \pm} & =\mathrm{e}^{ \pm \mathrm{i} \varphi}\left[ \pm D_{\theta}+\mathrm{i}(\cot \theta) D_{\varphi}\right], \\
K_{ \pm} & =r^{ \pm 1}\left[ \pm(\sin \theta) D_{\theta}+(\cos \theta)\left(r D_{r}+\frac{1}{2} \pm \frac{1}{2}\right)\right], \\
R_{ \pm} & =r^{ \pm 1} \mathrm{e}^{ \pm \mathrm{i} \varphi}\left[ \pm(\cos \theta) D_{\theta}+\mathrm{i}(\csc \theta) D_{\varphi}-(\sin \theta)\left(r D_{r}+\frac{1}{2} \pm \frac{1}{2}\right)\right], \\
S_{ \pm} & =r^{ \pm 1} \mathrm{e}^{\mp \mathrm{i} \varphi}\left[\mp(\cos \theta) D_{\theta}+\mathrm{i}(\csc \theta) D_{\varphi}+(\sin \theta)\left(r D_{r}+\frac{1}{2} \pm \frac{1}{2}\right)\right] .
\end{aligned}
$$

Then, the differential recurrences (9.1) can be rewritten in terms of the $\mathcal{S}_{v}^{\mu}$ as

$$
\begin{aligned}
J_{ \pm} \mathcal{S}_{v}^{\mu} & =[1,(v+\mu)(v-\mu+1)]_{ \pm} \mathcal{S}_{v}^{\mu \pm 1}, \\
K_{ \pm} \mathcal{S}_{v}^{\mu} & =[v-\mu+1, v+\mu]_{ \pm} \mathcal{S}_{v \pm 1}^{\mu}, \\
R_{ \pm} \mathcal{S}_{v}^{\mu} & =[1,(v+\mu)(v+\mu-1)]_{ \pm} \mathcal{S}_{v \pm 1}^{\mu \pm 1}, \\
S_{ \pm} \mathcal{S}_{v}^{\mu} & =[(v-\mu+1)(v-\mu+2), 1]_{ \pm} \mathcal{S}_{v \pm 1}^{\mu \mp 1} .
\end{aligned}
$$

For each $\left(v_{0}, \mu_{0}\right)$, the solid harmonics $\left\{\mathcal{S}_{v}^{\mu}=\mathcal{S}_{\nu_{0}+n}^{\mu_{0}+m}\right\}$, or more accurately their real linear span, carry a representation of $J_{ \pm}, K_{ \pm}, R_{ \pm}, S_{ \pm}$. Generating a Lie algebra by working commutators out, one finds (with $[A, B]$ signifying $A B-B A$ ) 


$$
R_{ \pm}= \pm\left[J_{ \pm}, K_{ \pm}\right], \quad S_{ \pm}= \pm\left[J_{\mp}, K_{ \pm}\right]
$$

which explains why the diagonal recurrences in Theorems 9.1 and 9.2 can be most efficiently obtained by commutator calculations, as claimed.

It is useful additionally to define 'labeling' or 'maintaining' operators $J_{3}, K_{3}$ by

$$
J_{3}=-\mathrm{i} D_{\varphi}, \quad K_{3}=r D_{r}+\frac{1}{2},
$$

so that

$$
J_{3} \mathcal{S}_{v}^{\mu}=\mu \mathcal{S}_{v}^{\mu}, \quad K_{3} \mathcal{S}_{v}^{\mu}=\left(v+\frac{1}{2}\right) \mathcal{S}_{v}^{\mu} .
$$

By further calculations, one finds that the real Lie algebra generated by $J_{ \pm}, K_{ \pm}$closes, in the sense that it is finite-dimensional. In particular,

$$
\begin{aligned}
{\left[J_{3}, J_{ \pm}\right] } & = \pm J_{ \pm}, & {\left[J_{+}, J_{-}\right] } & =2 J_{3}, \\
{\left[K_{3}, K_{ \pm}\right] } & = \pm K_{ \pm}, & {\left[K_{+}, K_{-}\right] } & =-2 K_{3}, \\
{\left[R_{3}, R_{ \pm}\right] } & = \pm 2 R_{ \pm}, & {\left[R_{+}, R_{-}\right] } & =-4 R_{3}, \\
{\left[S_{3}, S_{ \pm}\right] } & = \pm 2 S_{ \pm}, & {\left[S_{+}, S_{-}\right] } & =-4 S_{3},
\end{aligned}
$$

where $R_{3}:=K_{3}+J_{3}$ and $S_{3}:=K_{3}-J_{3}$. To interpret these, recall that any real linear space with basis $\left\{X_{+}, X_{-}, X_{3}\right\}$, given a Lie algebra structure by

$$
\left[X_{3}, X_{ \pm}\right]= \pm X_{ \pm}, \quad\left[X_{+}, X_{-}\right]=2 \sigma X_{3}
$$

is isomorphic to $\mathfrak{s o}(3, \mathbb{R})$ if $\sigma>0$, and to $\mathfrak{s o}(2,1)$ (or equivalently $\mathfrak{s l}(2, \mathbb{R})$ ) if $\sigma<0$. Hence, the real Lie algebras spanned by $\left\{J_{+}, J_{-}, J_{3}\right\},\left\{K_{+}, K_{-}, K_{3}\right\},\left\{R_{+}, R_{-}, R_{3}\right\}$, and $\left\{S_{+}, S_{-}, S_{3}\right\}$, coming from the ladders with $\Delta(\nu, \mu)= \pm(0,1), \pm(1,0), \pm(1,1)$, and $\pm(1,-1)$, are isomorphic to $\mathfrak{s o}(3, \mathbb{R})$ (the first) and $\mathfrak{s o}(2,1)$ (the remaining three). The last two turn out to commute.

The real Lie algebra generated by $J_{ \pm}, K_{ \pm}$, of which these copies of $\mathfrak{s o}(3, \mathbb{R})$ and $\mathfrak{s o}(2,1)$ are subalgebras, is 10 -dimensional and is spanned over $\mathbb{R}$ by $J_{ \pm}, J_{3} ; K_{ \pm}, K_{3}$; $R_{ \pm} ; S_{ \pm}$. It of course has real structure constants. For any $\left(v_{0}, \mu_{0}\right)$, its representation by differential operators on $\mathbb{R}^{3}$, as above, is carried by the real span of the solid harmonics $\mathcal{S}_{v_{0}+n}^{\mu_{0}+m},(n, m) \in \mathbb{Z}^{2}$. This result was obtained by Celeghini and del Olmo [4], though they confined themselves to integer $v, \mu$, i.e., in effect to $\left(v_{0}, \mu_{0}\right)=(0,0){ }^{1}$

To identify this 10-dimensional real algebra, it is useful to relabel its basis elements. First, let

$$
\left(P_{+}, P_{-}, P_{3}\right):=\left(S_{+},-R_{-}, K_{-}\right), \quad\left(C_{+}, C_{-}, C_{3}\right):=\left(-R_{+}, S_{-}, K_{+}\right)
$$

in each of which the three elements commute. The algebra can then be viewed as the span over $\mathbb{R}$ of $J_{ \pm}, J_{3} ; P_{ \pm}, P_{3} ; C_{ \pm}, C_{3}$ and $K_{3}$, which will be written as $D$ henceforth.

\footnotetext{
1 The reader of [4] should note that in Sect. 5, $R_{ \pm}:=\left[K_{ \pm}, J_{ \pm}\right]$and $S_{ \pm}:=\left[K_{ \pm}, J_{\mp}\right]$ should be emended to read $R_{ \pm}:=\mp\left[K_{ \pm}, J_{ \pm}\right]$and $S_{ \pm}:=\mp\left[K_{ \pm}, J_{\mp}\right]$.
} 
Define

$$
\begin{aligned}
\left(P C_{+}^{ \pm}, P C_{-}^{ \pm}, P C_{3}^{ \pm}\right) & :=\frac{1}{2}\left[\left(P_{+}, P_{-}, P_{3}\right) \pm\left(C_{+}, C_{-}, C_{3}\right)\right] \\
& =\frac{1}{2}\left(\mp R_{+}+S_{+},-R_{-} \pm S_{-}, K_{-} \pm K_{+}\right) .
\end{aligned}
$$

Also, for $X=J, P, C, P C^{+}, P C^{-}$, define the 'skew-Cartesian' elements

$$
x_{1}:=\left(X_{+}+X_{-}\right) / 2, \quad x_{2}:=\left(X_{+}-X_{-}\right) / 2, \quad x_{3}:=X_{3},
$$

so that $X_{ \pm}=X_{1} \pm X_{2}$. The algebra will then be the real span of $\mathcal{J}_{1}, \mathcal{J}_{2}, \mathcal{J}_{3}, \mathcal{P}_{1}, \mathcal{P}_{2}, \mathcal{P}_{3}$; $\mathcal{C}_{1}, \mathcal{C}_{2}, \mathcal{C}_{3} ; D$, or equivalently of $\mathcal{J}_{1}, \mathcal{\partial}_{2}, \mathcal{J}_{3} ; \mathcal{P C}_{1}^{ \pm}, \mathcal{P C}_{2}^{ \pm}, \mathcal{P} \mathcal{C}_{3}^{ \pm} ; D$.

It is readily verified that $\mathcal{J}_{i}$ commutes with $\mathcal{P} \mathcal{C}_{i}^{+}$and $\mathcal{P} \mathcal{C}_{i}^{-}$for $i=1,2,3$, and that

$$
\begin{aligned}
{\left[\mathcal{\partial}_{i}, \mathcal{J}_{j}\right] } & =\{-1,+1,-1\}_{k} \mathcal{J}_{k}, \\
{\left[\mathcal{J}_{i}, \mathcal{P} \mathcal{C}_{j}^{ \pm}\right] } & =\{-1,+1,-1\}_{k} \mathcal{P C}_{k}^{ \pm}, \\
{\left[\mathcal{P C}_{i}^{ \pm}, \mathcal{P} \mathcal{C}_{j}^{ \pm}\right] } & =\mp\{-1,+1,-1\}_{k} \mathcal{J}_{k},
\end{aligned}
$$

where $i, j, k$ is any cyclic permutation of $1,2,3$, with $\{a, b, c\}_{k}$ meaning $a, b, c$ when $k=1,2,3$. Also, the $3 \times 3$ matrix of commutators $\left[\mathcal{P C}_{i}^{+}, \mathcal{P C}_{j}^{-}\right.$] indexed by $1 \leq i, j \leq 3$ equals diag $(-D,+D,-D)$. Additionally,

$$
\left[D, \mathcal{J}_{i}\right]=0, \quad\left[D, \mathcal{P} \mathcal{C}_{i}^{ \pm}\right]=-\mathcal{P} \mathcal{C}_{i}^{\mp},
$$

for $i=1,2,3$. These identities specify the structure of the algebra.

Now, recall that the real Lie algebra $\mathfrak{s o}(p, q)$ with $p+q=n$ has the following defining representation. If $\Gamma=\left(g_{i j}\right)=\operatorname{diag}(+1, \ldots,+1,-1, \ldots,-1)$, with $q+1$ 's and $p-1$ 's, then $\mathfrak{s o}(p, q)$ comprises all real $n \times n$ matrices $A$ for which $\Gamma A$ is skewsymmetric. There is a sign convention here, and a $p \leftrightarrow q$ symmetry; without loss of generality, $p \geq q$ will be assumed. It is sometimes useful to permute the +1 's and -1 's.

More concretely, $\mathfrak{s o}(p, q)$ can be realized as the real span of the $n \times n$ matrices $\mathbf{M}_{a b}$, $1 \leq a<b \leq n$, where $\mathbf{M}_{a b}=\Gamma \mathcal{E}_{a b}-\mathcal{E}_{b a} \Gamma$. In this, $\mathcal{E}_{a b}$ is the $n \times n$ matrix with a 1 in row $a$, column $b$, and zeroes elsewhere. One often extends the size- $\left(\begin{array}{l}n \\ 2\end{array}\right)$ basis $\left\{\mathbf{M}_{a b}\right\}$ to a 'tensor operator,' i.e., a skew-symmetric $n \times n$ matrix of elements $\left(\mathbf{M}_{a b}\right)$, by requiring that $\mathbf{M}_{b a}=-\mathbf{M}_{a b}$ for $1 \leq a, b \leq n$. The commutation relations

$$
\left[\mathbf{M}_{a b}, \mathbf{M}_{c d}\right]=g_{a d} \mathbf{M}_{b c}+g_{b c} \mathbf{M}_{a d}-g_{a c} \mathbf{M}_{b d}-g_{b d} \mathbf{M}_{a c}
$$

are easily checked. 
Theorem 9.3 The real Lie algebra generated by $J_{ \pm}, K_{ \pm}$is isomorphic to $\mathfrak{s o}(3,2)$, an isomorphism being specified by the tensor operator

$$
\left(\mathbf{M}_{a b}\right)=\left(\begin{array}{cc|ccc}
0 & \mathcal{P C}_{2}^{-} & -\mathcal{P C}_{1}^{-} & -\mathcal{P C}_{3}^{-} & -D \\
-\mathcal{P} \mathcal{C}_{2}^{-} & 0 & \partial_{3} & -\mathcal{J}_{1} & \mathcal{P} \mathcal{C}_{2}^{+} \\
\hline \mathcal{P C}_{1}^{-} & -\mathcal{J}_{3} & 0 & \mathcal{J}_{2} & -\mathcal{P C}_{1}^{+} \\
\mathcal{P C}_{3}^{-} & \mathcal{J}_{1} & -\mathcal{J}_{2} & 0 & -\mathcal{P C}_{3}^{+} \\
D & -\mathcal{P C}_{2}^{+} & \mathcal{P C}_{1}^{+} & \mathcal{P C}_{3}^{+} & 0
\end{array}\right)
$$

with $\Gamma=\operatorname{diag}(+1,+1,-1,-1,-1)$.

Proof The proof is by inspection.

This $\mathfrak{s o}(3,2)$ has the Cartan decomposition $\mathfrak{k} \oplus_{\mathbb{R}} \mathfrak{p}$, where

$$
\begin{aligned}
& \mathfrak{k}=\operatorname{sp}_{\mathbb{R}}\left\{\mathcal{J}_{2}, \mathcal{P} \mathcal{C}_{1}^{+}, \mathcal{P} \mathcal{C}_{3}^{+}, \mathcal{P} \mathcal{C}_{2}^{-}\right\} \\
& \mathfrak{p}=\operatorname{sp}_{\mathbb{R}}\left\{\mathcal{J}_{1}, \mathcal{J}_{3}, \mathcal{P} \mathcal{C}_{2}^{+}, \mathcal{P} \mathcal{C}_{1}^{-}, \mathcal{P} \mathcal{C}_{3}^{-}, D\right\}
\end{aligned}
$$

are the 'compact' and 'noncompact' subspaces. (The terms refer to the Lie subgroups of $S_{0}(3,2)$ to which they exponentiate.) Real Lie algebras isomorphic to $\mathfrak{s o}(4,1)$ and $\mathfrak{s o}(5, \mathbb{R})$ can be obtained by Weyl's trick of redefining some or all of the basis elements of $\mathfrak{p}$ to include ' $\mathrm{i}$ ' factors. In doing this, a slightly changed notation will be useful. For $X=J, P, C, P C^{+}, P C^{-}$, define the 'Cartesian' elements

$$
X_{1}:=\left(X_{+}+X_{-}\right) / 2, \quad X_{2}:=-\mathrm{i}\left(X_{+}-X_{-}\right) / 2,
$$

so that $X_{ \pm}=X_{1} \pm \mathrm{i} X_{2}$ and $\left(X_{1}, X_{2}, X_{3}\right)=\left(X_{1}, \mathrm{i} X_{2}, X_{3}\right)$.

Theorem 9.4 (i) The real span of $\mathrm{i} J_{1}, \mathrm{i} J_{2}, \mathrm{i} J_{3} ; P_{1}, P_{2}, P_{3} ; C_{1}, C_{2}, C_{3} ; D$, or equivalently of $\mathrm{i} J_{1}, \mathrm{i} J_{2}, \mathrm{i} J_{3} ; P C_{1}^{ \pm}, P C_{2}^{ \pm}, P C_{3}^{ \pm} ; D$, is a real Lie algebra isomorphic to $\mathfrak{s o}(4,1)$, an isomorphism being specified by the tensor operator

$$
\left(\mathbf{M}_{a b}\right)=\left(\begin{array}{c|cccc}
0 & -P C_{1}^{-} & -P C_{2}^{-} & -P C_{3}^{-} & -D \\
\hline P C_{1}^{-} & 0 & -\mathrm{i} J_{3} & \mathrm{i} J_{2} & -P C_{1}^{+} \\
P C_{2}^{-} & \mathrm{i} J_{3} & 0 & -\mathrm{i} J_{1} & -P C_{2}^{+} \\
P C_{3}^{-} & -\mathrm{i} J_{2} & \mathrm{i} J_{1} & 0 & -P C_{3}^{+} \\
D & P C_{1}^{+} & P C_{2}^{+} & P C_{3}^{+} & 0
\end{array}\right),
$$

with $\Gamma=\operatorname{diag}(+1,-1,-1,-1,-1)$.

(ii) The real span of $\mathrm{i} J_{1}, \mathrm{i} J_{2}, \mathrm{i} J_{3} ; \mathrm{i} P C_{1}^{+}, \mathrm{i} P C_{2}^{+}, \mathrm{i} P C_{3}^{+} ; P C_{1}^{-}, P C_{2}^{-}, P C_{3}^{-} ; \mathrm{i} D$ is a real Lie algebra isomorphic to $\mathfrak{s o}(3,2)$, an isomorphism being specified by the tensor operator

$$
\left(\mathbf{M}_{a b}\right)=\left(\begin{array}{c|ccc|c}
0 & -P C_{1}^{-} & -P C_{2}^{-} & -P C_{3}^{-} & -\mathrm{i} D \\
\hline P C_{1}^{-} & 0 & -\mathrm{i} J_{3} & \mathrm{i} J_{2} & -\mathrm{i} P C_{1}^{+} \\
P C_{2}^{-} & \mathrm{i} J_{3} & 0 & -\mathrm{i} J_{1} & -\mathrm{i} P C_{2}^{+} \\
P C_{3}^{-} & -\mathrm{i} J_{2} & \mathrm{i} J_{1} & 0 & -\mathrm{i} P C_{3}^{+} \\
\hline \mathrm{i} D & \mathrm{i} P C_{1}^{+} & \mathrm{i} P C_{2}^{+} & \mathrm{i} P C_{3}^{+} & 0
\end{array}\right),
$$


with $\Gamma=\operatorname{diag}(+1,-1,-1,-1,+1)$.

Proof (i) Multiply the second row and the second column of the $\left(\mathbf{M}_{a b}\right)$ in Theorem 9.3 by 'i', and (innocuously) interchange the second and third rows, and the second and third columns. (ii) Continuing (or in a sense reversing), multiply the last row and the last column by 'i'.

Theorem 9.5 The real span of $\mathrm{i} J_{1}, \mathrm{i} J_{2}, \mathrm{i} J_{3} ; P C_{1}^{+}, P C_{2}^{+}, P C_{3}^{+}$; i $P C_{1}^{-}, \mathrm{i} P C_{2}^{-}$, i $P C_{3}^{-} ;$i $D$ is a real Lie algebra isomorphic to $\mathfrak{s o}(5, \mathbb{R})$, an isomorphism being specified by the tensor operator

$$
\left(\mathbf{M}_{a b}\right)=\left(\begin{array}{ccccc}
0 & -\mathrm{i} P C_{1}^{-} & -\mathrm{i} P C_{2}^{-} & -\mathrm{i} P C_{3}^{-} & -\mathrm{i} D \\
\mathrm{i} P C_{1}^{-} & 0 & -\mathrm{i} J_{3} & \mathrm{i} J_{2} & -P C_{1}^{+} \\
\mathrm{i} P C_{2}^{-} & \mathrm{i} J_{3} & 0 & -\mathrm{i} J_{1} & -P C_{2}^{+} \\
\mathrm{i} P C_{3}^{-} & -\mathrm{i} J_{2} & \mathrm{i} J_{1} & 0 & -P C_{3}^{+} \\
\mathrm{i} D & P C_{1}^{+} & P C_{2}^{+} & P C_{3}^{+} & 0
\end{array}\right),
$$

with $\Gamma=\operatorname{diag}(-1,-1,-1,-1,-1)$.

Proof Multiply the first row and the first column of the $\left(\mathbf{M}_{a b}\right)$ in part (i) of Theorem 9.4 by 'i'.

With ' $i$ ' factors in basis elements, the $\mathfrak{s o}(3,2), \mathfrak{s o}(4,1)$ and $\mathfrak{s o}(5, \mathbb{R})$ of Theorems 9.4 and 9.5 look awkward. But it follows from (9.3), (9.5), and (9.12) that each basis element in the $\mathfrak{s o}(4,1)$ of Theorem 9.4(i), i.e., each of $\mathrm{i} J_{i}, P_{i}, C_{i}$, and $D$, is realized by a differential operator in $r, \theta, \varphi$ with real coefficients. This is not the case for the basis elements of $\mathfrak{s o}(3,2)$ and $\mathfrak{s o}(5, \mathbb{R})$.

The geometric significance of this realization of $\mathfrak{s o}(4,1)$ is revealed by changing from spherical coordinates $(r, \theta, \varphi)$ to Cartesian ones, $\left(x_{1}, x_{2}, x_{3}\right)$ on $\mathbb{R}^{3}$. Using

$$
x:=\left(x_{1}, x_{2}, x_{3}\right)=(r \sin \theta \cos \varphi, r \sin \theta \sin \varphi, r \cos \theta),
$$

one finds that

$$
\begin{array}{rlrl}
\mathrm{i} J_{i} & =x_{j} \partial_{k}-x_{k} \partial_{j}, & & i=1,2,3, \\
P_{i} & =\partial_{i}, & i & i=1,2,3, \\
C_{i} & =x_{i}-(x \cdot x) \partial_{i}+2 x_{i}(x \cdot \partial), & i & =1,2,3, \\
D & =x \cdot \partial+\frac{1}{2}, & &
\end{array}
$$

where $\partial_{i}:=\mathrm{d} / \mathrm{d} x_{i}$ and $i, j, k$ is a cyclic permutation of $1,2,3$. That is, the $\mathrm{i} J_{i}$ generate rotations about the origin, the $P_{i}$ generate translations, and $D$ generates dilatations (linear scalings of $\mathbb{R}^{3}$ ). The $C_{i}$ generate special conformal transformations, which are degree-2 rational maps of $\mathbb{R}^{3}$ (or rather $\mathbb{R P}^{3}$ ) to itself. The commutation relations

$$
\begin{aligned}
{\left[J_{i}, J_{j}\right] } & =\mathrm{i} \epsilon_{i j k} J_{k}, & {\left[P C_{i}^{ \pm}, P C_{j}^{ \pm}\right] } & =\mp \mathrm{i} \epsilon_{i j k} J_{k}, \\
{\left[J_{i}, P C_{j}^{ \pm}\right] } & =\mathrm{i} \epsilon_{i j k} P C_{k}^{ \pm}, & {\left[P C_{i}^{+}, P C_{j}^{-}\right] } & =-\delta_{i j} D, \\
{\left[D, J_{i}\right] } & =0, & {\left[D, P C_{i}^{ \pm}\right] } & =-P C_{i}^{\mp},
\end{aligned}
$$


written in terms of $P C_{i}^{ \pm}=\frac{1}{2}\left(P_{i} \pm C_{i}\right)$, follow either from (9.9), (9.10), from (9.11), or from (9.13). Here, the summation convention of tensor analysis is employed. The Levi-Cività tensor $\epsilon_{i j k}$ is skew-symmetric in all indices, with $\epsilon_{123}=+1$, and $\delta_{i j}$ is the Kronecker delta. Together with

$$
J_{1}=\frac{1}{2}\left(J_{+}+J_{-}\right), \quad J_{2}=-\frac{\mathrm{i}}{2}\left(J_{+}-J_{-}\right), \quad D=K_{3},
$$

the formulas

$$
\begin{aligned}
& P C_{1}^{ \pm}=\frac{1}{4}\left(\mp R_{+}-R_{-} \pm S_{+}+S_{-}\right), \\
& P C_{2}^{ \pm}=-\frac{\mathrm{i}}{4}\left(\mp R_{+}+R_{-} \mp S_{+}+S_{-}\right), \\
& P C_{3}^{ \pm}=\frac{1}{2}\left( \pm K_{+}+K_{-}\right),
\end{aligned}
$$

express all these differential operators in terms of the original $J_{ \pm}, K_{ \pm}, R_{ \pm}, S_{ \pm} ; J_{3}, K_{3}$ of (9.3), (9.5).

The ten operators in $(9.13)$ span (over $\mathbb{R}$ ) the Lie algebra of conformal differential operators on $\mathbb{R}^{3}$, which is known to have an $\mathfrak{s o}(4,1)$ structure. (See Miller [28, Sect. 3.6].) This is the symmetry algebra of the Laplacian $\nabla^{2}$ on $\mathbb{R}^{3}$, which comprises all real first-order operators $L$ for which $\left[L, \nabla^{2}\right] \propto \nabla^{2}$, i.e., for which $\left[L, \nabla^{2}\right]$ has $\nabla^{2}$ as a right factor. It can be viewed as acting on any suitable space of functions on $\mathbb{R}^{3}$, and exponentiates to the group $S_{0}(4,1)$ of conformal transformations, realized as flows on $\mathbb{R}^{3}$ (or $\mathbb{R P}^{3}$ ). But the starting point used here was their action on the span of the generalized solid harmonics $\mathcal{S}_{\nu_{0}+n}^{\mu_{0}+m}$ with $(n, m) \in \mathbb{Z}^{2}$, which are (multi-valued) solutions of Laplace's equation.

In the physics literature on conformal Lie algebras and groups, the terms ' $x_{i}$ ' in $(9.13 \mathrm{c})$ and ' $\frac{1}{2}$ ' in $(9.13 \mathrm{~d})$ often appear as $2 \delta x_{i}$ and $\delta$ respectively, where $\delta$ is the so-called scaling dimension; though the resulting commutation relations do not involve $\delta$. The value $\delta=\frac{1}{2}$ is specific to the symmetry algebra of the Laplacian.

There are many variations on the present technique of using differential recurrences to construct real Lie algebras, realized by differential operators, that are isomorphic to the real forms of $\mathfrak{s o}(5, \mathbb{C})$. The solid harmonics $\mathcal{S}_{v}^{\mu}$ that were employed here are extensions to $\mathbb{R}^{3}$ of the (surface) spherical harmonics $\mathrm{P}_{\nu}^{\mu}(\cos \theta) \mathrm{e}^{\mathrm{i} \mu \varphi}$ on the symmetric space $S^{2}=S O(3) / S O(2)$. If not Ferrers but Legendre functions were used, the starting point would be the hyperboloidal ones $P_{\nu}^{\mu}(\cosh \xi) \mathrm{e}^{\mathrm{i} \mu \varphi}$, defined using coordinates $(\xi, \varphi)$ on the hyperboloid $H^{2}=S O(2,1) / S O(2)$, i.e., the surface $x_{1}^{2}+x_{2}^{2}-x_{3}^{2}+$ const $=0$. Their extensions to $\mathbb{R}^{3}$ satisfy the $(2+1)$-dimensional wave equation, rather than Laplace's equation. (See [28, Chap. 4] and [9].) But isomorphic algebras could be constructed.

\subsection{Lie Algebra Representations}

In Sect. 9.2, it was shown that for any $\left(v_{0}, \mu_{0}\right)$, there are representations of the real Lie algebras $\mathfrak{s o}(3,2), \mathfrak{s o}(4,1), \mathfrak{s o}(5, \mathbb{R})$ that are carried by the span of the family of (generically multi-valued) solid harmonics $\mathcal{S}_{\nu}^{\mu}(r, \theta, \varphi),(v, \mu) \in\left(v_{0}, \mu_{0}\right)+\mathbb{Z}^{2}$. These 
arise from the action of the ladder operators on the Ferrers functions $\mathrm{P}_{v}^{\mu}(\cos \theta)$. Solid harmonics are harmonic functions on $\mathbb{R}^{3}$, satisfying Laplace's equation, and the ones in the octahedral, tetrahedral, dihedral, and cyclic families are or can be finite-valued.

These infinite-dimensional representations are restrictions of the representation of the common complexification $\mathfrak{s o}(5, \mathbb{C})$, which is carried by the (complex) span of the family. They are generically irreducible and are also generically non-skew-Hermitian, so that except in special cases, they do not exponentiate to unitary representations of the corresponding Lie groups, even formally. This will now be investigated.

Each of the three real Lie algebras is of rank 2, so the center of its universal enveloping algebra is generated by two elements, called Casimir invariants, and any irreducible representation must represent each Casimir by a constant. The analysis of such representations resembles the unified classification of the irreducible representations of $\mathfrak{s o}(2,1)$ and $\mathfrak{s o}(3, \mathbb{R})$, the real forms of $\mathfrak{s o}(3, \mathbb{C})$, which is well known. (See, e.g., [8, Chap. 3].) In this, representations are classified by the value taken by their (single) Casimir, and by their reductions with respect to a (1-dimensional) Cartan subalgebra. This leads to an understanding of which representations are skew-Hermitian and which are finite-dimensional. However, no comparable unified approach to all representations of $\mathfrak{s o}(3,2), \mathfrak{s o}(4,1)$, and $\mathfrak{s o}(5, \mathbb{R})$ seems to have been published. The literature has dealt almost exclusively with the skew-Hermitian ones. $(\mathfrak{s o}(3,2)$ and $\mathfrak{s o}(4,1)$ are treated separately in $[10,12]$ and $[6]$, and $\mathfrak{s o}(4,1)$ and $\mathfrak{s o}(5, \mathbb{R})$ are treated together in [18].)

The starting point is the complexification $\mathfrak{s o}(5, \mathbb{C})$, which is generated over $\mathbb{C}$ by $J_{ \pm}$and $K_{ \pm}$, the ladder operators on the order and degree. It is the complex span of $J_{ \pm}, K_{ \pm}, R_{ \pm}, S_{ \pm} ; J_{3}, K_{3}$, each of which is represented as in (9.4) and (9.6) by an infinite matrix indexed by $(\nu, \mu) \in\left(\nu_{0}, \mu_{0}\right)+\mathbb{Z}^{2}$. The elements $J_{3}, K_{3}$ span a Cartan subalgebra (an Abelian subalgebra of maximal [complex] dimension, here 2), which is represented diagonally:

$$
J_{3} \mathcal{S}_{v}^{\mu}=\mu \mathcal{S}_{v}^{\mu}, \quad K_{3} \mathcal{S}_{v}^{\mu}=\left(v+\frac{1}{2}\right) \mathcal{S}_{v}^{\mu} .
$$

When the representation of $\mathfrak{s o}(5, \mathbb{C})$ is reduced with respect to this subalgebra, it splits into an infinite direct sum of 1-dimensional representations, indexed by $(\nu, \mu)$. The corresponding real Cartan subalgebras of the $\mathfrak{s o}(3,2)$ and $\mathfrak{s o}(4,1)$ in Theorems 9.3 and 9.4(i) are the real spans of $\left\{J_{3}, K_{3}\right\}$ and $\left\{\mathrm{i} J_{3}, K_{3}\right\}$. For the $\mathfrak{s o}(3,2)$ and $\mathfrak{s o}(5, \mathbb{R})$ in Theorems 9.4(ii) and 9.5, they are the real span of $\left\{\mathrm{i} J_{3}, \mathrm{i} K_{3}\right\}$. (Recall that $D:=K_{3}$.) Only for the last two will the real Cartan subalgebra be represented by skew-Hermitian matrices, in fact, by imaginary diagonal ones.

It is readily verified that $J_{ \pm}, K_{ \pm}, R_{ \pm}, S_{ \pm} ; J_{3}, K_{3}$ can serve as a Cartan-Weyl basis of $\mathfrak{s o}(5, \mathbb{C})$, their complex span. That is, when the adjoint actions of $H_{1}:=J_{3}$ and $H_{2}:=K_{3}$ on this 10-dimensional Lie algebra are simultaneously diagonalized, the common eigenvectors ('root vectors') include $J_{ \pm}, K_{ \pm}, R_{ \pm}, S_{ \pm}$. The associated roots $\alpha \in \mathbb{R}^{2}$ are 2-tuples of eigenvalues, which can be identified with the displacements $\Delta(\nu, \mu)$, i.e., $\pm(0,1), \pm(1,0), \pm(1,1), \pm(1,-1)$. These form the $B_{2}$ root system. One can write

$$
\left[H_{i}, H_{j}\right]=0, \quad\left[H_{i}, E_{\alpha}\right]=\alpha_{i} E_{\alpha},
$$


where $E_{\alpha}$ is the root vector associated with root $\alpha$. The commutators $\left[E_{\alpha}, E_{\beta}\right]$ also prove to be consistent with the $B_{2}$ root system.

The Casimir invariants of $\mathfrak{s o}(5, \mathbb{C})$ and its three real forms can be computed from the commutation relations of the Cartan-Weyl basis elements. (For instance, the Killing form for the algebra yields a quadratic Casimir.) But it is easier to express them using the tensor operator $\mathbf{M}_{a b}$ of any of Theorems 9.3, 9.4, and 9.5. As elements of the universal enveloping algebra, the two Casimirs, quadratic and quartic, are defined thus $[10,12]$ :

$$
\begin{aligned}
& c_{2}:=-\frac{1}{2} \mathbf{M}_{a b} \mathbf{M}^{a b}, \\
& c_{4}:=-w_{a} w^{a},
\end{aligned}
$$

where $w^{a}=\frac{1}{8} \epsilon^{a b c d e} \mathbf{M}_{b c} \mathbf{M}_{d e}$ and the summation convention is employed, indices being raised and lowered by the tensors $\Gamma^{-1}=\left(g^{a b}\right)$ and $\Gamma=\left(g_{a b}\right)$. The Levi-Cività tensor $\epsilon_{a b c d e}$ is skew-symmetric in all indices, with $\epsilon_{12345}=+1$. The normalization and sign conventions are somewhat arbitrary.

Theorem 9.6 In the representation of the universal enveloping algebra of any of the real Lie algebras $\mathfrak{s o}(3,2), \mathfrak{s o}(4,1)$, and $\mathfrak{s o}(5, \mathbb{R})$ on the span of the generalized solid harmonics $\mathcal{S}_{\nu_{0}+n}^{\mu_{0}+m}(r, \theta, \varphi),(n, m) \in \mathbb{Z}^{2}$, the Casimirs $c_{2}$ and $c_{4}$ are represented by the constants $-\frac{5}{4}$ and 0 , irrespective of $\nu_{0}, \mu_{0}$.

Proof The proof is accomplished by the expressions for $\mathbf{M}_{a b}, \Gamma$ given in any of Theorems 9.3, 9.4, and 9.5, which imply that

$$
\begin{aligned}
c_{2} & =J \cdot J-P C^{+} \cdot P C^{+}+P C^{-} \cdot P C^{-}+D^{2} \\
& =J_{3}^{2}+K_{3}^{2}+\frac{1}{2}\left\{J_{+}, J_{-}\right\}-\frac{1}{2}\left\{K_{+}, K_{-}\right\}-\frac{1}{4}\left\{R_{+}, R_{-}\right\}-\frac{1}{4}\left\{S_{+}, S_{-}\right\},
\end{aligned}
$$

where $\{\cdot, \cdot\}$ is the anti-commutator. This expresses $c_{2}$ in terms of $J_{3}, K_{3}$ and the root vectors. The formula (9.16b) can be viewed as subsuming $J_{3}^{2}+\frac{1}{2}\left\{J_{+}, J_{-}\right\}$, which is the Casimir of the $\mathfrak{s o}(3, \mathbb{R})$ subalgebra spanned by $\left\{J_{+}, J_{-}, J_{3}\right\}$, and $K_{3}^{2}-\frac{1}{2}\left\{K_{+}, K_{-}\right\}$, which is the Casimir of the $\mathfrak{s o}(2,1)$ subalgebra spanned by $\left\{K_{+}, K_{-}, K_{3}\right\}$, and also, the Casimirs of the remaining two $\mathfrak{s o}(2,1)$ subalgebras. From the representations (9.4), (9.6) of $J_{ \pm}, K_{ \pm}, R_{ \pm}, S_{ \pm}$and $J_{3}, K_{3}$ as infinite matrices, one calculates from (9.16b) that $c_{2}$ (like $J_{3}, K_{3}$ ) is diagonal in $(n, m)$, with each diagonal element equaling $-\frac{5}{4}$.

For $\mathfrak{s o}(5, \mathbb{R})$, which is representative, the five components of $w^{a}$ include (i) the scalar i $J \cdot P C^{+}$, (ii) the three components of the vector $-\mathrm{i} P C^{-} \times P C^{+}+D J$, and (iii) the scalar $J \cdot P C^{-}$. These expressions, involving the scalar and vector product of three-vectors, must be interpreted with care: any product $A B$ of two Lie algebra elements signifies the symmetrized product $\frac{1}{2}\{A, B\}$. But by direct computation, one finds from (9.7), (9.8) and the infinite matrix representations (9.4), (9.6) that each component of $w^{a}$ is represented by the zero matrix, even (surprisingly) without symmetrization. 
This result is plausible, if not expected. In any unitary representation of a semisimple Lie group $G$ on $L^{2}(S), S$ being a homogeneous space $G / K$ of rank 1 , all Casimir operators except the quadratic one must vanish. (See [3] and [15, Chap. X].) Admittedly, the present representations of $\mathfrak{s o}(4,1)$, by real differential operators acting on multi-valued, non-square-integrable functions, are non-skew-Hermitian, and cannot be exponentiated to unitary representations of $S_{0}(4,1)$ of this 'most degenerate' type. The value $-\frac{5}{4}$ computed for the quadratic Casimir $c_{2}$, irrespective of $\left(v_{0}, \mu_{0}\right)$, can be viewed as the value of $j(j+1)$, where $j$ is a formal 'angular momentum' parameter equal to $-\frac{1}{2} \pm \mathrm{i}$.

For each $\left(v_{0}, \mu_{0}\right)$, the resulting representation of the real Lie algebra $\mathfrak{g}=\mathfrak{s o}(3,2)$, $\mathfrak{s o}(4,1)$ or $\mathfrak{s o}(5, \mathbb{R})$, or its universal enveloping algebra $\mathfrak{U}(\mathfrak{g})$, on the span of the generalized solid harmonics $\mathcal{S}_{\mathcal{v}_{0}+n}^{\mu_{0}+m},(n, m) \in \mathbb{Z}^{2}$, can be viewed linear-algebraically: as a homomorphism $\rho$ of real vector spaces, taking $\mathfrak{g}($ or $\mathfrak{U}(\mathfrak{g}))$ into the space of infinite matrices indexed by $(n, m)$. For each basis element $A \in \mathfrak{g}, \rho(A)$ is determined by (9.4), (9.6), and because the basis elements given in Theorems 9.4 and 9.5 include 'i' factors, the matrix elements of $\rho(A)$ may be complex.

To show that certain of these representations are substantially the same as known ones by infinite matrices that are skew-Hermitian, consider the effect of replacing the family $\left\{\mathcal{S}_{\nu}^{\mu}=\mathcal{S}_{\nu_{0}+n}^{\mu_{0}+m}\right\}$ by $\left\{\hat{\mathcal{S}}_{v}^{\mu}=\hat{\mathcal{S}}_{\nu_{0}+n}^{\mu_{0}+m}\right\}$, where the latter are 'twisted' by a square-root factor:

$$
\hat{\mathcal{S}}_{v}^{\mu}=\hat{\mathcal{S}}_{v}^{\mu}(r, \theta, \varphi):=\sqrt{\frac{\Gamma(v-\mu+1)}{\Gamma(v+\mu+1)}} r^{v} \mathrm{P}_{v}^{\mu}(\cos \theta) \mathrm{e}^{\mathrm{i} \mu \varphi} .
$$

That is, $\hat{\mathcal{S}}_{v}^{\mu}=r^{v} Y_{v}^{\mu}$, where

$$
Y_{v}^{\mu}(\theta, \varphi)=\sqrt{\frac{\Gamma(v-\mu+1)}{\Gamma(\nu+\mu+1)}} \mathrm{P}_{\nu}^{\mu}(\cos \theta) \mathrm{e}^{\mathrm{i} \mu \varphi} .
$$

When $v=0,1,2, \ldots$, with $\mu=-v, \ldots, v$, this $Y_{v}^{\mu}$ is the classical (complex) spherical harmonic on $S^{2}$, of degree $v$ and order $\mu .^{2}$ In this case, the square root factor equals $\left[(v+\mu+1)_{2 \mu}\right]^{-1 / 2}$ and is positive by convention; a discussion of how to interpret it in other cases is deferred. At least formally, the representation of $\mathfrak{g}$ or $\mathfrak{U}(\mathfrak{g})$ on $\left\{\hat{\mathcal{S}}_{\nu_{0}+n}^{\mu_{0}+m}\right\}$ comes from that on $\left\{\mathcal{S}_{\nu_{0}+n}^{\mu_{0}+m}\right\}$ by a diagonal similarity transformation. The formulas

$$
\begin{aligned}
& J_{ \pm} \hat{\mathcal{S}}_{v}^{\mu}=\sqrt{\left(v-\mu+\frac{1}{2} \mp \frac{1}{2}\right)\left(v+\mu+\frac{1}{2} \pm \frac{1}{2}\right)} \hat{\mathcal{S}}_{v}^{\mu \pm 1} \\
& K_{ \pm} \hat{\mathcal{S}}_{v}^{\mu}=\sqrt{\left(v-\mu+\frac{1}{2} \pm \frac{1}{2}\right)\left(v+\mu+\frac{1}{2} \pm \frac{1}{2}\right)} \hat{\mathcal{S}}_{v \pm 1}^{\mu}, \\
& R_{ \pm} \hat{\mathcal{S}}_{v}^{\mu}=\sqrt{\left(v+\mu+\frac{1}{2} \pm \frac{1}{2}\right)\left(v+\mu+\frac{1}{2} \pm \frac{3}{2}\right)} \hat{\mathcal{S}}_{v \pm 1}^{\mu \pm 1}, \\
& S_{ \pm} \hat{\mathcal{S}}_{v}^{\mu}=\sqrt{\left(v-\mu+\frac{1}{2} \pm \frac{1}{2}\right)\left(v-\mu+\frac{1}{2} \pm \frac{3}{2}\right)} \hat{\mathcal{S}}_{v \pm 1}^{\mu \mp 1}
\end{aligned}
$$

\footnotetext{
2 The orthonormalization factor $\sqrt{(2 v+1) / 4 \pi}$, appropriate for an inner product on $S^{2}$, is omitted. But this $Y_{v}^{\mu}$ automatically includes the so-called Condon-Shortley factor, owing to the definition of $\mathrm{P}_{v}^{\mu}$ used here (see Sect. 2).
} 
and

$$
J_{3} \hat{\mathcal{S}}_{v}^{\mu}=\mu \hat{\mathcal{S}}_{v}^{\mu}, \quad K_{3} \hat{\mathcal{S}}_{v}^{\mu}=\left(v+\frac{1}{2}\right) \hat{\mathcal{S}}_{v}^{\mu}
$$

now replace (9.4) and (9.6), but the actions of the elements $J_{i}, K_{i}, P C_{i}^{ \pm}, D$ are still defined in terms of these by (9.14) and (9.15).

Theorem 9.7 If $\left(v_{0}, \mu_{0}\right)=(0,0)$ or $\left(\frac{1}{2}, \frac{1}{2}\right)$, the representation $\rho$ of $\mathfrak{g}=\mathfrak{s o}(3,2)$ on the span of $\left\{\hat{\mathcal{S}}_{v}^{\mu}=\hat{\mathcal{S}}_{v_{0}+n}^{\mu_{0}+m}\right\},(n, m) \in \mathbb{Z}^{2}$, which is obtained from (9.19),(9.20) by identifying $\mathfrak{s o}(3,2)$ with the real span of $\mathrm{i} J_{1}, \mathrm{i} J_{2}, \mathrm{i} J_{3} ; \mathrm{i} P C_{1}^{+}, \mathrm{i} P C_{2}^{+}, \mathrm{i} P C_{3}^{+}$; $P C_{1}^{-}, P C_{2}^{-}, P C_{3}^{-} ; \mathrm{i} D$ as in Theorem 9.4(ii), has an irreducible constituent that is defined on the subspace spanned by $\left\{\hat{\mathcal{S}}_{\nu}^{\mu}=\hat{\mathcal{S}}_{\nu_{0}+n}^{\mu_{0}+m}\right\}$ with $n=0,1,2, \ldots$ and $\mu=-v,-v+1 \ldots, v-1, v$. On this subspace, every element of $\mathfrak{g}$ is represented by an infinite matrix that is skew-Hermitian.

Proof By (9.19), if $\mu=v$ then $J_{+}, K_{-}, S_{-}$give zero when acting on $\hat{\mathcal{S}}_{v}^{\mu}$, and if $\mu=-v$ then $J_{-}, K_{-}, R_{-}$give zero. Thus $\rho$ is reducible: it can be restricted to the stated subspace. On this subspace, the formal similarity transformation performed by the square root factor in (9.17) is not singular: only if $v \pm \mu$ is a negative integer will one of the gammas be infinite. For $\left(v_{0}, \mu_{0}\right)$ equal to either of $(0,0)$ and $\left(\frac{1}{2}, \frac{1}{2}\right)$, the square root factor simply equals $\left[(\nu+\mu+1)_{2 \mu}\right]^{-1 / 2}$.

By (9.19) and (9.20), each of $\rho\left(J_{ \pm}\right), \rho\left(K_{ \pm}\right), \rho\left(R_{ \pm}\right), \rho\left(S_{ \pm}\right) ; \rho\left(J_{3}\right), \rho\left(K_{3}\right)$ is a real matrix, the plus and minus versions being transposes of each other, and $\rho\left(J_{3}\right), \rho\left(K_{3}\right)$ being symmetric (and diagonal). It follows from (9.14) and (9.15) that the $\rho\left(J_{i}\right)$, the $\rho\left(P C_{i}^{+}\right)$, and $\rho(D)$ are Hermitian, and the $\rho\left(P C_{i}^{-}\right)$are skew-Hermitian. The claim follows.

Being skew-Hermitian, the two infinite-dimensional representations of $\mathfrak{s o}(3,2)$ in Theorem 9.7 exponentiate to (irreducible) unitary representations of the so-called antide Sitter group $S O_{0}(3,2)$, or its universal cover. The latter have been classified [10,12], and the ones arising from the theorem can be identified. They are the remarkable Dirac singleton representations, with whimsical names [7,14]: the $\left(v_{0}, \mu_{0}\right)=\left(\frac{1}{2}, \frac{1}{2}\right)$ one is 'Di' and the $\left(v_{0}, \mu_{0}\right)=(0,0)$ one is 'Rac.' For the Dirac singletons, the Casimirs $\left(c_{2}, c_{4}\right)$ have long been known to equal $\left(-\frac{5}{4}, 0\right)$. (See [2, Sect. III].) They are singleton representations in the sense that if they are reduced with respect to the subalgebra $\mathfrak{g}_{0}=\mathfrak{s o}(3, \mathbb{R}) \oplus_{\mathbb{R}} \mathfrak{s o}(2, \mathbb{R})$, thereby being split into representations of $\mathfrak{g}_{0}$, each of the latter that appears, does so with unit multiplicity. The ones that appear are labeled uniquely by $v=v_{0}+n, n=0,1,2, \ldots$.

The Rac representation of $\mathfrak{s o}(3,2)$ is realized by differential operators on $\mathbb{R}^{3}$ (with complex coefficients), expressions for which follow immediately from (9.13). They act on the span of the classical solid harmonics, $\left\{\hat{\mathcal{S}}_{n}^{m}\right\}$ with $n=0,1,2, \ldots$ and $m=-n, \ldots, n$. Kyriakopoulos [19] in effect discovered that the Rac has such a realization, before the name was coined, and extended this result to higher dimensions. The Di is realized by the same operators, acting on the span of the 'spinorial' solid harmonics $\left\{\hat{\mathcal{S}}_{n+\frac{1}{2}}^{m}\right\}$ with $n=0,1,2, \ldots$ and $m=-n-\frac{1}{2}, \ldots, n+\frac{1}{2}$. The existence of this realization seems not to be known. This is perhaps because the solid harmonics of half-odd-integer degree and order are double-valued on $\mathbb{R}^{3}$, are typically 
non-square-integrable, and are based on the little-known dihedral Ferrers functions. The expressions for the dihedral Ferrers functions in terms of Jacobi polynomials, given in Theorem 8.5 above, are new.

The representations of $\mathfrak{s o}(3,2)$ carried by the octahedral and tetrahedral families of solid harmonics, $\left\{\mathcal{S}_{v}^{\mu}=\mathcal{S}_{v_{0}+n}^{\mu_{0}+m}\right\}$ with $\left(v_{0}+\frac{1}{2}, \mu_{0}\right)$ equal to $\left( \pm \frac{1}{3}, \pm \frac{1}{4}\right),\left( \pm \frac{1}{4}, \pm \frac{1}{3}\right)$, and $\left( \pm \frac{1}{3}, \pm \frac{1}{3}\right)$, are not skew-Hermitian, even up to diagonal equivalence. Twisting the basis to $\left\{\hat{\mathcal{S}}_{v}^{\mu}=\hat{\mathcal{S}}_{v_{0}+n}^{\mu_{0}+m}\right\}$ does not help matters, because only if $\nu_{0}, \mu_{0}$ are both integers or both half-odd-integers, which without loss of generality may be taken to be 0,0 or $\frac{1}{2}, \frac{1}{2}$, does it permit the representation to be restricted to a subspace spanned by the harmonics with $v=v_{0}+n, n=0,1,2, \ldots$, and $\mu=-v,-v+1 \ldots, v-1, v$.

For general $\left(v_{0}, \mu_{0}\right)$, there is accordingly no restriction on the index $(n, m) \in \mathbb{Z}^{2}$ of the basis functions of the representation, and the square roots in (9.17), (9.18), (9.19) may be square roots of negative quantities. Irrespective of what sign convention for the square root is adopted, the resulting imaginary factors will interfere with skewHermiticity, and upon integration of the representation, with unitarity. In fact, the familiar definition of the (surface) spherical harmonic $Y_{v}^{\mu}=Y_{v}^{\mu}(\theta, \varphi)$ given in (9.18), incorporating the square root factor, seems to be useful only when the degree $v$ and the order $\mu$ are both integers or both half-odd-integers.

Open Access This article is distributed under the terms of the Creative Commons Attribution 4.0 International License (http://creativecommons.org/licenses/by/4.0/), which permits unrestricted use, distribution, and reproduction in any medium, provided you give appropriate credit to the original author(s) and the source, provide a link to the Creative Commons license, and indicate if changes were made.

\section{References}

1. Bogdanović, R., Whitehead, M.A.: The representation of the $S O(4,1)$ group in four-dimensional Euclidean and spinor space. J. Math. Phys. 16(2), 400-409 (1975)

2. Bohm, A., Loewe, M., Magnollay, P., Tarlini, M., Aldinger, R.R., Biedenharn, L.C., van Dam, H.: Quantum relativistic oscillator. III. Contraction between the algebras of $S O(3,2)$ and the three-dimensional harmonic oscillator. Phys. Rev. D 32(10), 2828-2834 (1985)

3. Boyer, C.P.: Matrix elements for the most degenerate continuous principal series of representations of $S O(p, 1)$. J. Math. Phys. 12(8), 1599-1603 (1971)

4. Celeghini, E., del Olmo, M.A.: Algebraic special functions and $S O(3,2)$. Ann. Phys. 333, 90-103 (2013). arXiv:1210.5192 [math-ph]

5. Detrich, J., Conn, R.W.: Finite sum evaluation of the Gauss hypergeometric function in an important special case. Math. Comput. 33, 788-791 (1979)

6. Dixmier, J.: Représentations intégrables du groupe de De Sitter. Bull. Soc. Math. Fr. 89, 9-41 (1961)

7. Dobrev, V.K., Sezgin, E.: Spectrum and character formulæ of so(3,2) unitary representations. In: Hennig, J.D., Lücke, W., Tolar, J. (eds.) Differential Geometry, Group Representations, and Quantization, no. 379 in Lecture Notes in Physics, pp. 227-238. Springer, New York (1991)

8. Dong, S.H.: Factorization Method in Quantum Mechanics. Springer, Dordrecht (2007)

9. Durand, L.: Fractional operators and special functions. II. Legendre functions. J. Math. Phys. 44(5), 2266-2292 (2003). arXiv:math-ph/0212019

10. Ehrman, J.B.: On the unitary irreducible representations of the universal covering group of the $3+2$ DeSitter group. Proc. Cambr. Philos. Soc. 53, 290-303 (1957)

11. Erdélyi, A., Magnus, W., Oberhettinger, F., Tricomi, F.G. (eds.): Higher Transcendental Functions. McGraw-Hill, New York (1953-55). Also known as The Bateman Manuscript Project

12. Evans, N.T.: Discrete series for the universal covering group of the $3+2$ de Sitter group. J. Math. Phys. 8(2), 170-184 (1967) 
13. Everitt, W.N.: A catalogue of Sturm-Liouville differential equations. In: Amrein, W.O., Hinz, A.M., Pearson, D.B. (eds.) Sturm-Liouville Theory, pp. 271-331. Birkhaüser, Boston (2005)

14. Flato, M., Frønsdal, C.: Dis and Racs. Phys. Lett. 97B(2), 236-240 (1980)

15. Helgason, S.: Differential Geometry and Symmetric Spaces. Academic Press, New York (1962)

16. Hobson, E.W.: The Theory of Spherical and Ellipsoidal Harmonics. Cambridge University Press, Cambridge (1931)

17. Ilk, K.H.: Ein Beitrag zur Dynamik ausgedehnter Körper: Gravitationswechselwirkung. Beck, Munich (1983)

18. Kuriyan, J.G., Mukunda, N., Sudarshan, E.C.G.: Master analytic representations and unified representation theory of certain orthogonal and pseudo-orthogonal groups. Commun. Math. Phys. 8, 204-227 (1968)

19. Kyriakopoulos, E.: Dynamical groups and the Bethe-Salpeter equation. Phys. Rev. 174(5), 1846-1859 (1968)

20. Love, E.R.: Abel summability of certain series of Legendre functions. Proc. Lond. Math. Soc. (3) 69(3), 629-672 (1994)

21. Love, E.R., Hunter, M.N.: Expansions in series of Legendre functions. Proc. Lond. Math. Soc. (3) 64(3), 579-601 (1992)

22. MacRobert, T.M.: The Mehler-Dirichlet integral and some other Legendre function formulæ. Philos. Mag. 7(14), 632-656 (1932)

23. Maier, R.S.: Legendre functions of fractional degree: transformations and evaluations. Proc. R. Soc. Lond. Ser. A 472(2188), 20160097, 29 pp. (2016). arXiv:1602.03070

24. Mason, J.C., Handscomb, D.C.: Chebyshev Polynomials. Chapman \& Hall/CRC, Boca Raton, FL (2003)

25. Matsuda, M.: Lectures on Algebraic Solutions of Hypergeometric Differential Equations. Kinokuniya Co., Tokyo (1985)

26. Miller Jr., W.: Lie theory and generalizations of the hypergeometric functions. SIAM J. Appl. Math. 25(2), 226-235 (1973)

27. Miller Jr., W.: Symmetries of differential equations. the hypergeometric and Euler-Darboux equations. SIAM J. Math. Anal. 4(2), 314-328 (1973)

28. Miller Jr., W.: Symmetry and Separation of Variables, The Encyclopedia of Mathematics and its Applications, vol. 4. Addison-Wesley, Reading, MA (1977)

29. Ochiai, H., Yoshida, M.: Polynomials associated with the hypergeometric functions with finite monodromy groups. Int. J. Math. 15(7), 629-649 (2004)

30. Olver, F.W.J.: Asymptotics and Special Functions. AKP Classics edn. A. K. Peters, Wellesley, MA (1997)

31. Olver, F.W.J., Lozier, D.W., Boisvert, R.F., Clark, C.W. (eds.): NIST Handbook of Mathematical Functions. U.S. Department of Commerce, National Institute of Standards and Technology, Washington, DC (2010)

32. Pinsky, M.A.: Endpoint convergence of Legendre series. In: Bray, W.O., Stanojević, Č. V. (eds.) Analysis of Divergence, Chap. 6, pp. 79-85. Birkhäuser, Boston (1999)

33. Poole, E.G.C.: Introduction to the Theory of Linear Differential Equations. Oxford University Press, Oxford (1936)

34. Ronveaux, A. (ed.): Heun's Differential Equations. Oxford University Press, Oxford (1995). (With contributions by F. M. Arscott, S. Yu. Slavyanov, D. Schmidt, G. Wolf, P. Maroni and A. Duval)

35. Schwarz, H.A.: Ueber diejenigen Fälle, in welchen die Gaussische hypergeometrische Reihe eine algebraische Function ihres vierten Elementes darstellt. J. Reine Angew. Math. 75, 292-335 (1873)

36. Truesdell, C.: An Essay Toward a Unified Theory of Special Functions. Princeton University Press, Princeton, NJ (1948)

37. Vidūnas, R.: Expressions for values of the gamma function. Kyushu J. Math. 59(2), 267-283 (2005). arXiv:math/0403510 [math.CA]

38. Vidūnas, R.: Dihedral Gauss hypergeometric functions. Kyushu J. Math. 65(1), 141-167 (2011). arXiv:0807.4888 [math.CA]

39. Whittaker, E.T., Watson, G.N.: A Course of Modern Analysis, 4th edn. Cambridge University Press, Cambridge (1927) 